\title{
A Low-cost Normalized Difference Vegetation Index (NDVI) Payload for Cubesats and Unmanned Aerial Vehicles (UAVs)
}

\author{
Steven Hard
}

Follow this and additional works at: https://researchrepository.wvu.edu/etd

\section{Recommended Citation}

Hard, Steven, "A Low-cost Normalized Difference Vegetation Index (NDVI) Payload for Cubesats and Unmanned Aerial Vehicles (UAVs)" (2018). Graduate Theses, Dissertations, and Problem Reports. 5761. https://researchrepository.wvu.edu/etd/5761

This Thesis is protected by copyright and/or related rights. It has been brought to you by the The Research Repository @ WVU with permission from the rights-holder(s). You are free to use this Thesis in any way that is permitted by the copyright and related rights legislation that applies to your use. For other uses you must obtain permission from the rights-holder(s) directly, unless additional rights are indicated by a Creative Commons license in the record and/ or on the work itself. This Thesis has been accepted for inclusion in WVU Graduate Theses, Dissertations, and Problem Reports collection by an authorized administrator of The Research Repository @ WVU. For more information, please contact researchrepository@mail.wvu.edu. 


\title{
A Low-Cost Normalized Difference Vegetation Index (NDVI) Payload for Cubesats and Unmanned Aerial Vehicles (UAVs)
}

\author{
Steven Hard, BSME, BSAE, MSAE
}

\author{
Thesis Submitted to the Statler College of Engineering \\ and Mineral Resources at West Virginia University in \\ Partial Fulfillment of the Requirements for the Degree of \\ Masters of Science \\ in \\ Electrical Engineering \\ Powsiri Klinkhachorn, Ph.D., Chair \\ Roy Nutter, Ph. D. \\ Mario Perhinschi, Ph.D.
}

Lane Department of Computer Science and Electrical Engineering

\section{Morgantown, West Virginia}

2018

Keywords: NDVI, vegetation, analysis, pixel, earth science, atmosphere, solar altitude, incidence Copyright 2018 Steven Hard 


\section{Abstract \\ A Low-Cost Normalized Difference Vegetation Index (NDVI) Payload for Cubesats and Unmanned Aerial Vehicles (UAVs)}

\section{Steven Hard}

The focus of this research has been the design and fabrication of a Normalized Difference Vegetation Index (NDVI) payload configuration. This unique payload employs low-cost commercial off-the-shelf (COTS) hardware and equipment to assess photosynthetic activity and vegetation health through remote sensing on Cubesat or Unmanned Aerial Vehicle (UAV) platforms. The proposed NDVI imaging payload is comprised of three main subsystems: an electrical system, a software system, and a hardware system. The electrical system includes a custom designed printed circuit board (PCB), a single cell 3.7 V lithiumpolymer battery, voltage regulator circuitry components, and wiring harnesses and connectors. The software system employs a master and slave system that communicates through general purpose input/output (GPIO) pin responses. Raspberry Pi Zero computer boards serve as the central processing units (CPUs) of the hardware subsystem, which also consists of the Pi-Cam standard red/green/blue (RGB) and Pi No-IR near-infrared (NIR) camera modules. A PCB was designed to be compatible with the Cubesat standard and lightweight component selections make it a desirable option for UAV flights. Open-source GIMP image processing software was used to analyze results from ground-based testing and flight testing on a UAV and general aviation (GA) aircraft at various altitudes to validate proof of concept. Raw NDVI and NDVI color map images were created from GIMP postprocessing. Analysis of the results suggests that the angle of incidence of the sun with respect to the view angle of the imaging payload is a significant factor in the resulting NDVI values. Terrain also appeared to have an effect on the results where shadows were cast from the sun at low angles of incidence. Therefore, in the northern hemisphere it is recommended that image collection is performed roughly within the hours of 10 AM and 2 PM between the vernal and autumnal equinoxes to ensure a solar altitude of at least $35^{\circ}$. For best results, it has been verified that image data should be collected at the local time of maximum solar altitude for a particular date and location of interest (typically around noon). The information gather by this research can be used by scientists and technologist to potentially provide a means of enhancing their research and further developing technologies of UAV applications and space-based systems. 


\section{Table of Contents}

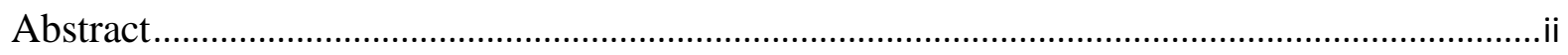

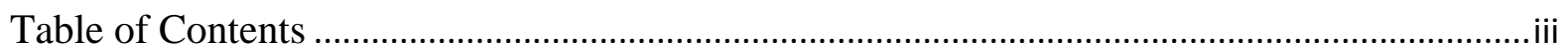

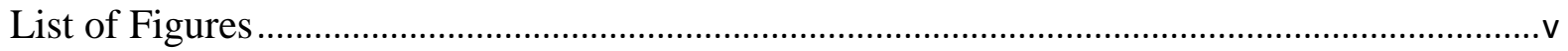

List of Tables ................................................................................................................... vii

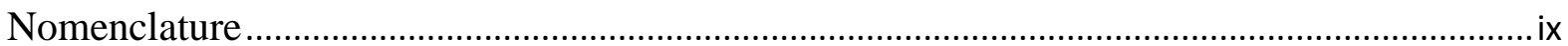

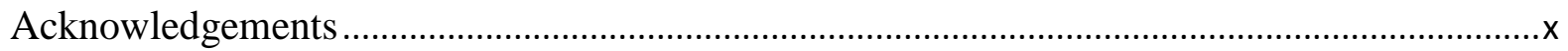

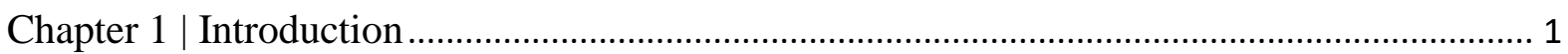

$1.1 \quad$ Normalized Difference Vegetation Index ……..................................................... 1

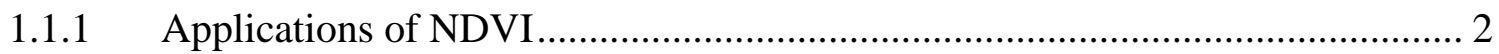

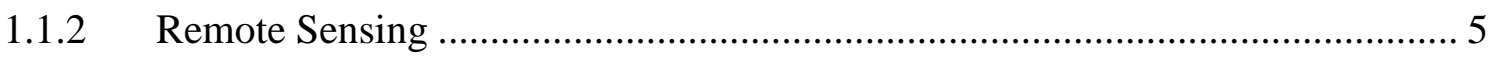

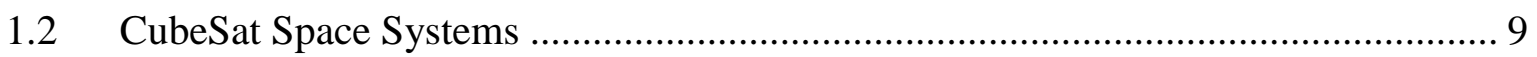

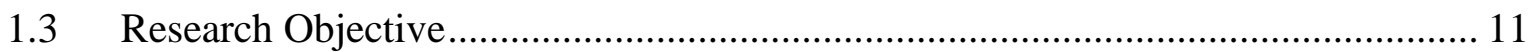

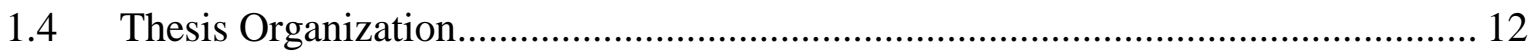

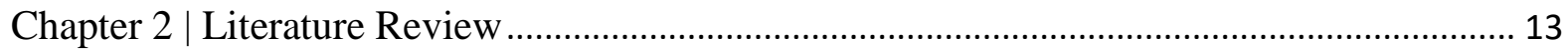

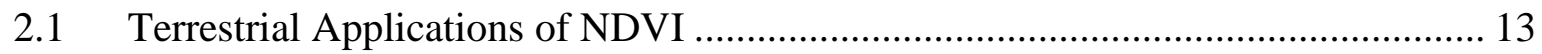

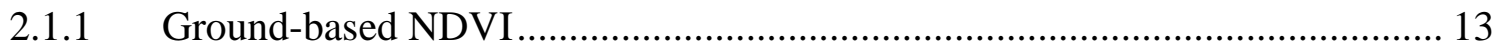

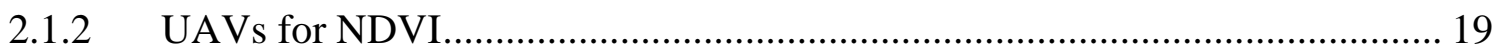

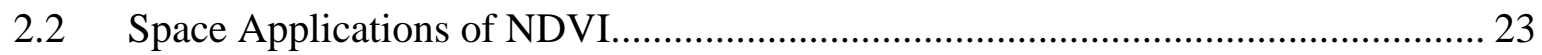

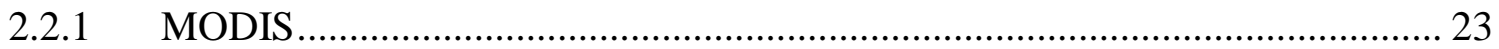

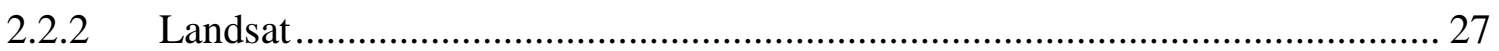

2.3 Cubesats for Earth Science............................................................................... 30

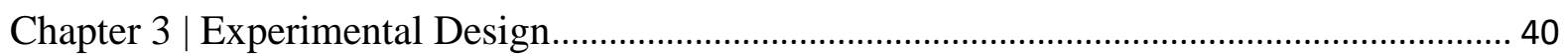

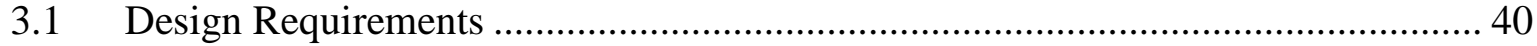

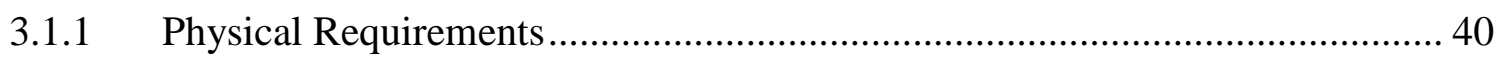

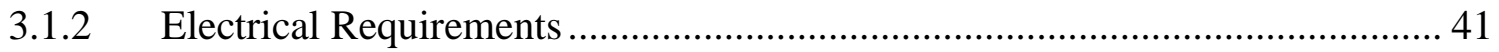

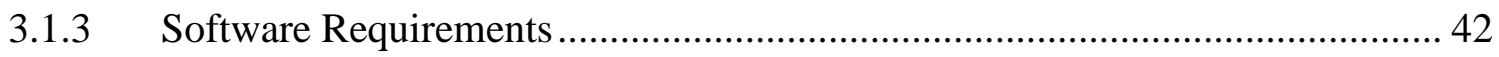

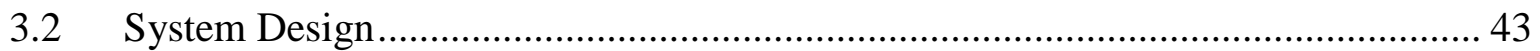

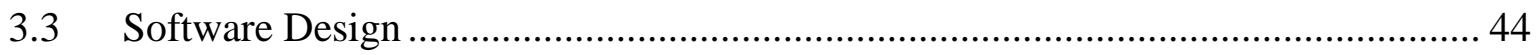

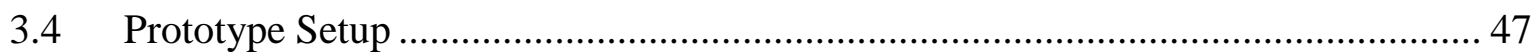

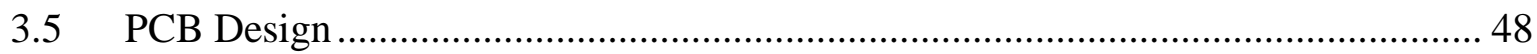

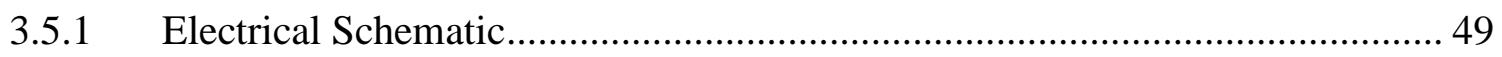

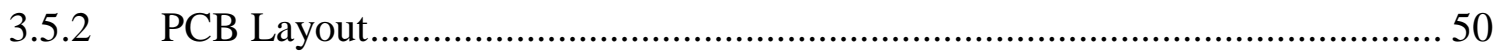

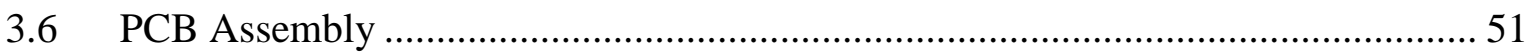

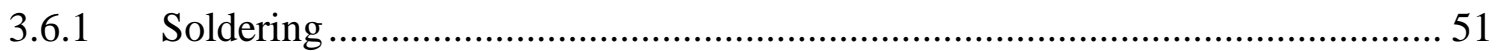

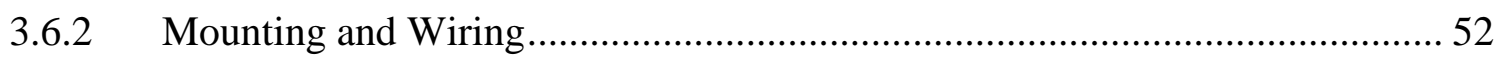

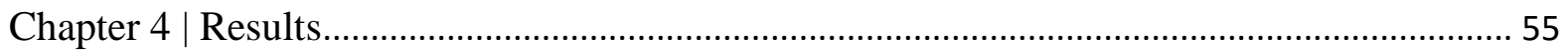

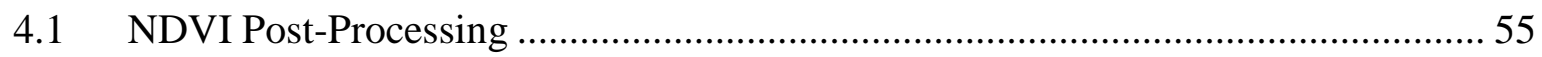




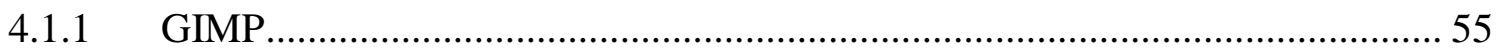

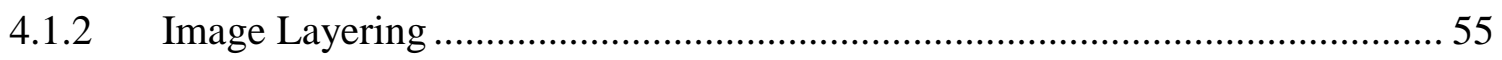

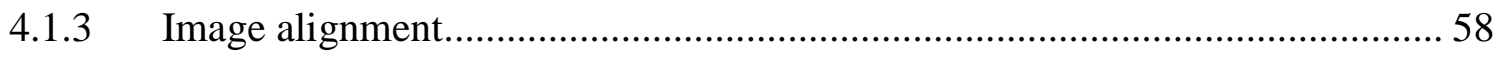

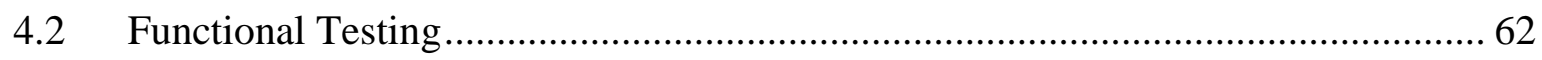

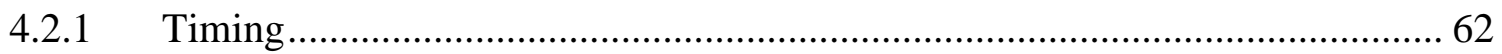

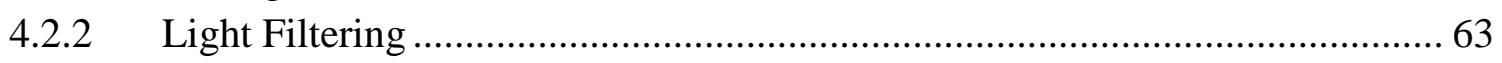

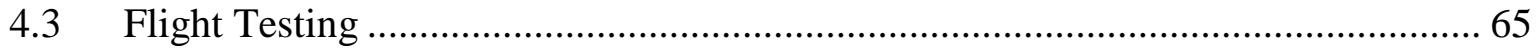

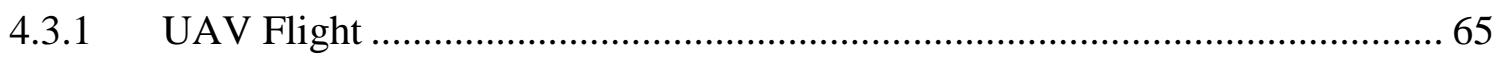

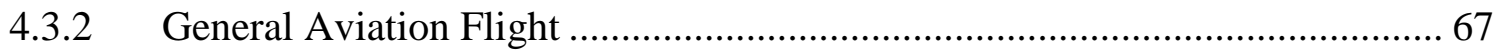

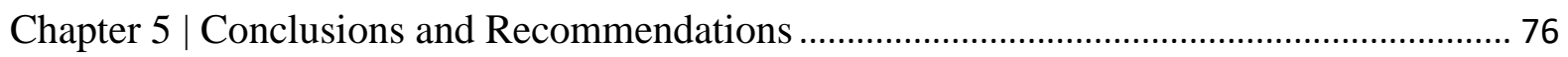

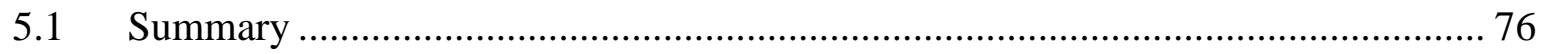

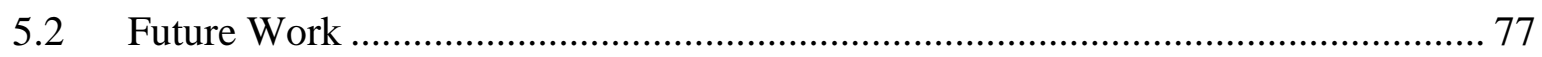

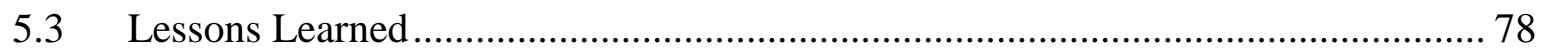

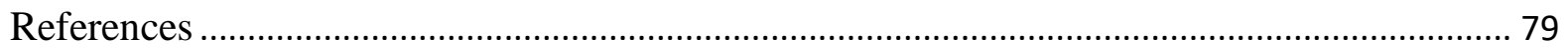

Appendix A: Preliminary Assessment of Cubesat Utility ..................................................... 82

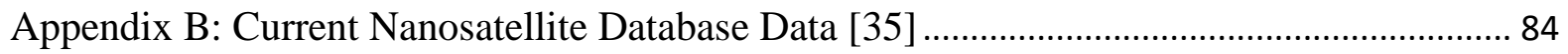

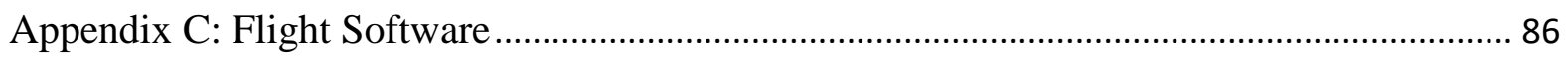

Appendix D: Electronic Parts List ................................................................................. 90 


\section{List of Figures}

Figure 1: Comparison of Reflected Visible and Near-Infrared Light for Green and

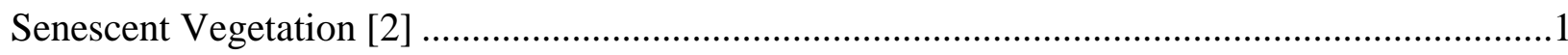

Figure 2: NDVI Anomaly in the U.S. for August 1993 [7] ................................................. 3

Figure 3: The Principle of Hysteresis [12] .......................................................................... 5

Figure 4: SIERRA $($ Wingspan $=6 \mathrm{~m})$ on the Tarmac at ................................................. 6

Figure 5: Helium Blimp $($ Fuselage $=4 \mathrm{~m})$ with Camera Rig in Flight [19] ................................ 7

Figure 6: Flight Testing of Quadcopter UAV (Model MD4-1000) over.................................... 7

Figure 7: Sample of the MODIS/Terra 16-day NDVI ...................................................... 8

Figure 8: Effects of Drought Captured by the LandSat-7 Satellite [25] ................................... 9

Figure 9: Simplified Subset of a Typical Organization Chart for a .......................................... 10

Figure 10: Longitudinal Cross-section of NDVI Hand-held Device (55 mm length, 43

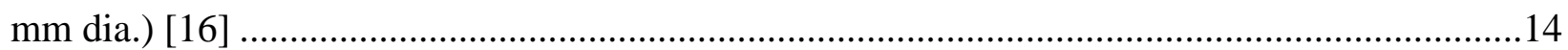

Figure 11: Solar Transmittance vs Ratio of Global Radiation at Ground Level ( $\mathrm{Rg}$ ) to Top of Atmosphere (Rexo) in 2019 for Five Levels of Sunshine (1-overcast, 5-clear sky) [17] .........15

Figure 12: Ground-based NDVI Time-series over Congo African Savanna [17] ..................... 16

Figure 13: Ground-based NDVI Time-series over a Succession of Crops in Belgium (2007-Winter Wheat, 2008-Sugar Beet, 2009-Winter Wheat and Mustard) [17] .......................17

Figure 14: 'Sensicle' Ground Vehicle Platform with Various Sensors Integrated [18] .............. 19

Figure 15: Typical Helium Blimp with Camera Payload in Flight [19] ..................................... 22

Figure 16: NASA Earth Science Division Operating Missions [30] ....................................... 23

Figure 17: 2000-2006 Cumulative Change in Growing Season NDVI for the Western United States ..................................................................................................................26

Figure 18: Asian Grassland Study Area with Land-use/Land-cover Types and Locations

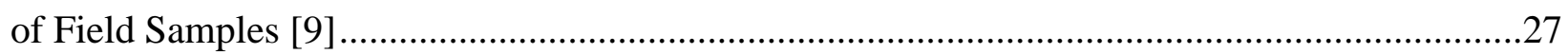

Figure 19: NDVI and NDWI Relationships for Corn and Soybean ......................................... 29

Figure 20: Image Segmentation Partition of Image into Objects (a) Raw Satellite Image with 800x1000 Pixels (b) Application of QT and MT Segmentation [33] ................................ 30

Figure 21:Standard Cubesat Platform [26] ........................................................................ 32

Figure 22: Cubesat Mission Breakdown by Mission Category, 2000-2012 [36] ....................... 34

Figure 23: Number of Cubesats Launched Per Year and Per Launch Vehicle from 2000-

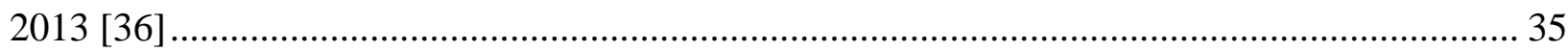

Figure 24: Fully-Assembled STF-1 Cubesat [40]............................................................. 36

Figure 25: 3D Mechanical Model of the OCF-1 Payload Board ............................................ 37

Figure 26: 3D Printed Model Next to Stacked Assembly of IOS TubeSat Kit (left) and a Fully Assembled Tubesat (right) .................................................................................... 38

Figure 27: 1.3U IOS Cubesat Kit (left) and 1U IOS CubeSat Kit Inside Aluminum

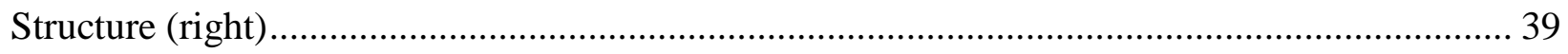

Figure 28: Design Dimensions of the Payload PCB ......................................................... 40 
Figure 29: LM2731 Basic Application Circuit [45] .......................................................... 41

Figure 30: Function Block Diagram of the Experimental NDVI Payload................................ 44

Figure 31: Master CPU Software Flowchart ...................................................................... 46

Figure 32: Slave CPU Software Flowchart.................................................................... 47

Figure 33: Portable NDVI Prototype Platform Taking an Image ............................................. 48

Figure 34: Voltage Regulation Circuit Schematic .................................................................... 49

Figure 35: Raspberry Pi Header Pinout Schematic.............................................................. 50

Figure 36: Adafruit 10DOF IMU Header Pinout Schematic ................................................... 50

Figure 37: PCB Design of the NDVI CubeSat/UAV Payload .............................................. 51

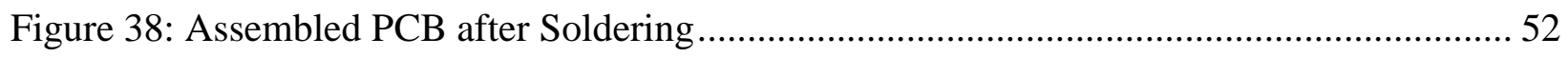

Figure 39: Top View Photo of Payload Assembly .................................................................. 53

Figure 40: Side View Photo of Payload Assembly.................................................................. 53

Figure 41: Bottom View Photo of Payload Assembly.......................................................... 54

Figure 42: GIMP Image Layer Panels Illustrating NDVI Post-Processing Procedure (top

left- Addition, top right-Subtract, bottom left-Divide, bottom right-Merge) ............................ 57

Figure 43: Original RGB (left) and NIR (right) Images Captured during Field Testing............. 58

Figure 44: Raw NDVI and NDVI Gradient Color Map Results from Field Testing.................. 58

Figure 45: Close-up RGB (left) and NIR (right) Images Showing Slight Offset ...................... 59

Figure 46: NIR Image with 50\% Transparency Showing RGB Image Offset ......................... 60

Figure 47: NIR Image with 50\% Transparency Showing RGB Image Alignment .................... 60

Figure 48: Cropped RGB (left) and NIR (right) Images of Close-up Still Shot........................ 60

Figure 49: Raw NDVI (left) and NDVI Color Map (right) of Close-up Still Shot ................... 61

Figure 50: Original RGB (left) and NIR (right) Images of Landscape Still Shot...................... 61

Figure 51: Raw NDVI (left) and NDVI Color Map (right) of Landscape Still Shot.................. 62

Figure 52: Timing Sequence of the RGB Camera (left) and NIR Camera (right) Image

Capture on the First Iteration (top) and the Second Iteration (bottom) .................................... 63

Figure 53: Original RGB (left) and NIR (right) Images of Landscape Still Shot with Blue

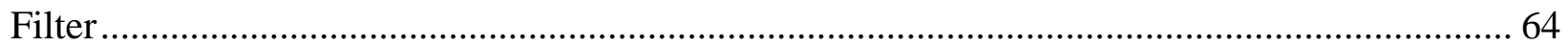

Figure 54: Raw NDVI (left) and NDVI Color Map (right) of Landscape Still Shot with

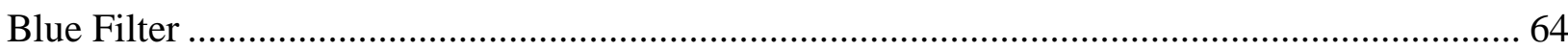

Figure 55: Original RGB (left) and NIR (right) Images of Landscape Still Shot with

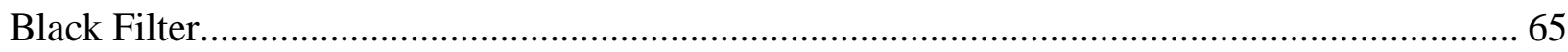

Figure 56: Raw NDVI (left) and NDVI Color Map (right) of Landscape Still Shot with

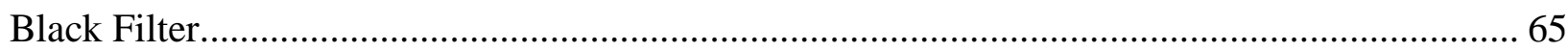

Figure 57:|NDVI Payload Attached to the Parrot AR.Drone Quadcopter................................. 66

Figure 58: Original RGB (left) and NIR (right) Images of Grassy Area Taken with UAV

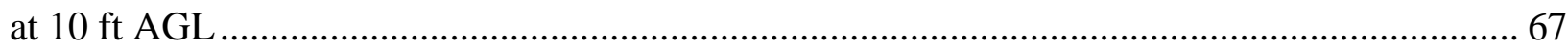

Figure 59: Raw NDVI (left) and NDVI Color Map (right) of Grassy Area Taken with

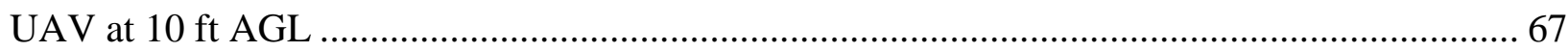

Figure 60: RGB Photos Taken during Takeoff in a Cessna 152 General Aviation Airplane ....... 68 
Figure 61: Original RGB (left) and NIR (right) Images of Woodline and Field Taken with

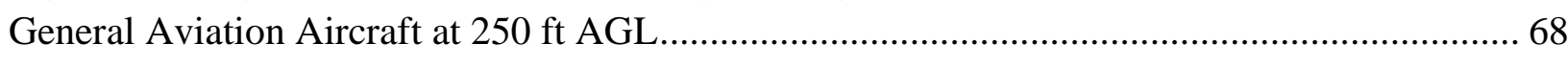

Figure 62: Raw NDVI (left) and NDVI Color Map (right) of Woodline and Field Taken

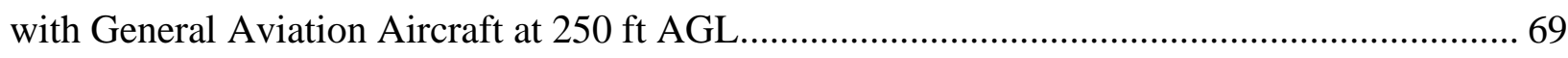

Figure 63: Original RGB (left) and NIR (right) Images of Ridgeline Treetops with

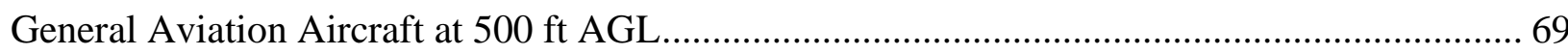

Figure 64: Raw NDVI (left) and NDVI Color Map (right) of Ridgeline Treetops with

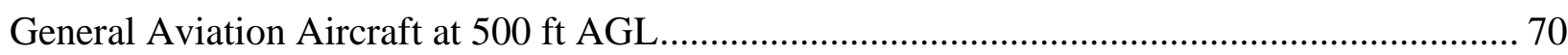

Figure 65: Original RGB (left) and NIR (right) Images of Ridgeline Treetops with

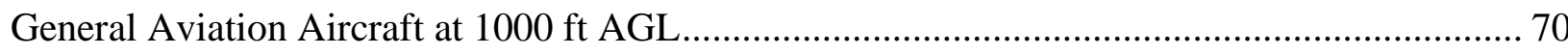

Figure 66: Raw NDVI (left) and NDVI Color Map (right) of Ridgeline Treetops with

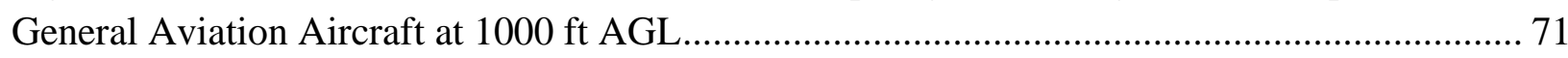

Figure 68: Original RGB (left) and NIR (right) Images of Rock Quarry with General Aviation Aircraft at $2000 \mathrm{ft}$ AGL ………………………...................................................... 71

Figure 69: Raw NDVI (left) and NDVI Color Map (right) of Rock Quarry with General Aviation Aircraft at $2000 \mathrm{ft}$ AGL 72

Figure 70: Original RGB (left) and NIR (right) Images of Ridgeline and Field with

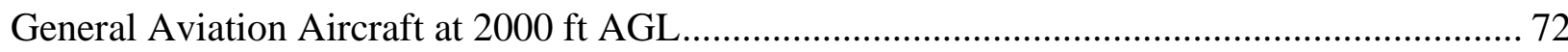

Figure 71: Raw NDVI (left) and NDVI Color Map (right) of Ridgeline and Field with General Aviation Aircraft at $2000 \mathrm{ft}$ AGL.............................................................................. 73

Figure 71: Original RGB (left) and NIR (right) Images of Cheat Lake with General

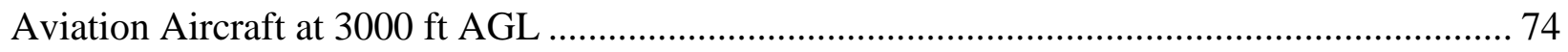

Figure 72: Raw NDVI (left) and NDVI Color Map (right) of Cheat Lake with General

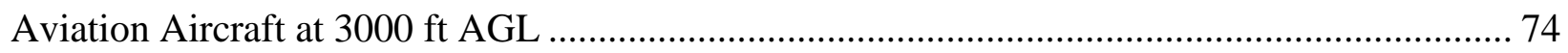

Figure 73: Original RGB (left) and NIR (right) Images of Ridgeline with General

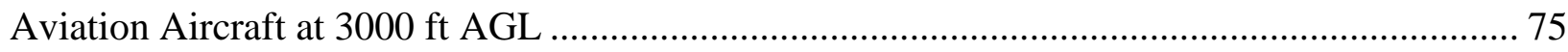

Figure 74: Raw NDVI (left) and NDVI Color Map (right) of Ridgeline with General

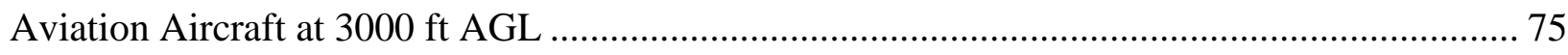

Figure 76: NDVI Payload Integration with IOS CubeSat Kit ................................................... 78 


\section{List of Tables}

Table 1: Overview of Commercially Available Sensors, Distributors, Sensor Principle,

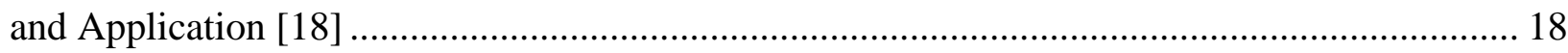

Table 2: Cubesat Startup Companies and Component/Service Providers [34] ............................ 33 


\section{Nomenclature}

\begin{tabular}{|c|l|c|}
\hline Symbol & \multicolumn{1}{|c|}{ Description } & Units \\
\hline \multicolumn{2}{|c|}{ English Symbols } \\
\hline RGB & Red Green Blue & {$\left[\mathrm{in}^{2}\right]$} \\
\hline VIS & Visible & {$[-]$} \\
\hline NIR & Near-infrared & {$[-]$} \\
\hline NDVI & Normalized Difference Vegetation Index & {$[-]$} \\
\hline SR & Simple Ration & {$[-]$} \\
\hline Storage & Memory Storage Capacity & {$[\mathrm{MB}]$} \\
\hline$\Delta \mathrm{T}$ & Duration of Time per Flyby & {$[\mathrm{min} / \mathrm{access}]$} \\
\hline $\mathrm{R}_{\mathrm{b}}$ & Downlink Data rate & {$[\mathrm{Mb} / \mathrm{s}]$} \\
\hline $\mathrm{N}$ & Number of Images Downloaded per Flyby & {$[\mathrm{image} / \mathrm{access}]$} \\
\hline$S_{\text {img }}$ & Size of Image & {$[\mathrm{Mb} / \mathrm{image}]$} \\
\hline $\mathrm{R}_{1}$ & Value of Resistor 1 & {$[\mathrm{k} \Omega]$} \\
\hline $\mathrm{R}_{2}$ & Value of Resistor 2 & {$[\mathrm{k} \Omega]$} \\
\hline $\mathrm{V}_{\text {out }}$ & Output Voltage & {$[\mathrm{V}]$} \\
\hline
\end{tabular}




\section{Acknowledgements}

Firstly, I would like to thank my research advisor Dr. Powsiri Klinkhachorn for his support, wisdom, and above all, providing an unwavering mentorship throughout the entirety of my graduate experience in electrical engineering. His enthusiasm for innovation and passion for research has inspired me to continually work toward enhancing my career as a professional engineer. His guidance has made the completion of this thesis possible. My committee members Dr. Mario Perhinschi and Dr. Roy Nutter also deserve lots of gratitude for their advice and kind efforts in making this thesis a better document.

Many thanks goes to each of my colleagues at the National Aeronautical and Space Administration (NASA) Independent Verification and Validation (IV\&V) Facility for always offering their advice and helping hand as I worked toward completing my research. Specifically, I would like to thank Mr. Greg Lusk for his help in the experimental setup and testing of the custom printed circuit board (PCB) in addition to all his expert advice in electrical engineering and computer technology, Mr. Joel Lindsay for his patience and assistance in becoming familiar with Computer Aided Design (CAD) software, and Miss Abigail Ida for her assistance in graphical design and NDVI analysis of test data.

Finally, I could not have made it this far without the overwhelming support from my family and friends. For being there every step of the way, I cannot thank them enough. 


\section{Chapter 1 | Introduction}

\subsection{Normalized Difference Vegetation Index}

Normalized Difference Vegetation Index (NDVI) is one of the most common techniques used by researchers to measure plant "greenness" or photosynthetic activity. The concept of NDVI is based on the observation that the amount of reflected light from healthy green vegetation varies from stressed or dead vegetation and rocky surfaces. Vegetation that is considered healthy, or photosynthetically active, mostly absorbs red light that falls within the visible spectrum while reflecting a large portion of near-infrared light. On the other hand, stressed or dead vegetation tends to reflect more visible red light and less near-infrared light. For non-vegetated surfaces, the reflectance is much more even across the light spectrum [1]. The variation in amount of absorbed and reflected light of vegetation occurs because the chlorophyll pigment in plant leaves uses the absorbed visible light from 0.4 to $0.7 \mu \mathrm{m}$ in the photosynthesis process. Near-infrared light from 0.7 to $1.1 \mu \mathrm{m}$ is coincidentally reflected by the cell structure of the leaves [2]. Therefore, a heathier plant absorbs more visible light for increased photosynthetic activity and also has a denser cell structure to reflect higher levels of near-infrared light. An illustration of this concept is shown in Figure 1.

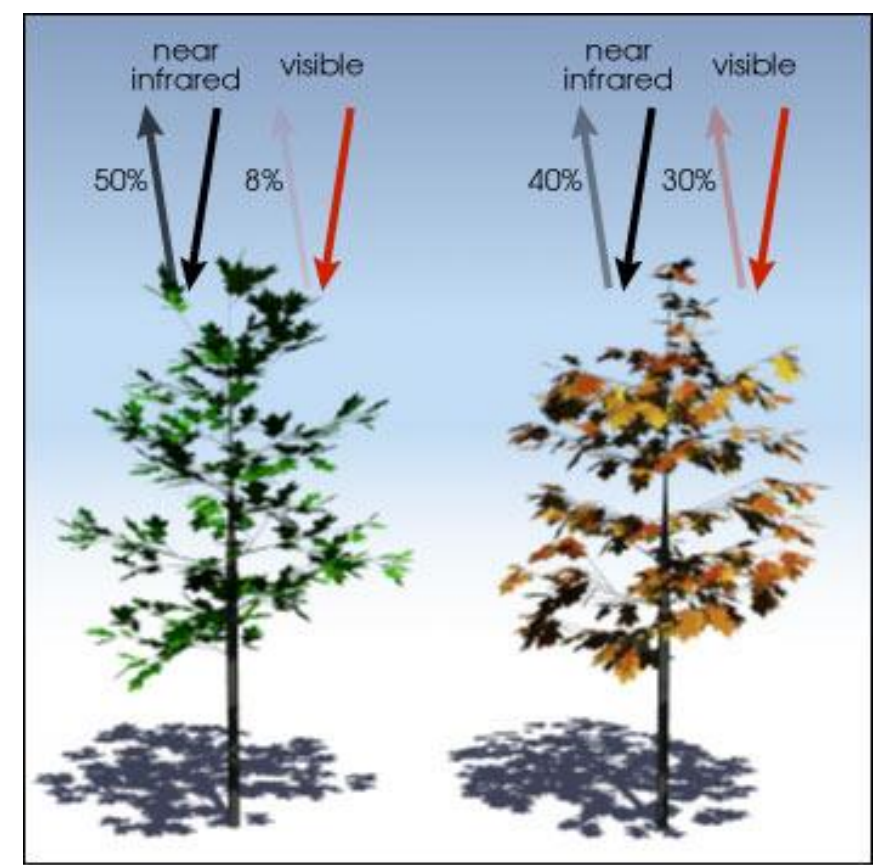

Figure 1: Comparison of Reflected Visible and Near-Infrared Light for Green and Senescent Vegetation [2]

The mathematical formula for calculating NDVI is given by Equation (1): 


$$
N D V I=\frac{N I R-V I S}{N I R+V I S}
$$

where NIR is the intensity of reflected near-infrared light and VIS is the intensity of reflected visible light, also known as red/green/blue (RGB) light. "Nearly all satellite Vegetation Indices employs this difference formula to quantify the density of plant growth on the Earth" [2]. This calculation is typically done on each pixel of images taken by cameras with special lenses to capture the particular wavelengths of interest.

The resulting calculation of NDVI for a given pixel ranges from negative one $(-1)$ to positive one (+1). However, real-world applications usually produce resulting NDVI values that are above zero. Areas of barren rock, sand, or snow typically result in NDVI values that roughly 0.1 or less. Shrubs and grassland or senescing crops could produce results within the approximately 0.2 to 0.5 . Dense vegetation found in temperate and tropical forests or healthy crops at peak growth stage could show NDVI values ranging from around 0.6 to 0.9 [3].

Scientists around the world use this mathematical formula to transform raw satellite imagery data containing information about the reflected light spectra into what is referred to as vegetation indices. Simply put, a vegetation index is a greenness indicator, or an indicator of the relative density and health for each pixel in a satellite image [3]. "Although there are several vegetation indices, one of the most widely used is the Normalized Difference Vegetation Index (NDVI)" [3]. Using NDVI values, researchers can produce images and other artifacts that provide an approximate measure of vegetation type, amount, and land surface conditions. "NDVI is especially useful for continental- to global-scale vegetation monitoring because it can compensate for changing illumination conditions, surface slope, and viewing angle" [3]. There are many specific applications that rely on NDVI as a key to generating the desired results. For example, nominal growing conditions for a particular region and time of year can be established by averaging NDVI values over time. NDVI analysis can also reveal areas where vegetation growth has changed due to human interactions such as deforestation and overgrazing as well as natural disturbances such as wild fires or changes in the phenological stages of plants [3][4][5].

\subsubsection{Applications of NDVI}

Deforestation has proven to inherently produce negative effects on ecological systems for both regional and continental scales despite the gains of lumber and other industries. "Between 2000 and 2012, global forest loss increased by approximately $2000 \mathrm{~km}^{2}$ per year" [6]. Several techniques exist that utilize different Vegetation Indices (VI) for deforestation mapping; however, NDVI is the most frequently used VI for deforestation mapping in the tropics. By comparing current observations against previously captured data, disturbances in forest density can be mapped in near-real time. Deforestation can be accurately detected using the Breaks For Additive Seasonal Trend (BFAST) method which separates the time series into a historic period, which is used as a baseline, and a monitoring period [6]. 
In addition to deforestation, drought and subsequent wildfires also pose a serious threat to many areas of the world which warrants continued research in these areas. A relationship between NDVI, precipitation, and soil moisture has been reported and for this reason it has been extensively used to assess drought and monitor vegetation health. Shallow-rooted and deeperrooted vegetation for many different types of landscapes can be temporally decomposed for monitoring changes in health, particularly important for droughts in semiarid regions and savannahs with mixed trees and ecosystems [6][11]. Droughts are put into four broad classifications: meteorological (lack of precipitation), agricultural (lack of soil moisture), hydrological (lack of run-off water), and socioeconomic (lack of water supply); yet all types of droughts can be attributed to a sustained precipitation deficit [8].

With over two decades of NDVI data covering the entire globe, researchers at the National Aeronautics and Space Administration (NASA) and the National Oceanic and Atmospheric Administration (NOAA) use this 20-year average to establish a typical productivity level for a given region and to then compare to a particular month or even an entire year of interest. "In most climates, vegetation growth is limited by water so the relative density of vegetation is a good indicator of agricultural drought" [6]. Thus, a given region is labeled as "indrought" if there is a noticeable reduction in the amount of vegetation growth due to lack of precipitation. "The difference between the average NDVI for a particular month of a given year and the average NDVI for the same month over the last 20 years is called NDVI anomaly" [6]. In 1993, heavy rain in the Northern Great Plains led to flooding of the Missouri River which resulted in exceptionally lush plant growth in the region, i.e. a positive anomaly. At the same time, precipitation was very low in the Eastern U.S. resulting in levels of vegetation growth that were less than normal, i.e. a negative anomaly. Both of these positive (green) and negative (red) anomalies can be seen in Figure 2.

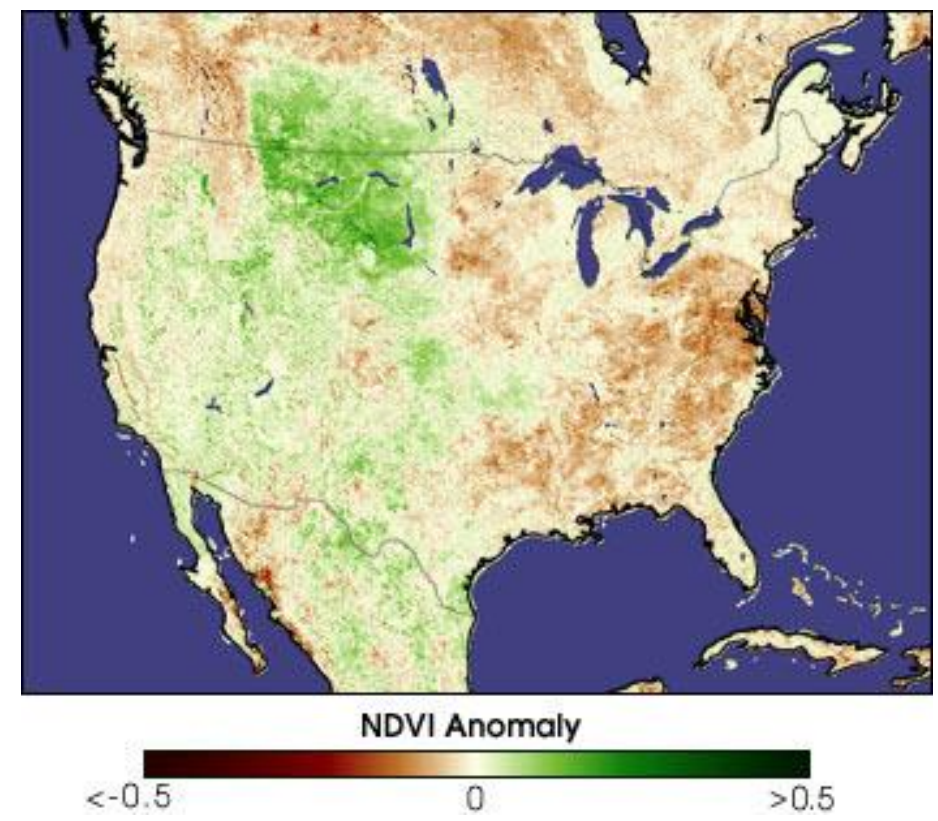

Figure 2: NDVI Anomaly in the U.S. for August 1993 [6] 
Wildfire risk assessment, also referred to as fire potential, is based on the probability that ignition will occur and the potential propagation of the fire, or fire hazard. The fuel moisture content (FMC) in both live and dead biomass is directly related to the probability of ignition [9]. Currently, scientists at the NASA Goddard Space Flight Center are systematically investigating the relationship between wildfire and the water-cycle in sub-Saharan Africa. When satellite data was used to match fire activity to hydrological indicators, a pattern was observed which suggested a tendency for precipitation to be suppressed by the net influence of fire [10]. For example, measurements of key parameters which help trigger rain (soil moisture, evaporation, and green vegetation) decreased in the wet season following years with more than average burning during the dry season. "Even within dry seasons, the amount of water decreased in areas with more humid climates as the burning became more severe" [10]. Though currently these results may only show a correlation between fires and water cycle, the data collected from this study will improve climate models to allow a more direct relationship between the burning of biomass and the impacts of wildfires on drought.

With the global population at a maximum and still on the rise, shifting dietary preferences, and massive food wastage, the demand for food is steadily increasing. According to the Food and Agriculture Organization (FAO), an estimated 70\% increase in global food production will be necessary to meet demands in 2050, though some experts argue this is still an underestimate [12]. Urgency for global food provisioning will be without a doubt a concern world-wide in the decades to come and demands a variety of creative solutions and policies at various levels from local, regional, to global. "One of the most urgent responses to this situation must be measures to stop and reverse land degradation" [12]. Back in 1994, the United Nations Convention to Combat Desertification (UNCCD) recommended eleven impact indicators for monitoring long-term land degradation. NDVI is proposed as a measure of land cover status, one of these key impact indicators. Figure 3 illustrates the principle of hysteresis on plant productivity due to a disturbance, such as drought, grazing, or land degradation. Before the stress at point $A$, the productivity is high. When the stress is at a maximum, the productivity has declined to point $B$. The green curve represents a fully resilient system that springs back to its original productivity rate at point $A$. However, the red curve represents a less resilient system that recovers to a lower productivity rate at point $C$. The resilience of the system, $R$, is described by the distance between $A$ and $C$. "NDVI can be useful in the evaluation of vegetation cover, carbon stocks, and land condition, which may provide resilience indicators" [12]. 


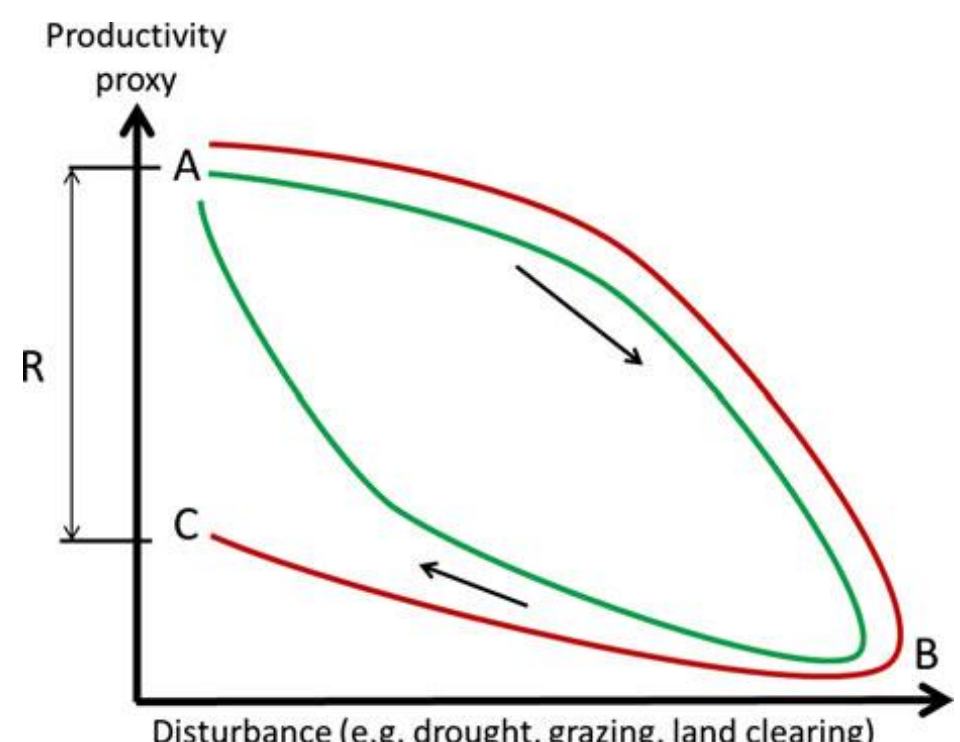

Figure 3: The Principle of Hysteresis [12]

Although it has been shown that NDVI is quite useful in studying vegetation from an ecological perspective, it is also beneficial for many agricultural, residential, and biological applications. For example, NDVI can be used to determine vegetation water content (VWC) to infer water stress for irrigation decisions and aid in yield estimations for crops such as corn and soybeans [13]. Furthermore, there is an increasing interest in neighborhood "greenness", or exposure to vegetation and green space, and its association with positive effects on physical and mental health and reduced risk of cardiovascular issues. "NDVI seems to be a valid and practical metric for use in epidemiological research of associations between neighborhood-level greenness and health" [14]. From a biological perspective, NDVI has recently allowed the connection between distributions of animal species and resources available to be better understood. In 2011, it was shown how the distribution of a lek-breeding population of topi antelopes relates to the abundance of resources before and during the rut [15]. These examples only provide a subset of NDVI applications but clearly demonstrate its versatility and usefulness in solving real-world problems.

\subsubsection{Remote Sensing}

Research has shown that multiple NDVI applications exist for ground-based experiments and general hobbyists. The leaf area index of vegetation can be estimated, temporal dynamics of canopy structure and phenology can be studied, and even simple yet practical methods such as detection of unwanted weeds in specific sites can be performed using ground-based NDVI measurements [15][17][18]. However, it can be argued that the most practical use of NDVI is through the use of remote sensing. This is largely due to the wider coverage of surface area that can be analyzed with remote sensing. The most common methods used to gather remote-sensed 
NDVI data is through the use of unmanned aerial vehicles (UAVs), e.g. rotocopters and/or conventional fixed-wing aircraft, and via numerous satellites with sophisticated sensor arrays orbiting the earth.

Though other vehicles can also be used to capture NDVI images remotely, such as general aviation aircraft and high-altitude weather balloons, the use of quadcopters and other micro UAVs for localized or site-specific studies are becoming increasingly popular amongst both researchers and general hobbyists [19][21]. Figure 4 shows the NASA Sensor Integrated Environmental Remote Research Aircraft (SIERRA) at the NASA Crow's Landing airfield in California. The data collected by SIERRA has enabled extensive environmental studies on flood monitoring, wildfires, volcanic activity, fault line mapping, and the exploration of geothermal and other natural/mineral resources.

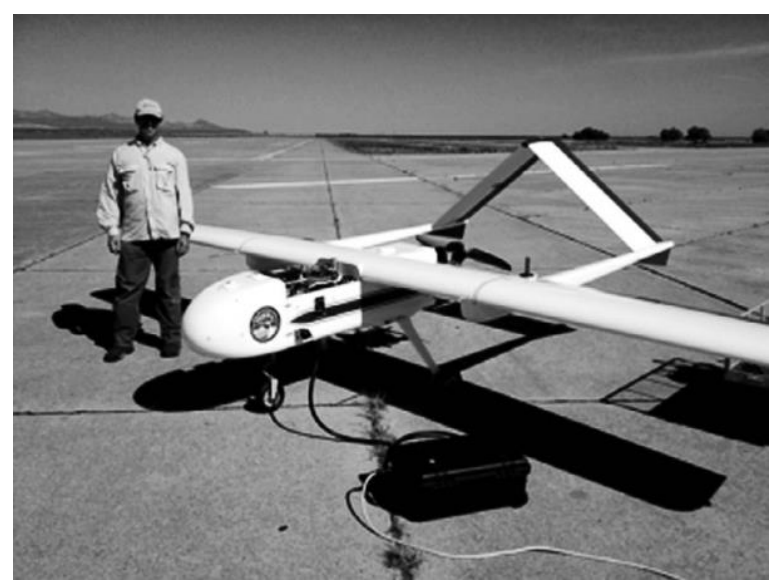

Figure 4: SIERRA (Wingspan $=6 \mathrm{~m}$ ) on the Tarmac at NASA's Crows Landing Airport in California [19]

In Figure 5, a typical unmanned helium blimp is demonstrating the capability for remote imaging with its camera rig in flight. The blimp shown has a fuselage length of approximately $4 \mathrm{~m}$ and is constructed of a durable single-layer urethane material. The four rigid tail fins provide in flight stability for the blimp and the keel provides multiple attachment points to fasten the tether lines suspending the camera package [19]. 


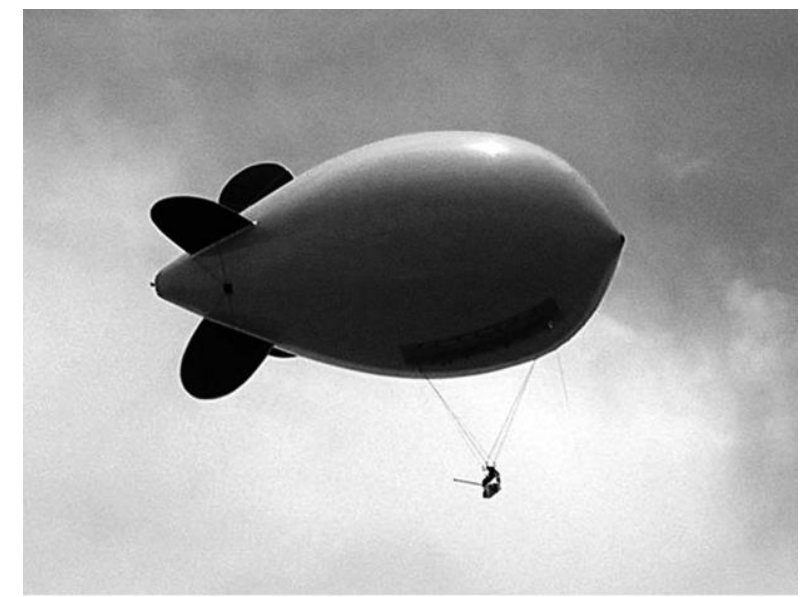

Figure 5: Helium Blimp $($ Fuselage $=4 \mathrm{~m})$ with Camera Rig in Flight [19]

"UAVs can fly at low altitudes and take ultra-high spatial resolution imagery, which allow observing small individual plants and patches that is not possible with satellites or piloted aircraft" [21]. Quadcopters and other rotocopters employ a vertical takeoff and landing (VTOL) system which allows them to be independent of a runway. As a result of VTOL capability, these platforms can be used in a wide range of various situations and landscapes. In Figure 6, an image of the MD4-1000 quadcopter during a flight test over a sunflower field at La Monclova Farm in La Luisiana, Seville, Spain is shown. This study focused on early site-specific weed management (ESSWM) applications. The effects of flight altitude on image special and spectral solution were also analyzed [21].

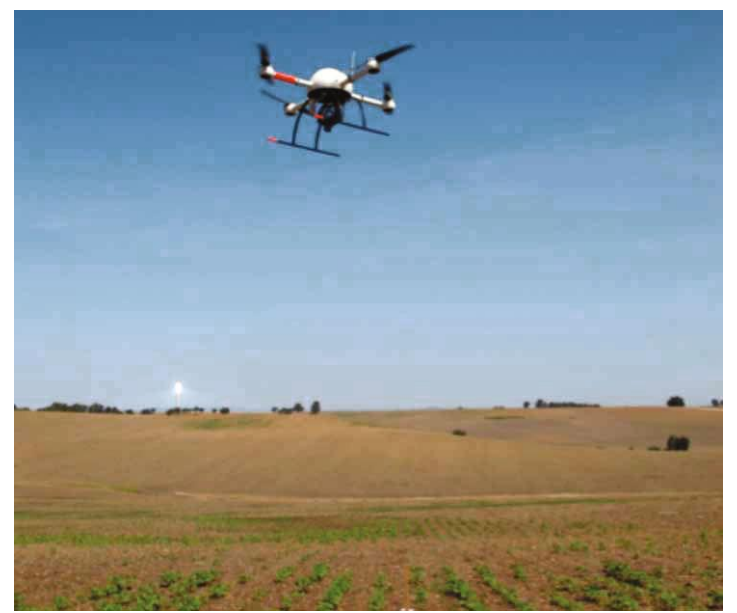

Figure 6: Flight Testing of Quadcopter UAV (Model MD4-1000) over Experimental Sunflower Crop-Field [21]

As illustrated, UAVs are effective in gathering NDVI data for localized or site-specific situations. However, for wide area coverage on regional and continental scales the most practical method of remote sensing is to launch satellites or a series of satellites into strategic orbits with 
high-resolution imaging and filtering apparatus. For example, the Moderate Resolution Imagine Spectroradiometer (MODIS) sensor suite aboard the NASA Terra and Aqua satellites produce vegetation indices on 16-day intervals at various spatial resolutions which allow consistent comparisons of vegetation canopy greenness. Leaf area, chlorophyll concentrations, and canopy structure attributes can then be studied from this composite property.

The vegetation indices use a specific compositing algorithm to remove low quality pixels, then from the remaining good pixels the two highest NDVI values are determined and the pixel closest to nadir is selected to represent the compositing period. This MODIS-specific VI algorithm yields a phased product with each 16-day composite generated eight days apart. Because the MODIS sensors aboard the Terra and Aqua satellites are identical, combining both data records allows for a higher temporal resolution product [23]. "The MODIS VI product suite is now used successfully in all ecosystem, climate, and natural resources management studies and operational research as demonstrated by the ever increasing body of peer publications" [23]. In Figure 7, a sample of the MODIS/Terra NDVI 16-day composite has been pseudo-colored to represent the health of vegetation biomass across western United States.

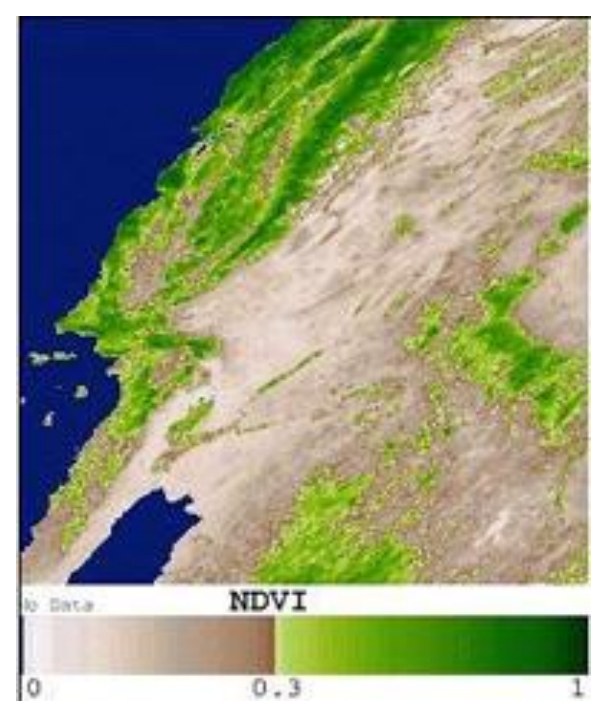

Figure 7: Sample of the MODIS/Terra 16-day NDVI Composite Product (June 25- July 10, 2000) [23]

Another example of NDVI data collected via satellite imagery is the NASA Landsat missions. Since 1972, this fleet of NASA Earth-observing satellites has provided the world with the longest unbroken data stream of land coverage changes viewed from space and subsequent effects on Earth [24]. The contribution of over 40 years of continuous Landsat data has permitted crucial "research on climate, carbon cycle, ecosystems in water cycle, biogeochemistry and changes to Earth's surface, as well as our understanding of visible human effects on land surfaces. Building off that research, the Landsat imaging data set has, over time, led to the improvement of human and biodiversity health, energy and water management, urban planning, 
disaster recovery and agriculture monitoring, all resulting in incalculable benefits to the United States and world economy" [24].

In 2012, a drought affected large areas of the western United States. A specific case illustrating the usefulness of NDVI with remote sensing can be seen by the images of the drought on the Cheyenne Bottoms Wildlife Wetlands area in central Kansas captured by Landsat-7 in Figure 8. "As the largest interior marsh in the United States, Cheyenne Bottoms provides a resting place for millions of migrating birds every fall. As the water levels dwindle and disappear, wildlife officials are concerned that the effects could be devastating for the habitat" [25]. In the leftmost image, a sufficient amount of water is visible in the wetland area. Over the span of just two years from 2010 to 2012, the water levels visibly continued to drop until virtually all the water had evaporated from the natural habitat. As cases like these continue to threaten our wildlife today, the collection of Landsat data is extremely useful to officials for making decisions on what action to take in effort to sustain habitats for wildlife [25].

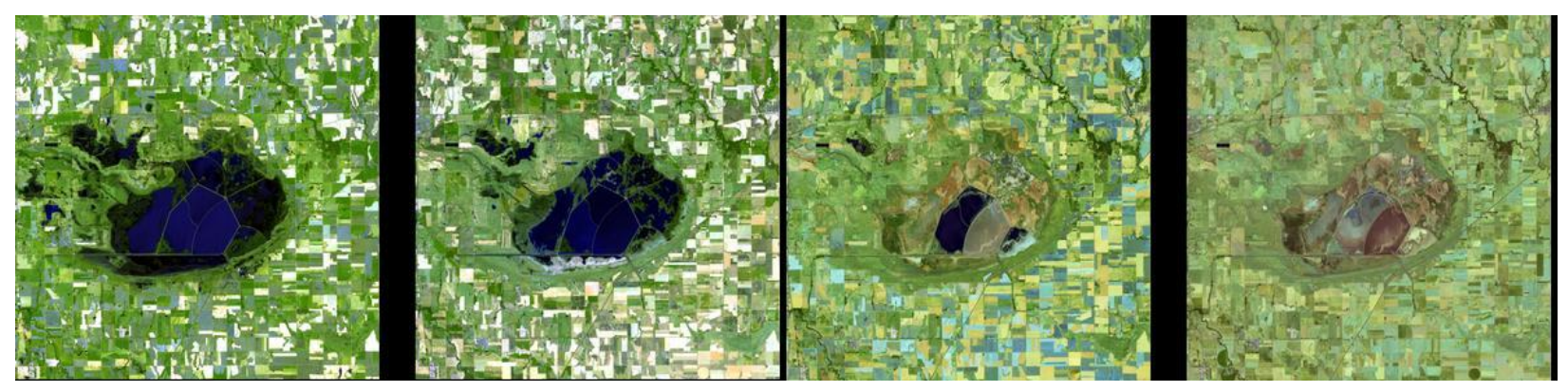

Figure 8: Effects of Drought Captured by the LandSat-7 Satellite [25]

\subsection{CubeSat Space Systems}

The science return from large Earth-observing satellites was proclaimed to be tremendous based on the concept that a variety of measurements could be gathered and correlated from a single platform. A reduction of payload cost per $\mathrm{kg}$ was also expected as several experiments shared a single communication bus and launch vehicle [26]. However, these cost reductions do not seem to materialize fully in reality due to a multitude of engineering and programmatic problems that typically emerge during development which increased cost. It is likely that NASA and ESA have created programs based on smaller satellites as a result of this problem. From an engineering perspective, the technical issues related to having several instruments integrated on a common platform can be avoided with smaller development projects based on single-instrument satellites. "Furthermore, programs based on smaller satellites have desirable programmatic properties, for example in terms of robustness to schedule slippage, to launch failure, or to budgetary instability" [26].

The CubeSat platform, co-developed by Professor Jordi Puig-Suari at Cal Poly and Professor Bob Twiggs at Stanford, has been proposed as a viable spacecraft platform for future 
scientific missions and various surveys of Cubesats and other miniature satellites are available in the literature. Miniature satellites have been used in recent years for technology demonstration as well as for education. "In the education world, several universities around the world have started nanosatellite programs to teach their students the fundamentals of satellite engineering and project management as well as to provide them with excellent hands-on experience" [26].

Although most academic Cubesat programs hold science as a secondary objective while maintaining education or technology demonstration as the primary objective, the potential quality of science that can be achieved with Cubesats has surprised researchers in some cases. "The advent of the CubeSat standard has allowed academia, with minimal investment to go through the process of designing, building, and integrating spacecraft in a relatively short time period. These programs provide an unparalleled opportunity to learn the practical aspects of spacecraft engineering" [27]. It is without doubt that students who are exposed to these types of development opportunities are better prepared for professional industry. Figure 9 illustrates a simplified organizational chart commonly adopted for a space system development program. All of these areas are necessary support functions of a typical CubeSat development project and provide a great opportunity for young engineers and technicians to gain field experience and interaction with other key members of the team throughout a full lifecycle development process.

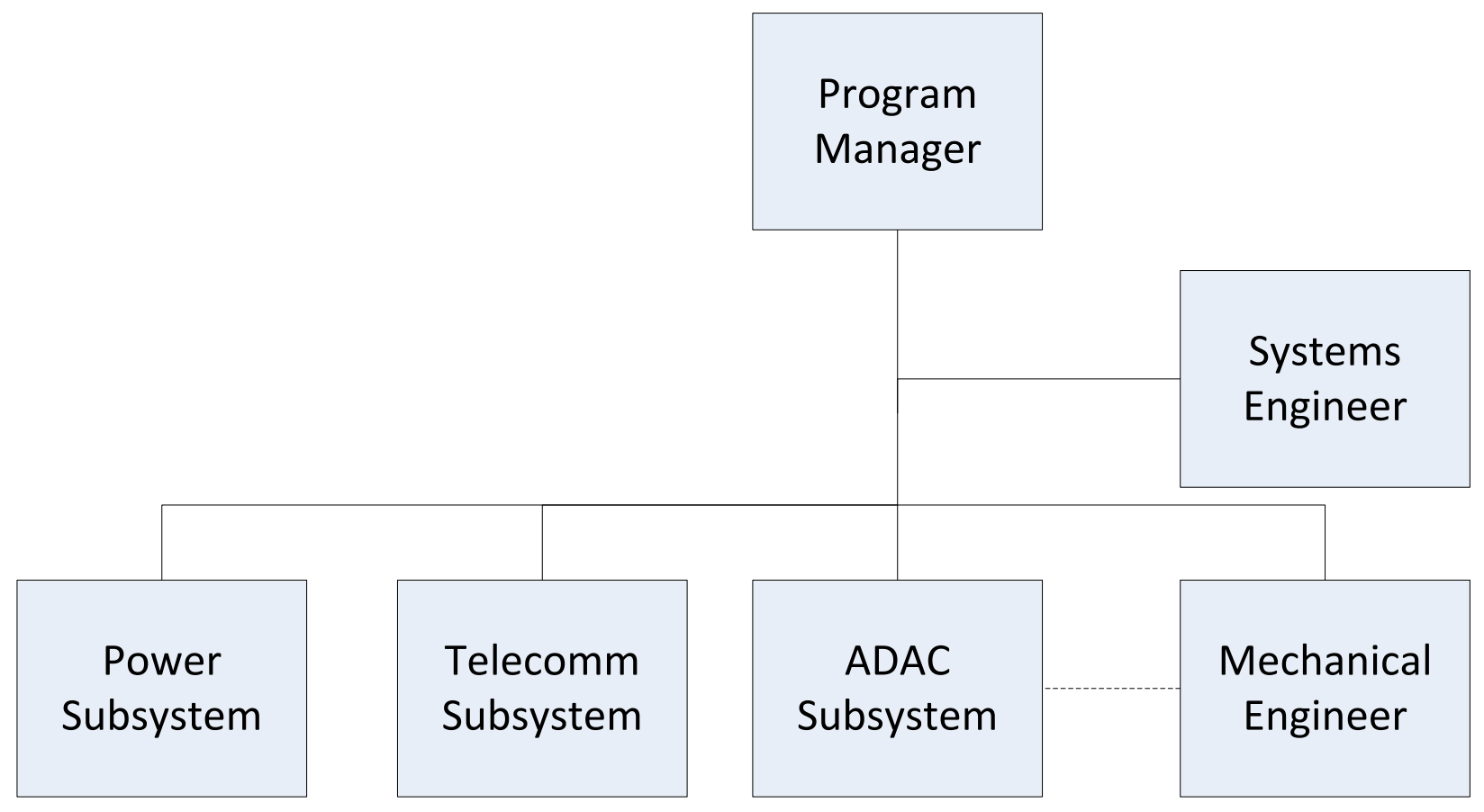

Figure 9: Simplified Subset of a Typical Organization Chart for a

Complex Space System Development Program [27] 


\subsection{Research Objective}

Two major obstacles for academia and other newly established space programs in building payloads for atmospheric and space applications are software development and hardware assembly. Successful mission operations depend on properly written code to maintain critical systems and protect from data corruption. Software is just as important for the successful operation and management of Cubesat payloads as it is for larger satellite systems. "Cubesats provide low-cost platforms for gaining practical, real-world experience in software algorithm development, understanding of real-time data management, control-theory, and softwarehardware interfaces. This expertise is directly transferable to many job skill sets in the information technology (IT) market" [34]. However, the cost of developing software on proprietary systems can become very expensive, especially when additional development support is needed for implementing custom software functions. Consequently, the use of open-source software or freeware is becoming more popular in smaller, lower class development projects. Moreover, the assembly of surface mount electronics and processor chips directly onto a printed circuit board (PCB) can be quite challenging for electrical engineers and technicians that do not have experience working with embedded systems. The proposed experimental payload will provide a low-cost, open-source software solution with a simple commercial off-the-shelf (COTS) hardware design for NDVI analysis from low altitude to upper atmosphere and space applications.

The underlying objective of this thesis is to offer proof-of-concept of a low-cost scientific payload design that is compatible with cubesat development kits, such as the kits offered by IOS and Pumpkin, Inc., for public use in the advancement of space-based systems development for the next generation of scientists and engineers [36][44]. The proposed payload design concept incorporates COTS components to keep costs affordable and to prove the capabilities and characterize limitations of using COTS devices. A power circuit was designed as part of the payload board to boost and regulate voltage supplied by a $3.7 \mathrm{~V}$ lithium-ion battery to $5 \mathrm{~V}$. The NDVI experiment was selected due to its feasibility in Earth science studies over a wide range of applications and use cases. Open source software was also developed to reliably capture images from two cameras simultaneously at a specified rate. While the use of COTS components may not provide the resolution or transmission rates that may be necessary to gather game-changing science at orbital altitudes, it could still provide a level of scientific return that supports academic growth and learning on an achievable budget for most educational programs. Simply gathering and the results obtained from an orbital mission and analyzing the quality of the results provides a tremendous opportunity for knowledge gain and experience, and undoubtedly cultivates positive learning regardless of data resolution.

This thesis provides an experimental design and method for performing NDVI analysis at various altitudes by taking simultaneous images with a normal camera lens for visible light (VIS) and with a near-infrared (NIR) camera lens. It will be shown that the experimental setup is capable of remotely capturing usable imagery for NDVI analysis. In order to operate in the harsh conditions of the upper atmosphere and space environment, the system will be required to 
withstand extreme temperature fluctuations and be resilient to a wide range of vibrations. The precision of the results will be limited to the resolution of the COTS camera lenses selected for the payload design. Quadcopter and general aviation aircraft flights at various altitudes are used to verify proof of concept. Image processing software was used to generate NDVI maps of landscape and to qualitatively assess vegetation health. Considerations for flights on highaltitude balloons and suborbital launch opportunities are also discussed for implementation into future work.

\subsection{Thesis Organization}

An in depth literature review of various terrestrial and space applications of NDVI and an overview of past and current Cubesat missions are presented in Chapter 2. The experimental design, software design, and PCB design are explained in Chapter 3, including mechanical and electrical layouts. A detailed set of instructions for soldering, payload assembly, and wiring connections to build the proposed experiment with basic COTS components is also given in Chapter 3. The results of post-processing analysis and experimental flight testing are shown in Chapter 4. Finally, conclusions and recommendations are provided, future work is discussed, and lessons learned are summarized in Chapter 5. 


\section{Chapter 2 | Literature Review}

This section summarizes relevant background information and history related to remote sensing NDVI and Cubesats. Various scientific examples that apply NDVI testing on the earth's surface and space missions are discussed. In addition, an explanation of the successes and failures of Cubesats and recent academic advancements in space development are given. The end of the chapter will conclude with the objective of this thesis

\subsection{Terrestrial Applications of NDVI}

\subsubsection{Ground-based NDVI}

There are numerous applications of ground-based NDVI measurements that can be employed to analyze vegetation growth for agricultural and plant science studies. For example, a study by Ponteiller, et al [15] made use of a hand-held NDVI device to estimate leaf area index (LAI). LAI is an important parameter in modeling vegetation productivity and plays a key role in understanding the ecosystem. Regardless of the method used to determine LAI, the process is time consuming and somewhat inaccurate. Furthermore, LAI must be determined frequently using nondestructive means, especially for long term experimental sites. [15]. "NDVI is probably the most commonly used index for analyzing vegetation on a continental scale. This spectral index makes use of radiance or reflectances in NIR and R spectral regions because they enhance the contrast between soil and vegetation. Such an approach should provide satisfactory estimates of LAI rather than plant area index (PAI) because only green parts are taken into account" [15].

A cross-section of the hand-held NDVI device is shown in Figure 10. The hand-held device was designed to measure the damping ratio of the radiance. To produce the highest resolution, measurements should be collected on sunny days keeping a constant sun elevation of at least $40-45^{\circ}$ between data samples. This means that in February data should be collected at solar noon and gradually moving up to 3.5 hours before or after noon in June [15]. 


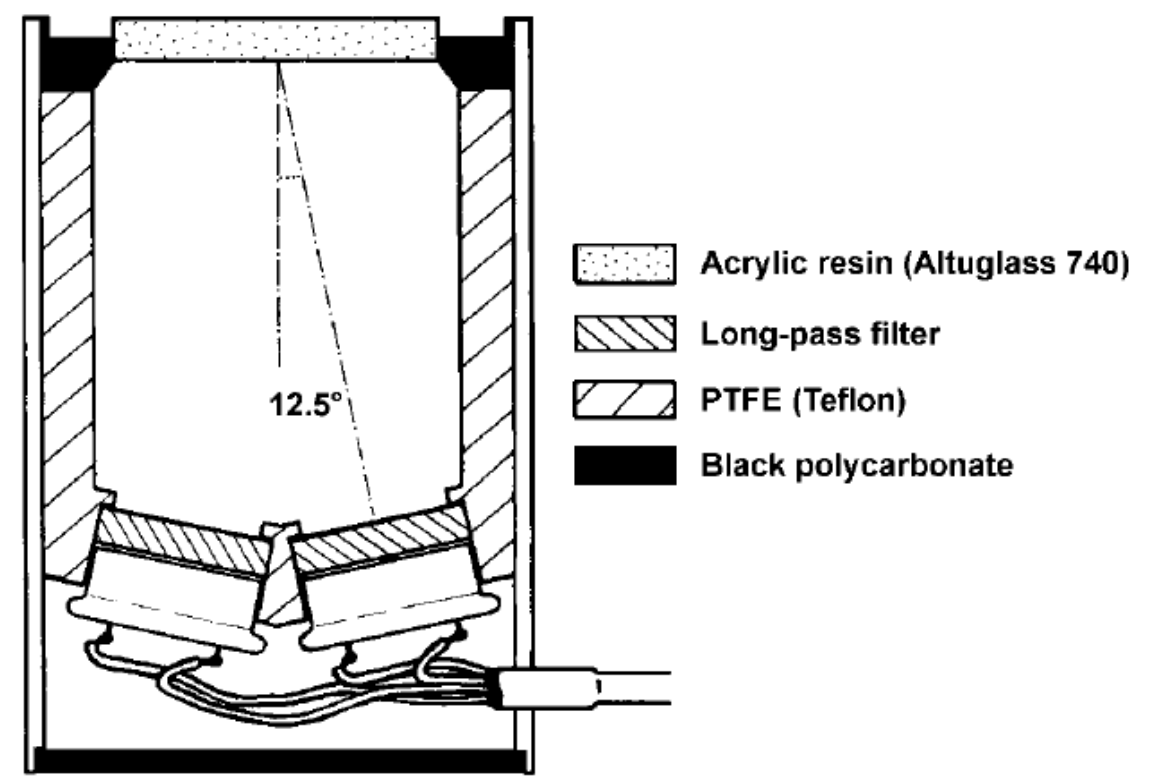

Figure 10: Longitudinal Cross-section of NDVI Hand-held Device (55 mm length, 43 mm dia.) [15]

Unlike other indirect field techniques, NDVI measurements sense predominantly green plant parts and disregards woody areas. Where it may be hard to implement other techniques, e.g. in the case of low canopies, NDVI is easily adapted. "In situ NDVI measurement is not a new technique, but it has not been widely used to date. This is probably due to the lack of a simple portable instrument" [15]. Moreover, errors caused by atmospheric conditions are less of an issue for in situ NDVI measurement than for measurements taken from space [15]. By collecting in situ NDVI and comparing against corresponding space-borne measurements, accuracy of the data can be verified thereby increasing confidence in the overall NDVI results. The proposed NDVI design is well suited for in situ applications and could also be used for space-borne measurements.

NDVI measures the red radiance in the visible light spectrum and NIR radiance above the canopy. Due to its ability to sense small changes in photosynthetic activity of vegetation, NDVI is commonly used in remote sensing studies [17]. A study done in 2012 employed a network of forest and herbaceous sites that are equipped with laboratory made NDVI sensors to monitor the canopy structure daily for temporal dynamics and phenology over the past $10+$ years. The network of NDVI measurements is not only important for phenological studies but it also provides calibration data needed to validate direct satellite observations. "NDVI sensors were installed on towers, a few meters above the top of the canopy and looking downwards. They were generally inclined at about $20-30^{\circ}$ from vertical and oriented towards south to avoid hotspot effects in canopy reflectance when the viewing direction is collinear with the solar direction" [17]. The effects of shadow on NDVI are minimized around solar noon where the level of direct solar radiation on the canopy top is highest and changes in the sun angle are smallest. It can be seen in Figure 11 that the global radiation ratio is above 0.6 for totally clear skies. Thus, this value can be used to eliminate cloudy periods as long as it is assumed to be the 
same for all sites regardless of climate. "The effects of cloud cover are strongly reduced since only NDVI data acquired under clear skies or low cloud fractions are considered to construct NDVI time-series" [17].

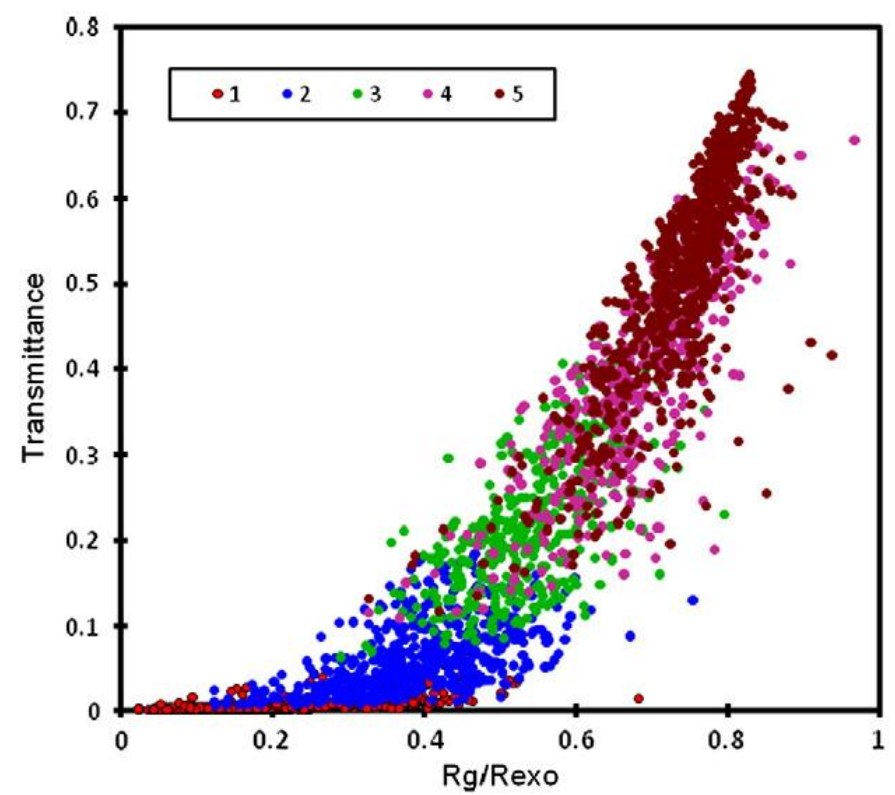

Figure 11: Solar Transmittance vs Ratio of Global Radiation at Ground Level (Rg) to Top of Atmosphere (Rexo) in 2019 for Five Levels of Sunshine (1-overcast, 5-clear sky) [17]

A direct example of how ground-based NDVI can be analyzed for phenological studies of agriculture is illustrated in Figure 12. At the African savanna site, NDVI is at its peak at the start of 2008 which aligns with the wet season. As rainfall decreases, NDVI falls in parallel to reach its first minimum during a brief dry period around the 60 day mark. NDVI increases again with the return of rain and reaches another local maximum around the 150 day mark which coincides with the end of the wet season. With the start of the main dry season, NDVI gradually declines again until the field was caught on fire around the 180 day mark causing a sudden drop. After the fire is burnt out, a negative NDVI value is induced from ash and burnt clumps of grass covering the soil. A negative NDVI can be observed throughout the entire dry season up until about day 270 which marks the start of the wet season when vegetation growth becomes active once more. The temporal pattern of NDVI in 2009 is quite similar to that observed in 2008, except that NDVI remains high with a steady decline through the 60 to 120 day mark. This is mostly explained by the amount of rainfall received in that season [17]. 


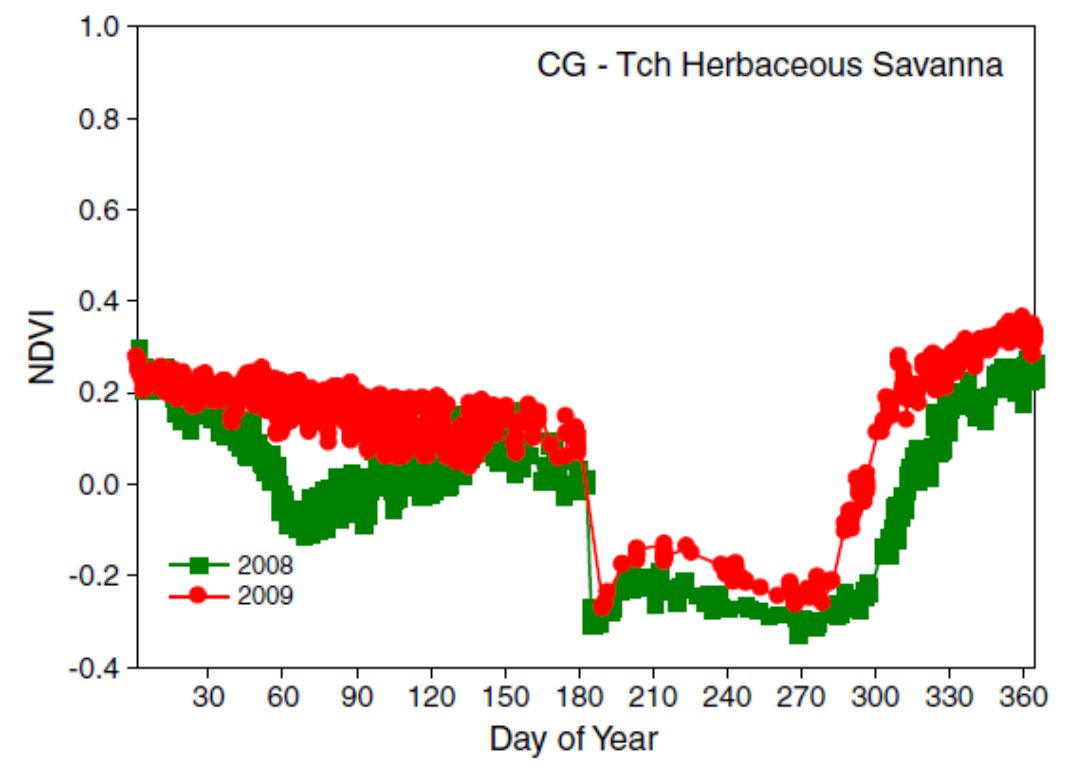

Figure 12: Ground-based NDVI Time-series over Congo African Savanna [17]

Another example of ground-based NDVI analysis for agricultural studies is shown in Figure 13. NDVI measurements at the Belgian agricultural site started during the growth of winter wheat around day 60 in 2007. An increase of NDVI can be seen during the spring reaching a maximum around the 120 day mark, then a steady decline up to about the 180 day mark where a much steeper slope is observed up to a harvest season around day 210. Due to large re-growth of wheat and weeds in early September, NDVI increases once again to reach a small peak centered on day 247. NDVI drops near zero again after the wheat stubble has been plowed off from day 260 on and then rises slightly during the late fall up until the start of winter. In 2008, an increase in NDVI can be seen just a few days after the sowing of sugar beet around day 140 and reaches a maximum around day 210 at the end of July. NDVI remains almost constant throughout the summer until day 308 when the sugar beet is harvested. A few days after harvest, the drastic decline in NDVI is evident. "In, 2009, NDVI time-course within the year is bimodal, reproducing the temporal variation of above ground biomass of wheat and mustard, respectively" [17]. 


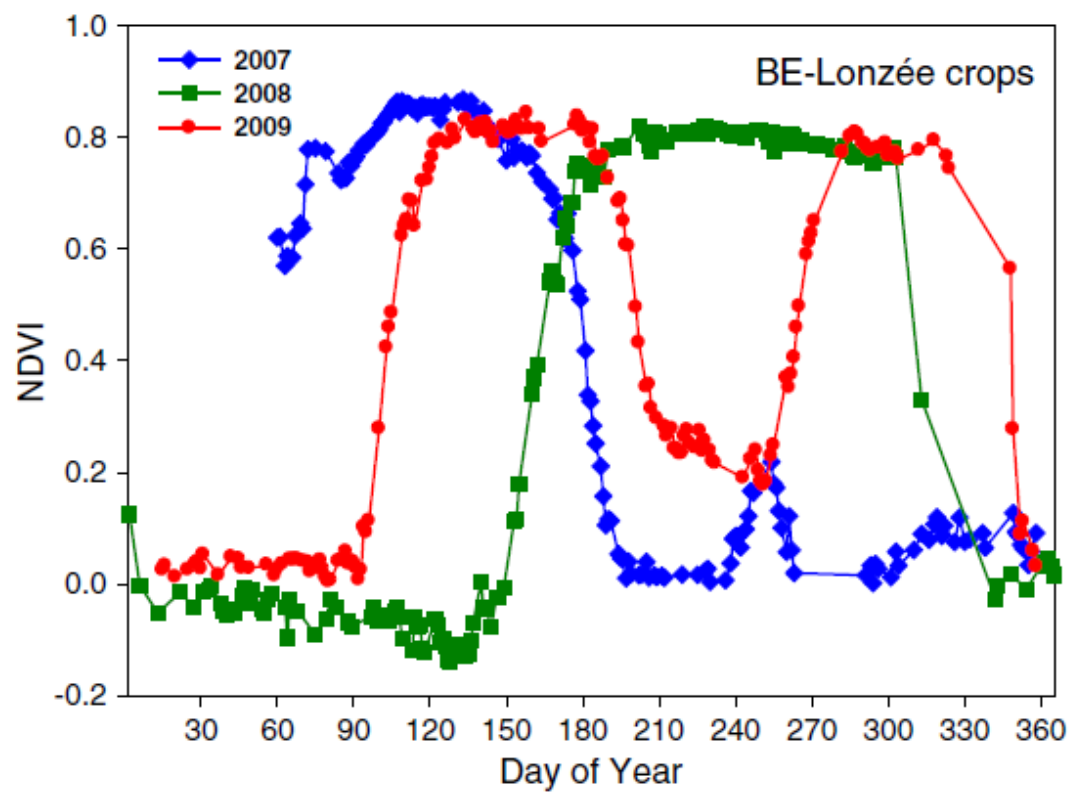

Figure 13: Ground-based NDVI Time-series over a Succession of Crops in Belgium (2007-Winter Wheat, 2008-Sugar Beet, 2009-Winter Wheat and Mustard) [17]

Both of these examples, among others, suggest that the use of a single sensor looking downward is an effective solution for collecting high quality NDVI temporal data that describes canopy greenness with sufficient resolution. From an operational standpoint, this solution is robust, potentially maintenance-free, and well adapted to routine measurements. "However, the absence of a full atmospheric correction affects the accuracy of multi-temporal or multi-site comparisons of NDVI time-series or when converting NDVI data into biophysical variables such as LAI, fraction of absorbed radiation, or the fraction of vegetation cover" [17].

Yet another type of ground-based application of NDVI for use in agricultural studies is for site specific weed management (SSWM). "Site specific weed management is the part of precision agriculture (PA) that tries to effectively control weed infestations with the least economical and environmental burdens" [18]. Weed management on a site-specific scale is achieved by using ground-based or near-range sensors in combination with decision rules and precise application techniques. Over the last three decades, near-range sensor technologies developed for mounting on a vehicle have been emerging for use in field science. "These technologies focus on identifying plants and measuring their physiological status with the aid of their spectral and morphological characteristics" [18]. Machine vision systems have been developed by many researchers to detect weeds in agricultural fields. Some of vision systems can identify weed patches based on the principle that weed infested areas contain more biomass, and can also distinguish crops from different weed species with varying success. The amount of field area that needed herbicides applied was reduced by up to $50 \%$ using site-specific weed control in cereals based on NDVI mapping. Moreover, a 20\% saving of herbicide has been reported by 
varying the application rate from $50 \%$ in areas with very little to no weeds up to $100 \%$ in areas with high weed infestation [18].

These examples of near-range NDVI sensors mounted to ground vehicles provide objective evidence of its practicality for precision agriculture (PA). "In order to support PA operations, vehicle-mounted sensors should be easy to use, with a low-cost for obtaining and maintaining the sensor and a signification duration period for the sensing system" [18]. The proposed NDVI payload configuration has a price tag of around \$100 USD for all components, free maintenance costs, and a reasonable lifespan projected to outlast improvements in technology which warrant an upgraded version to replace it for normal operations. Some existing commercial products are listed in Table 1, along with the distributor name, sensor principle, and application used. A photograph of a ground vehicle sensor setup can be seen in Figure 14 which shows some of the existing commercial sensors integrated on the ground vehicle. The proposed NDVI payload design could be easily implemented in this type of setup.

Table 1: Overview of Commercially Available Sensors, Distributors, Sensor Principle, and Application [18]

\begin{tabular}{|c|c|c|c|}
\hline Sensor & Distributor & Sensor Principle & Applications Used \\
\hline \multirow[t]{2}{*}{ Yara N-sensor } & Yara GmbH \& Co. KG, D“ ulmen, Germany & Spectrometer & Measurement of nitrogen levels through indexes \\
\hline & http://w w w .yara.de & & \\
\hline \multirow[t]{2}{*}{ Crop Circle ACS-470 } & Holland Scientific Inc., Lincoln, NE & Spectrometer & Measurement of green biomass and leaf area estimates \\
\hline & http://hollandscientific.com/ & & \\
\hline \multirow[t]{2}{*}{ Weedseeker } & Trimble Agriculture, Sunnyvale, CA, USA & Optoelectronic sensor & Real-time spot spraying \\
\hline & http://w w w .trimble.com/agriculture/ & & \\
\hline \multirow[t]{3}{*}{ WEEDit } & gps-Ag Pty Ltd, Kangaroo Flat, Australia & Optoelectronic sensor & Real-time spot spraying \\
\hline & http://w w w.w eedit.com. & & \\
\hline & au/w w w.w eedit.com.au & & \\
\hline \multirow[t]{2}{*}{ Multiplex } & Force-A, Orsay, France & Fluorescence sensor & Measurement of photosynthesis activity, \\
\hline & http://w w w .force-a.eu/ & & flavonol content, nitrogen state \\
\hline \multirow[t]{2}{*}{ MiniVegN } & Fritzmeier Umwelttechnik GmbH \&Co. KG, Germany & Fluorescence sensor & Measurement of photosynthesis activity \\
\hline & http://w w w .umw elt.fritzmeier.de/ & & \\
\hline \multirow[t]{2}{*}{ PAM } & Heinz Walz GmbH, Germany & Fluorescence sensor & Measurement of photosynthesis activity \\
\hline & http://w alz.com/ & & \\
\hline Robocrop & http://w w w .garford.com & & hoeing of transplanted crops \\
\hline \multirow[t]{2}{*}{ Robovator } & F. Poulsen Engineering ApS, Denmark & Image sensor & Alignment of cultivators of intra-row \\
\hline & http://w w w .visionw eeding.com & & hoeing of transplanted crops \\
\hline
\end{tabular}




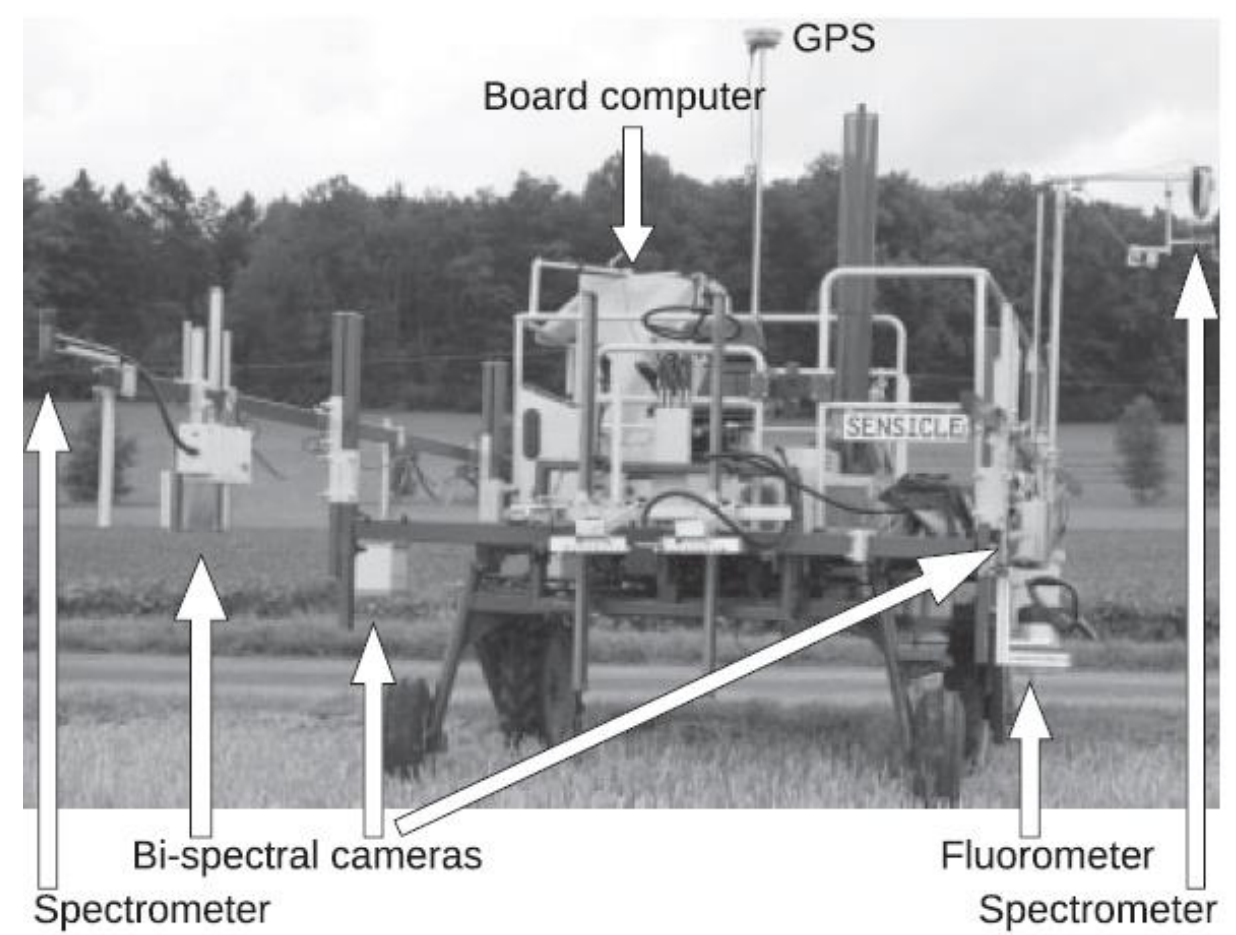

Figure 14: 'Sensicle’ Ground Vehicle Platform with Various Sensors Integrated [18]

Using robots for field scanning and small scale plant nursing operations has been proposed by many researchers. "Robotic systems can be seen as a recommendable platform to implement SSWM, since they include the whole procedure of sensing, decision making, and controlling actuators. They usually provide autonomous navigation procedures, but field and crop characteristics (e.g. row crops) can contribute to navigational purposes" [18]. However, there are several challenges that still drive high costs of robotic implementation, e.g. the volume required for autonomous spraying in an arable crop and safety precautions with unmanned vehicular activity.

\subsubsection{UAVs for NDVI}

The type of platform used for remote sensing is driven by resolution requirements and cost limitations. Remote sensing platforms can range from helicopters and blimps at relatively low altitudes, to aircraft and balloons flying from mid-high altitudes, to satellites several hundreds of $\mathrm{km}$ away. Unmanned aerial vehicles (UAV's) provide a low-cost practical alternative to conventional platforms for remote-sensing of high-resolution data with increased flexibility of operations [19]. UAVs refer to a variety of semi-autonomous or fully-autonomous fixed-wing aircraft, quadcopters and other multi rotor helicopters, and balloons/blimps of varying shapes and sizes with advanced designs to carry small payloads and integrated flight control systems. "Quadcopters and balloons fill a gap between satellites and aircraft when a stationary monitoring platform is needed for relatively long term observation of an area" [19]. 
Satellites offer a large area coverage, multispectral imaging, and have traditionally demonstrated reliable revisit times for studies focused on environmental impact, yet their spatial resolution was still not adequate for many applications. "High spatial resolution satellite data are now available, but the high resolution and frequent, flexible overflights offered by airborne sensors are more suitable for a wide range of applications, such as land use mapping, wetlands mapping, coastline delineation, LIDAR bathymetry, and tracking oil slicks" [19]. For example, the detection of submerged aquatic vegetation (SAV) can be improved by timing an aircraft overflight to view a coastal wetland during low tide. A low-altitude aircraft can accurately map wetland, SAV, and coral reef by integrating imagery with GPS information to be used as layers in a Geographic Information System (GIS) [19]. However, the use of manned aircraft overflights can become quite expensive whereas the cost drops tremendously with the use of UAVs. "GPSguided UAVs have the capacity to obtain very high spatial resolution $(10 \mathrm{~cm})$ imagery of specific landscape features with revisit times determined by the operator as opposed to fixed satellite revisit times. As a result, UAVs such as drones, quadcopters, balloons, and blimps are now being used effectively in many environmental studies" [19].

As previously mentioned, the SIERRA midsized UAV, designed by the U.S. Naval Research Laboratory and developed at the NASA Ames Research Center, is shown in Figure 4. This drone has a wingspan of $6 \mathrm{~m}$, is capable of carrying up to $45 \mathrm{~kg}$ in an exchangeable payload space, and has a range of about $1,000 \mathrm{~km}$ (10 hours). It can fly up to an altitude of 3,600 m above sea level and its maximum airspeed is $148 \mathrm{~km} / \mathrm{h}$. SIERRA has been used in a number of environmental and geological studies, including the monitoring of floods, wildfires, volcanoes, mapping geological fault lines, and studying geothermal and other mineral resources [19].

UAVs have been used extensively to map and monitor the extent, biomass, and health of tidal wetlands, forests, agricultural crops, and other vegetation cover. Using specific types of imagers, they can map and classify coastal vegetation types using just a camera, GPS, and an inertial measurement unit (IMU) for reckoning the orientation of the UAV in 3D space. One example of this is shown by a fixed-wing UAV and a helicopter working together in remote rangelands to locate and spray weeds with herbicides. Another practical application of UAVs has been surveying of estuaries for harmful algal blooms.

The Kahu Hawk UAV used object-based image analysis (OBIA) to characterize the spatial distribution of the Newnes Plateau swamplands near Sydney, Australia, which was crucial in the identification of long-term changes in swamp conditions. The Kahu Hawk was a small fixed wing electric-powered aircraft weighing about $4 \mathrm{~kg}$ with a wingspan of $2 \mathrm{~m}$ and could either be controlled manually or with a flightpath uploaded to the autopilot prior to takeoff. Imagery data was collected at $121 \mathrm{~m}$ above ground level covering an area of about 26 ha [19]. The OBIA image segmentation method uses a computationally intensive software program to combine adjacent image pixels into distinct spatial objects. The segmentation algorithm is an automated process that is determined by user supplied control variables to create recognizable objects which are later assigned to semantic map classes (e.g. water, bare soil, or different vegetation types) [20]. This study also used NDVI to separate vegetation from non-vegetation pixels by assigning all NDVI values above zero as value ' 1 ' and all others as value ' 0 '. Using the 
binary NDVI as a mask to split the image into vegetation and non-vegetation, the space and time complexity of the OBIA classification process can be reduced. "This study demonstrated that images from low-altitude UAVs and OBIA image analysis methods can be combined to accurately classify vegetation extents" [19].

Another low-cost, practical UAV application of NDVI can be achieved through the use kites and balloons. "Kite and balloon aerial photography has been used for scientific surveys, meteorological observations, and military surveillance for centuries" [19]. A study done in Alaska in 2003 obtained high resolution images from a camera suspended from a kite. These images were then used to map water and vegetation as well as to perform geometric analysis of ice-wedge networks [19]. For light to moderate winds, rigid Delta kites are used. However in moderate to strong winds, parafoil kites are flown. Typical payload weights are in the range of one to five pounds and the size of the kite is dependent on wind conditions. "Home-made kits with attached camera rigs have also been used in archeology and in some developing countries to map local vegetation, obtain population counts in villages, and assess complex humanitarian emergencies" [19].

High-altitude balloons come in various sizes and shapes and can be used to not only measure atmospheric properties across a range of altitudes but also to monitor environmental changes at specific sites. "A tethered balloon can provide an excellent platform for imaging and tracking coastal dynamics, such as monitoring sediment transport over an oyster bed for several tidal cycles" [19]. A study done in 2012 by Shaw et al. [22] used tethered balloons to deploy a low-cost, compact, multispectral imaging systems in the blue, red, and NIR spectral bands at an altitude of approximately $300 \mathrm{ft}$. Vegetation was then identified and monitored primarily with the red and NIR bands for NDVI analysis whereas the blue band were used for analyzing water properties such as turbidity [19].

An innovative yet still inexpensive balloon configuration has recently been developed by the University of Delaware which combines a helium balloon with a kite. This balloon configuration is called the Helikite Aerial Platform (HAP) and is a multi-altitude, high spatial resolution system, which can fly up ton an altitude of $1.2 \mathrm{~km}$ and has a payload capacity of up to $5 \mathrm{~kg}$ [19]. The balloon has a diameter of about $3 \mathrm{~m}$, but it does not bounce around in the wind because it works like a kite with a constant lifting force that keeps extra tension on the tether line and provides added stability to the UAV. The HAP can be easily modified to carry various other types of cameras and payload, but most importantly it can be deployed rapidly and easily. The HAP has been used to obtain continuous images of river and coastal plumes with very high spatial and temporal resolutions. These images are then analyzed to study wetland changes and the evolution of coastal plumes, which have an important role in the mixing between high-nutrient terrestrial plumes and salt water. "Different designs of helikites have been used by other researchers to study relatively small sites, including submerged aquatic vegetation" [19]. 
As with the helium balloon, the blimp airship is also commercially available in various types for small format aerial photography. A typical blimp, shown in Figure 5, is constructed with single layer urethane and retains helium well with adequate durability for field conditions. It measures about $4 \mathrm{~m}$ long with a diameter slightly smaller than the length and has a payload capacity of $2 \mathrm{~kg}$. A helium-filled blimp has been deployed from a vessel during oil recovery operations and used to transmit images to an onboard user station that displayed images in real-time. "The application of this technique increases oil recovery rates and produces savings, especially if dispersants are used on the oil" [19]. Flying at an altitude of $150 \mathrm{~m}$, the field view of the camera was a little over $6 \mathrm{~km}$. The blimp was deployed and recovered using a winch to reel the tether out and back [19].

UAVs, or drones, offer a feasible alternative to conventional remote sensing platforms for obtaining high-resolution data at a reduced cost, with better operational flexibility, and with greater versatility. Quadcopter have a significant advantage over fixedwing aircraft simply because of their ability to hover and loiter over a target site and flexibility to change altitude at the same site for mapping at preferred spatial resolutions [19]. "UAVs have the capability to effectively fill current observation gaps in environmental remote sensing and provide critical information needed for coastal change research, wetland mapping, water resources forecasting, ecosystem monitoring, natural hazard prediction, and damage assessment. UAVs expand our ability to observe dynamic landscape-altering events and to conduct surveys in logistically challenging areas" [19]. A typical quadcopter UAV with an integrated high-definition camera is illustrated in Figure 15.

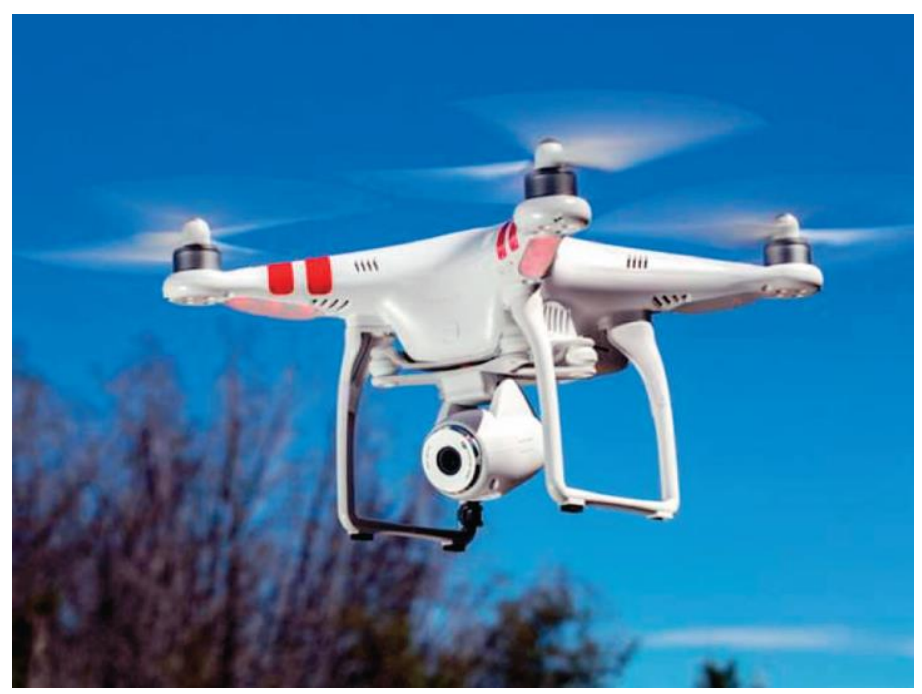

Figure 15: Typical Quadcopter with Integrated Camera [19] 


\subsection{Space Applications of NDVI}

\subsubsection{MODIS}

The MODIS sensor suite aboard the NASA Terra and Aqua satellites has proven to significantly improve the quality and depth of scientific studies performed around the world. Launched in December of 1999 as the flagship of NASA's Earth Observing System (EOS), the Terra satellite's mission was to answer the questions of how Earth is changing and what the impacts are for life on Earth [28]. A few years later in May of 2002, Terra's sister satellite Aqua, was launched into orbit. The Aqua satellite shares some identical sensor suites with Terra, including MODIS, while other science packages aboard Aqua are specifically designed for studying the water cycle. Aqua was the first of series of satellites that formed the "Afternoon Constellation" a.k.a. the "A-train" [29]. An illustration of the various Earth Science missions comprising NASA's Earth Science Division are shown in Figure 16 with corresponding launch years.

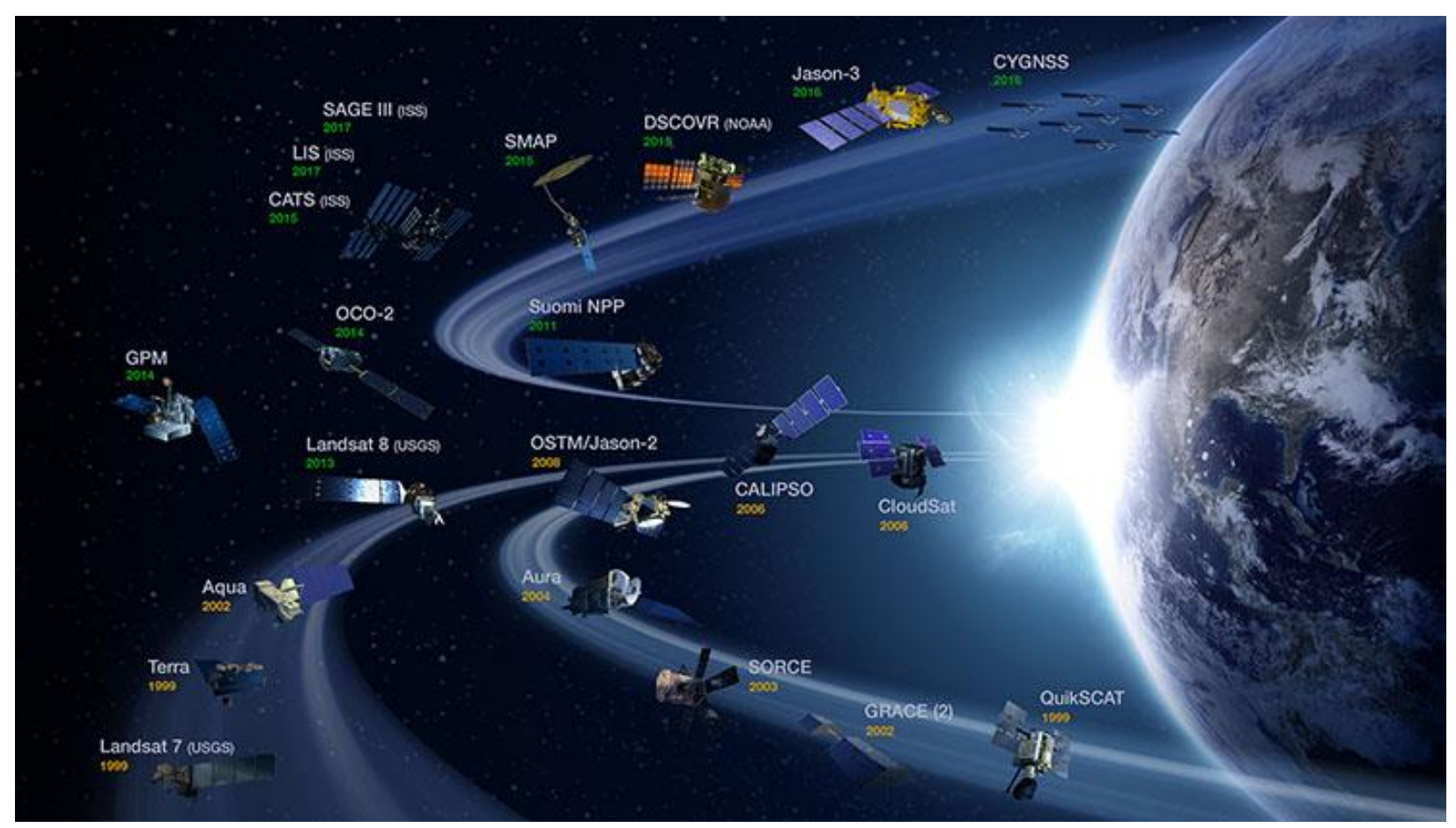

Figure 16: NASA Earth Science Division Operating Missions [30]

Since the 1980's, crop yields in many countries were predicted using NDVI data derived from the National Oceanic and Atmospheric Administration (NOAA) Advanced Very High Resolution Radiometer (AVHRR). MODIS NDVI data from 2000 to present as allowed significant improvements that address the shortcomings of AVHRR. For instance, the estimation of crop yielding is vital to food security. "Normalized Difference Vegetation Index (NDVI), as an effective crop monitoring tool, is extensively used in crop yield estimation" [31]. Winter 
wheat production in Shandong Providence, China, was estimated using spatial accumulative and smoothed MODIS-NDVI data. Meanwhile, an empirical approach for winter wheat production in Kansas and Ukraine was established using MODIS satellite data. Sugarcane yields in Kenya were predicted by applying weighted temporal series NDVI data from MODIS [31].

A recent study performed in the Nanpan River Basin located in Yunnan Province, China established a relationship between crop yield and NDVI data from MODIS. Crop estimation models are established based on crop classification and extracted NDVI data from crop planting areas. This approach was applied to three counties of Yunnan (Jianshui, Luliang, and Qiubei) and focused on three main crops: paddy rice, winter wheat, and corn. An initial study attempted to find a simple method for crop yield estimation by correlating crop yield for every 16-day period of NDVI data from MODIS. "However, no acceptable or reasonable result was found from the direct correlation analysis. Moreover, a higher correlation coefficient was obtained with the NDVI integrated over several periods" [31]. In fact, the results were better than relating the averaged NDVI during whole crop growth stages to crop yield because some periods are more important for crop yield, e.g. the heading to flowering stages for winter wheat. In practice, many types of crops are grown together in small regions. However, extracted NDVI data from corresponding crop planting relate directly to crop yield. "Therefore, identification of the crop planting area is another crucial part of the estimation model" [31]. In addition to crop planting areas, the best periods for a reliable estimation have been identified based on the correlation analysis between MODIS-NDVI data and crop yield. According to the Nanpan River Basin study, the best time coincides with periods of heading, flowering, and filling of crops. With mean NDVI data extracted from corresponding areas, this method for estimating crop yield seems practical and reasonable [31].

Another study that focused on the western United States in the mid 2000's used MODIS NDVI data to analyze evergreen die-off [32]. The overall goal of the analysis was to identify areas experiencing vegetative stress and resulting mortality using NDVI generated from seven years of Terra MODIS imagery data (2000-2006) and to evaluate the effects of environmental variables on the severity of die-off at local and regional scales. It has been shown that many factors including drought induced water deficit can lead to vegetation stress or mortality. "Recent fluctuations in global climate have had profound effects on the semi-arid ecosystems of western North America" [32]. The MODIS sensor suite was used to calculate NDVI during the North American growing season from 2000-2006 in several locations across the western US. Multispectral satellite images clearly show an identifiable reduction of cover and reduced health in these areas [32].

In addition to NDVI, there are several families of vegetation indices used to analyze vegetative health with remotely-sensed data at different spatial and spectral resolutions, and in different vegetation densities and atmospheric conditions. Some other methods include, but are not limited to, the Enhanced Vegetation Index (EVI), the Normalized Difference Red Edge (NDRE), and Soil Adjusted Vegetation Index (SAVI). "NDVI has been shown to be one of the better vegetation indices to determine total photosynthetic activity within moderately to sparely 
vegetated areas, and the bands required to generate NDVI or actual preprocessed NDVI product are readily available for download for little to no cost from multiple sources" [32].

NDVI has been shown to be a robust method for analyzing plant health for multiple applications. "The strength of NDVI lies in its ratioing concept, which reduces many forms of multiplicative noise (illumination differences, cloud shadows, atmospheric attenuation, and certain topographical variations)" [32]. A logarithmic stretch of the simple ratio is approximated by NDVI which allows for low biomass vegetation conditions to span across a wider range values compared to the simple ratio. Equation (2) defines the simple ratio $S R$ of the NIR and VIS parameters while Equation (3) describes the derived relationship between NDVI and $S R$ when Equation (2) is substituted into Equation (1):

$$
\begin{gathered}
S R=\frac{N I R}{V I S}, \\
N D V I=\frac{S R-1}{S R+1},
\end{gathered}
$$

NDVI values can vary from -1.0 to +1.0 with greener surfaces indicated by high positive values closer to 1, where NIR > VIS [32]. Water stressed or dead vegetation reflects less NIR and more VIS resulting in much lower NDVI values. This makes sense because heathier green vegetation absorbs more visible light for use in photosynthesis while reflecting large amounts of nearinfrared light. "NDVI is responsive to the health of green vegetation and can be used to monitor stress long before the vegetation loses its greenness" [32].

An average NDVI image for the 2006 growing season was composed and then subtracted from the average NDVI image for the 2000 growing season. Change in NDVI values, or delta NDVI values, were divided into three categories representing degree of die-off. The resulting image shown in Figure 17 illustrates the greatest amount of die-off of vegetation within the study period. Die-off ranges (i.e. changes in NDVI value) from 0-7\% are defined as MILD, 7-20\% are defined as MODERATE, and $>20 \%$ are defined as EXTREME [32]. Plant health improvement is represented by negative pixel values (i.e. delta NDVI values < 0). The areas of interest (AOIs) for the western US study are outlined by the numbered rectangles in Figure 17 and were chosen because they represented the most intense examples of severe die-off for those respective regions. The most negative values are represented by the areas shown in white which indicate areas of growth. The areas shown in black are areas with the highest value of delta $(\Delta)$ NDVI which is interpreted as die-off or plant stress. Based on in-field verification testing, MILD die-off pixels revealed up to $10 \%$ mortality, whereas MODERATE die-off pixels revealed between $10 \%$ and $70 \%$ mortality, and EXTREME die-off pixels revealed $>70 \%$ mortality [32]. The true dieoff results from plant water stress in the western US study had to be differentiated from forest fires. "Geospatial layers for forest fires were obtained and overlaid on the $\triangle$ NDVI images to determine where forest fires had occurred in relation to the $\Delta$ NDVI severity. In nearly all cases, 
areas having evergreen forest cover, and showing EXTREME (>30\%) die-off, corresponded with forest fires as catalogued by the United States Department of Agriculture Forest Service" [32].

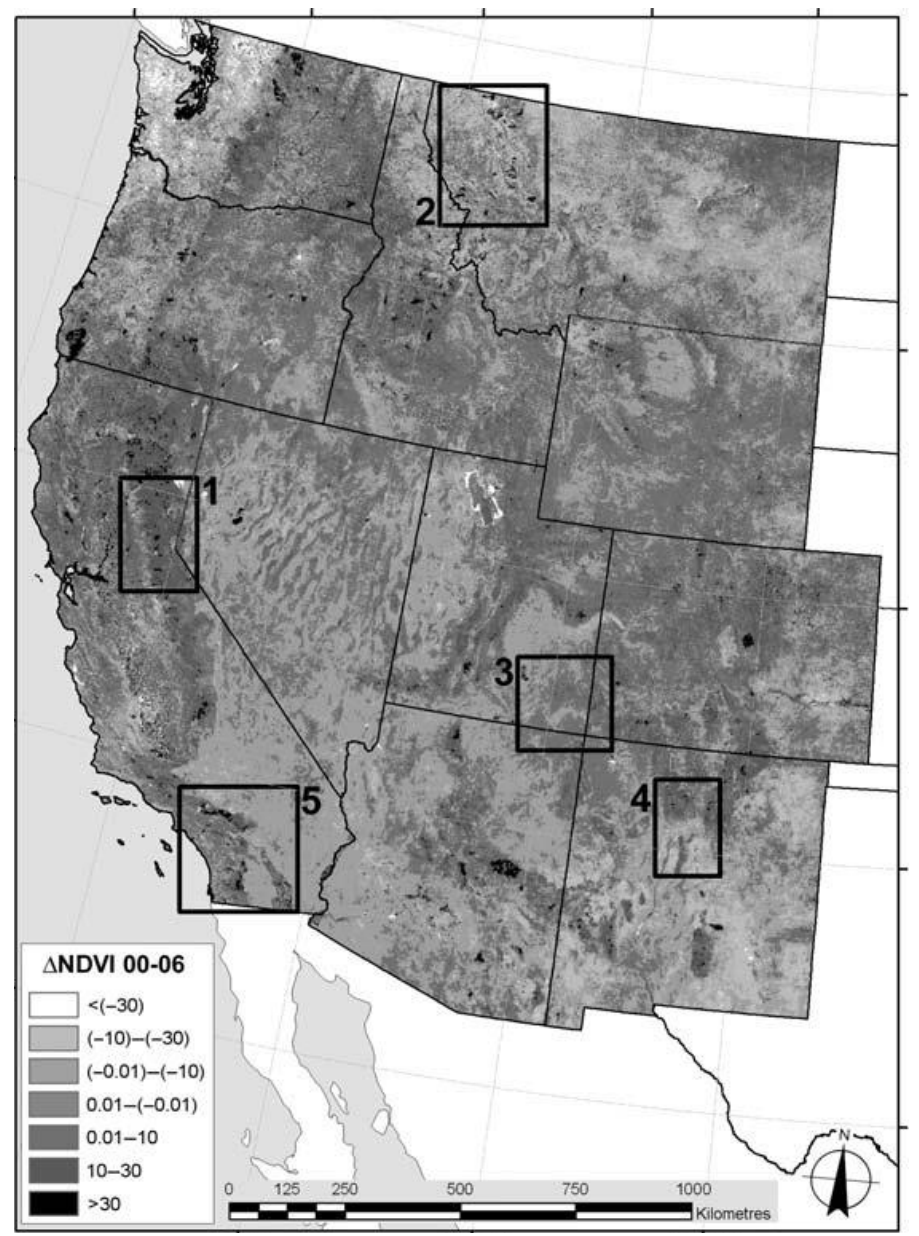

Figure 17: 2000-2006 Cumulative Change in Growing Season NDVI for the Western United States

MODIS data was also used in a research study performed on wildfire propagation in the Mongolia-China grasslands and the effects of live and dead fuel [9]. Data was gathered from 2001 to 2007 during the wildfire seasons of April to May and September to October. The goal of this study was to determine which areas would be most affected by wildfires by evaluating the susceptibility of wildfire propagation in the northeast Asian grasslands. Multivariable logistic regression was applied to MODIS data and used as a basis for the detection of burned areas and evaluation of fuel conditions. "Fuel is one of the most significant variables in fire ignition and behavior. Therefore its type, moisture level, and load must be carefully analyzed" [9]. Wildfire propagation and the resulting burnt areas are influenced by several factors, such as the location of the fire origin, topography, weather, fuel conditions, and active firefighting. In the Asian grassland study, burnt areas were the dependent variable whereas the MODIS-derived indicators and topographical factors served as the independent variables. Figure 18 shows the grasslands in north China and eastern Mongolia that were selected as the study area. This area covers 
approximately 1.40 million $\mathrm{km}^{2}$ and is characterized by cold and dry winters and warm and humid summers [9].

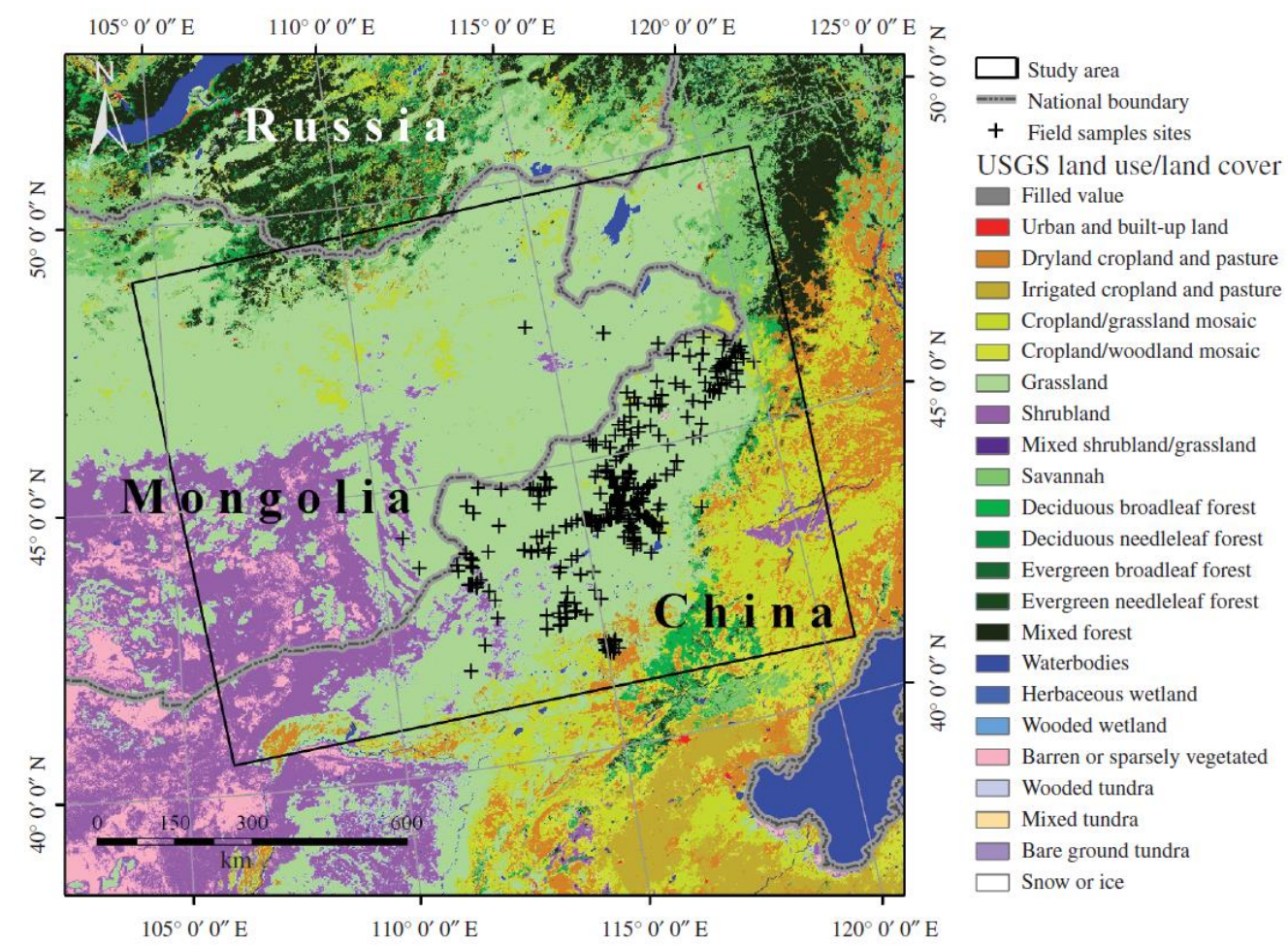

Figure 18: Asian Grassland Study Area with Land-use/Land-cover Types and Locations of Field Samples [9]

\subsubsection{Landsat}

Formerly known as the Landsat Data Continuity Mission (LCDM), the Landsat program's crucial role in providing data to help scientists and leaders understand and manage the necessary resources for sustainment of life is continued by the Landsat- 8 satellite which is NASA's eighth satellite in the Landsat series. With more than seven billion people living on the planet, Landsat monitors the changes in the environment and increasing impacts of human society [24]. For example, it has been shown that global forest loss has increased by approximately $2000 \mathrm{~km}^{2} /$ year between 2000 and 2012. A recent study performed in 2016 used Landsat time series (LTS) data from 2010 to 2013 to investigate deforestation in tropical areas of Brazil, Vietnam, and Ethiopia. These sites were chosen based on their differing ecosystem characteristics, frequency of observations, and deforestation types [6].

Previous developments of coarse spatial resolution systems, such as MODIS and AVHRR sensor suites, have inspired the application of time series analysis methods to Landsat data. "Compared to those missions, Landsat offers the longest running cross-calibrated globally consistent record of the Earth's surface at medium resolution" [6]. The main objective of the 
deforestation study was to identify which vegetation index (VI) performed the best in the detection of deforestation in the tropics when the Breaks For Additive Season and Trend (BFAST) Monitor algorithm is applied to LTS data. Emerging as a reliable tool, BFAST can be used to detect droughts, fires, changes in vegetation and forest landscapes, and other ecosystem disturbances. However, the ability of BFAST Monitor to describe the time series is dependent on the observation frequency and therefore can affect the ability to detect deforestation [6].

Eight commonly used VIs were used in the deforestation study, including NDVI which is the most frequently used VI in remote sensing science. "A search in Scopus revealed that more than 1000 peer reviewed journal articles use the NDVI to study tropical forests" [6]. NDVI makes use of strong reflective nature of vegetation to NIR light wavelengths and distinct differences in the reflection of visible light. This allows for monitoring photosynthetically active biomass. "This VI was successfully used in previous studies within a similar context" [6]. The BFAST Monitor was found to be robust in dealing with a significant amount of cloud residuals and related artifacts left in the data. A robust system for processing large quantities of remote sensing data called the Landsat Ecosystem Disturbance Adaptive Processing System (LEDAPS) was used to analyze forest change and was found to be a suitable pre-processing method for analysis of LTS data with the BFAST monitor method [6].

Another important parameter in agricultural and forestry applications is the vegetation water content (VWC). VWC analysis can provide information that can potentially drive irrigation decisions based on inferred water stress, aid in crop yield estimation, and assess drought conditions. The determination of fire susceptibility is a principle application of VWC to forestry. Research performed in 2003 established relationships between VWC and two remotely sensed indices, NDVI and the Normalized Difference Water Index (NDWI), using Landsat Thematic Mapper and Enhanced Thematic Mapper Plus data in conjunction with ground-based VWC measurements [12]. It was found that water content relationships vary by vegetation type and thus land cover was determined to be a significant factor when using NDVI and NDWI to estimate VWC. Useful VWC functions based on NDVI can be established when supported by ground sampling for specific canopy types within specific regions [6][12]. NDVI is a regular product typically produced every 10 days and made globally available using satellite instruments such as the AVHRR, which is advantage to using NDVI over NDWI for VWC estimation. However, NDVI is not responsive to the full range of canopy VWC because it produces saturated results at intermediate values of leaf area index (LAI). Figure 19 shows the relations established between NDVI and NDWI throughout the study period. For corn, NDVI values are saturated during the later period of the study while NDWI continues to increase until a maximum is reached. Based on a quantitative analysis of bias and standard error, NDWI was found to be a superior method for mapping VWC [12]. 


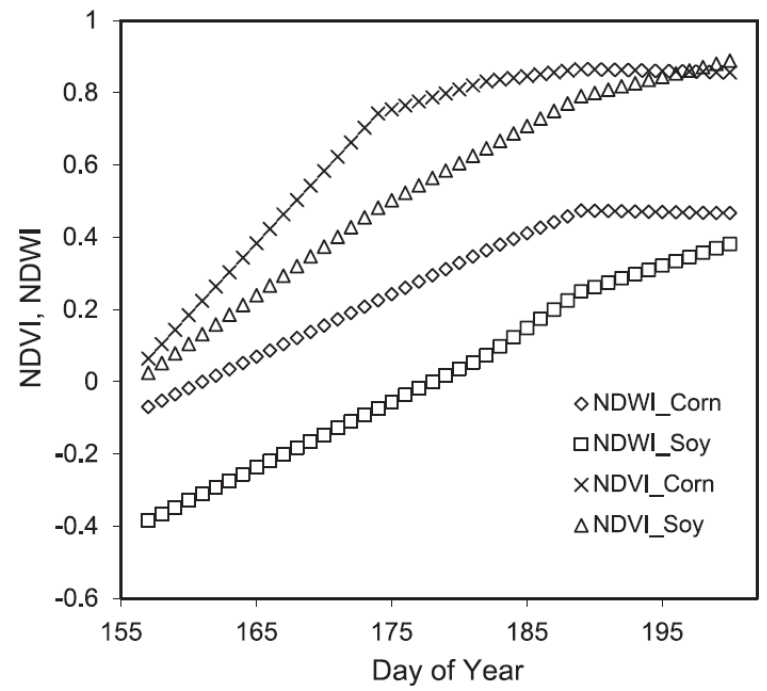

Figure 19: NDVI and NDWI Relationships for Corn and Soybean [12]

Land-cover mapping is currently an important research topic in studying land change and landscape planning. Land-Use and Land-Cover (LULC) maps are generated from high-resolution images to estimate urban sprawl and to plan transportation and infrastructures accordingly. These maps are also useful in the decision-making process for management of sustainable land resources. Classification approaches and techniques for detailed land mapping, urban planning, urban environment and ecology, urban heat island effects, vegetation managements, and support of research communities in land-change science all demand fine-scale land cover mapping which can be complex due to the heterogeneous nature of land systems. "Researchers are switching from traditional pixel-based methods to alternative approaches in image processing, namely the Object-Based Imagery Analysis (OBIA)" [33]. The OBIA method for mapping land cover uses advanced image segmentation techniques to group pixels into image objects which serve as its basic unit to avoid or minimize disturbance in the data within ground objects.

A study done in 2014 on a test site in Tippecanoe County, Indiana, used countywide aerial photo mosaics and a digital elevation model as inputs to OBIA algorithms to generate land cover mapping with relatively high accuracy. The National Land Cover Database (NLCD) data is produced from the Landsat Thematic Mapper (TM) and has 30-meter resolution. The NLCD is among the highest spatial resolution LULC data currently available that covers the US [33]. Principal component transformation was used to pre-process the satellite imagery data for reduced spectral dimensionality. Masks determined by NDVI were applied to separate vegetation and non-vegetation areas. Vegetation areas were further separated into three categories: tree, grass, and crop. These vegetation objects were delineated using their own distinct spatial and spectral features so that the boundaries of each vegetation type can be found [33]. Two segmentation algorithms were used in the Tippecanoe county study: multi-threshold (MT) and quadtree-based (QT). Figure 20 illustrates the results of both QT and MT segmentation on a satellite image taken in the PCA2 band. The original image is shown on the left while the 
resulting image after QT and MT segmentation is shown on the right. QT segmentation is used to split the images into a finer level of image object domains. Most of the large squares represent crops whereas the small squares represent trees. The blue dots indicate non-vegetation objects which are obtained by the results of MT segmentation [33].

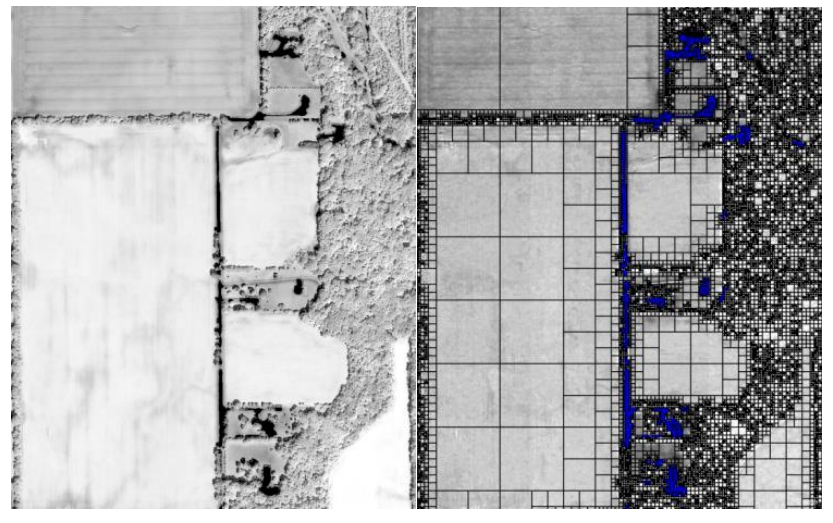

(a)

(b)

Figure 20: Image Segmentation Partition of Image into Objects (a) Raw Satellite Image with 800x1000 Pixels (b) Application of QT and MT Segmentation [33]

In terms of landscape planning, studying regional land-use and land-cover changes, and projecting environmental sustainability, the OBIA method provides a robust classification for fine resolution land-cover mapping. Three representative areas were identified using OBIA to demonstrate the benefit of using object-based methods. In pixel-based classification results, a "salt and pepper" effect/noise was observed as speckles in the image. Also, grass and tree classes were frequently confused with the crop class and buildings, road, and parking lots are easily misclassified as soil using the pixel-based approach [33]. The object-based approach generally resulted in more detailed land cover mapping when used to classify high-resolution aerial photography with ancillary elevation data. "The classification results show that this approach is more effective than pixel-based methods in terms of classification accuracy. While only spectral reflectance is considered in pixel-based approaches, the OBIA method utilizes multiple segmentation algorithms that help improve classification accuracy" [33]. It is possible that even more accurate land-cover maps may be generated using additional ancillary data, such as 1-meter resolution Light Detection and Ranging (LiDAR) data. [33]

\subsection{Cubesats for Earth Science}

Cubesats are regarded as a viable platform for future scientific missions and many surveys of nanosatellites and picosatellites, primarily Cubesats, are available in the literature. Traditionally, Cubesat missions have been educational development efforts with many of them used as technology demonstrators. These types of missions usually do not have stringent 
scientific requirements. However, it is common for Earth science instruments to be flown on Cubesats as either primary or secondary payloads. "A large number of Cubesats with some Earth observation capability have been developed in the last years, although most of them carried space weather sensors or modest resolution optical cameras" [26].

Since the development efforts of Cubesat missions are typically done on a much smaller budget compared to larger satellites, the payload design frequently incorporates COTS components with the use of open-source software and modeling tools where possible to reduce costs. Using COTS microcontrollers on Cubesats allows for higher data processing capabilities compared to space qualified components. However, there is a higher risk for susceptibility to space radiation since COTS microcontrollers typically are not manufactured with radiation hardened electrical components and materials. As of 2011, the standard bus system in Cubesat missions was I2C, despite the sporadic use of USB and CAN buses [26]. Typical on-board data storage systems for Cubesats range between $32 \mathrm{kB}$ and $8 \mathrm{MB}$, though additional flash memory of up to 8 GB has been employed in some payload designs. For a VGA camera with a 640 x 480 pixel resolution, 8 bit/pixel and no data compression, $8 \mathrm{~GB}$ equates to about 3500 images. However, the data storage capacity is irrelevant if higher data rates cannot be achieved. "In fact, it is trivial to show that there is a linear relationship between storage capacity and data rate if the constraint is enforced to have enough storage capability to store all the images that can be downloaded in one access to the ground station"[26]. This relationship is shown in Equation (4):

$$
\text { Storage }(M B)=\frac{15}{2} \Delta T\left(\frac{\min }{\text { access }}\right) R_{b}(\text { Mbps })
$$

where Storage $(M B)$ is the storage in megabytes required for the memory dump, $\Delta T$ ( $\mathrm{min} /$ access) is the average duration of a communication access to the ground station in minutes, $R_{b}$ is the downlink data rate in megabits per second, and the factor 15/2 (or 60/8) comes from transforming minutes to seconds and bits to bytes as shown in Equation (4) [26]. Returning to the example of the VGA camera, a typical image file size is 2.34 Mbit. The number of images, $N$, capable of being downloaded per flyover is driven by the access time and data rate by Equation (5)[26]:

$$
N(\text { image } / \text { access })=\frac{60 \cdot \Delta T(\mathrm{~min} / \text { access }) R_{b}(\text { Mbps })}{S_{\text {img }}(\text { Mb/image })}
$$

where $S_{\text {img }}$ is the size of each image in Megabits. This results in a total of 64 images when an average access time of 5 minutes and a data rate of $0.5 \mathrm{Mbps}$ are again assumed. The amount of data is relatively limited compared to larger satellites and is emphasized when the lifespan of the Cubesat is considered, which is on the order of 1 year compared to 5-10 years or more for higher class missions [26]. 
Spectral assessments of vegetation state and biomass using NDVI have perhaps the longest heritage of vegetation measurement. NDVI measurements were typically done with low spectral resolution radiometers such as Landsat/TM and NOAA/AVHRR. "The next generation of vegetation instruments improved almost an order of magnitude in spectral resolution with MODIS" [26]. The Can-X2 Cubesat carries a payload that achieves the required spectral and spatial resolutions for NDVI measurements similar to the Landsat/TM and NOAA/AVHRR. "Similar techniques have been demonstrated in Unmanned Aerial Vehicles (UAV) as well. Higher performance vegetation measurements (e.g. MODIS) including sensible imaging and hyperspectral capabilities are more difficult to achieve in a cubesat-based mission, due to the trade-off between number of channels, spectral resolution, and swath in a very confined space" [26]. A preliminary assessment of cubesat-based mission utility for measuring various land, ocean, and atmospheric parameters is provided in Appendix A.

Science education and technology utilization in emerging and developing regions can be promoted through cubesat development, operation and data analysis. The small, lightweight cubesat platform offers inexpensive construction and affordable launch opportunities through standardized development kits and 'piggyback' rides with larger satellites traveling to low-earth orbit (LEO), Moon, or even Mars. It is envisioned that the future large-scale science missions will use constellations of cubesats to achieve their observational objectives [34].

"Universities pioneered the development of the smallest of the small satellites - the "nano" and "pico" categories to which cubesats belong" [34]. Conventional development of larger satellites typically requires multi-year development and large professional teams, making it capital and expertise intensive. Thus, participation of science and engineering students in conventional satellite development is drastically limited. Jordi Puig-Suari and Bob Twiggs realized this problem and found it particularly problematic for aerospace engineering students, which inspired them to introduce the cubesat specification of a $10 \mathrm{~cm}$ cube with a mass of up to $1 \mathrm{~kg}$. An example of a standard cubesat platform is shown partially assembled in Figure 21.

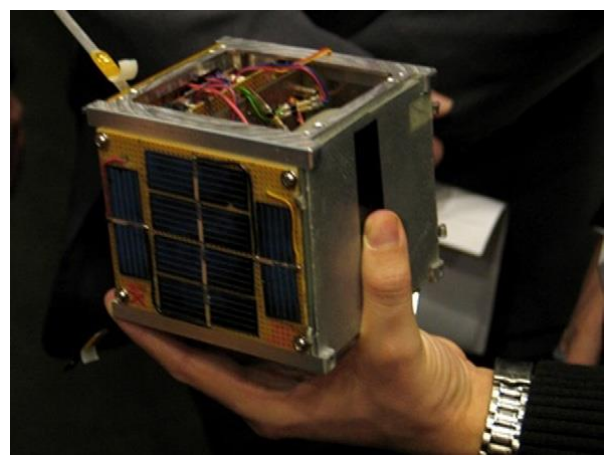

Figure 21: Standard Cubesat Platform [26]

"Smallsats including cubesats have spawned significant commercial activity, including providers of complete satellites, components, and launch services, many of them starting as academic spin-offs" [34]. Public-domain access to the Cubesat Standard coupled with the 
availability of graduates with education and experience on the cubesat platform, has undoubtedly furthered its commercial success. An early example of an academic spin-off success story is the collaboration between Pumpkin, Inc. and Stanford University leading to the development of the Pumpkin Cubesat Kit [34]. A list of reputable cubesat startup companies and component/service providers existing in 2011 are listed in Table 2. Today, the number of startup companies and providers has grown to over 200. As a result, there have been more than 850 nanosatellites launched into space and over 500 are currently still in orbit [35]. A bar graph showing the number of nanosatellites launched to date and projected launches within the next five years can be referenced in Appendix B along with a pie chart showing the current number of nanosatellites launched by each country.

Table 2: Cubesat Startup Companies and Component/Service Providers [34]

\begin{tabular}{|c|c|c|}
\hline Company & Products and/or services & Date founded, location \\
\hline Surrey Space, Ltd. & Small satellites & 1985, UK \\
\hline Tethers Unlimited & Tether technologies for orbital formation flying & 1994, US \\
\hline SpaceQuest, Inc. & Cubesat components & 1994, US \\
\hline Pumpkin, Inc. & Cubesat kits and integration services & 1995, US \\
\hline Sinclair Interplanetary & Attitude determination \& control for smallsats & 2001, Canada \\
\hline MicroSpace & MEMS microthrusters & 2002, Italy \\
\hline Dobson Space Telescope GbR & Telescopes and imagers for small satellites & 2002, Germany \\
\hline Clyde Space & Cubesat Kit components and design services & 2005, UK \\
\hline TriSept Corp. & Small satellite launch integration services & 2006, US \\
\hline ISIS & Small satellites and launch integration services & 2006, The Netherlands \\
\hline GOMSpace & Small satellites & 2007, Denmark \\
\hline
\end{tabular}

Cubesat-class spacecraft specifications have been designed to be compatible with a standardized launch vehicle interface called the Poly-Picosatellite Orbital Deployer (P-POD). With dimensions approximately $13 \mathrm{~cm}$ x $16 \mathrm{~cm}$ x $40 \mathrm{~cm}$, the P-POD can carry between one and three cubesats with a total mass of no more than $5 \mathrm{~kg}$. The standard cubesat unit size is $1 \mathrm{U}$, or 10 $\mathrm{cm} \times 10 \mathrm{~cm} \times 11 \mathrm{~cm}$. Thus, a total of $3 \mathrm{U}$ standard cubesat units can fit inside a P-POD. With respect to the launch provider, the P-POD essentially becomes a 'black-box' where the contents are not of importance to them as long as it contains the proper mass and maintains structural integrity throughout the launch [36]. The value this decouple provides to cubesat developers is huge and is the fundamental reason there seems to be an increasing number of 'piggy-back' launch opportunities being offered by government and commercial providers.

A research investigation on the first 100 cubesat development efforts was performed by Swartwout in 2013 at Saint Louis University. Only missions possessing an official launch manifest were considered as part of the first 100 cubesats. There were four categories of cubesat missions given to each based on the payload type and level of sophistication. "A T-class (technology-class) mission flight-tests a component or subsystem that is new to the satellite industry (not just new to the mission provider). An S-class (science-class) mission creates science data relevant to that particular field of study (including remote sensing). A C-class (communications-class) mission provides communications services to some part of the world (often in the Amateur radio service). Missions listed as E-class (education-class) lack any of the 
other payloads and serve mainly to train students/young engineers and improve the satellitebuilding capabilities of that particular program; typical E-class payloads include COTS imagers (low-resolution Earth imagery), on-board telemetry, and beacon communications" [36]. E-class cubesats are sometimes informally referred as "BeepSats", when they serve no on-orbit function other than continually sending back telemetry in regular intervals.

Figure 22 illustrates a breakdown of the four classifications of cubesat mission types investigated from 2000-2012. E-class and T-class missions seem fairly equal in total percentage breakdown while science missions make up roughly a quarter of the whole distribution. It makes sense that C-class missions are less common because communication satellites are typically placed in geostationary or Molniya orbits that are more expensive to reach compared to launches into LEO and to the International Space Station (ISS). Also, communication satellites are typically deployed in constellations of multiple spacecraft, which imply higher development costs for a single developer to build multiple cubesats in able to achieve a more comprehensive communication objective.

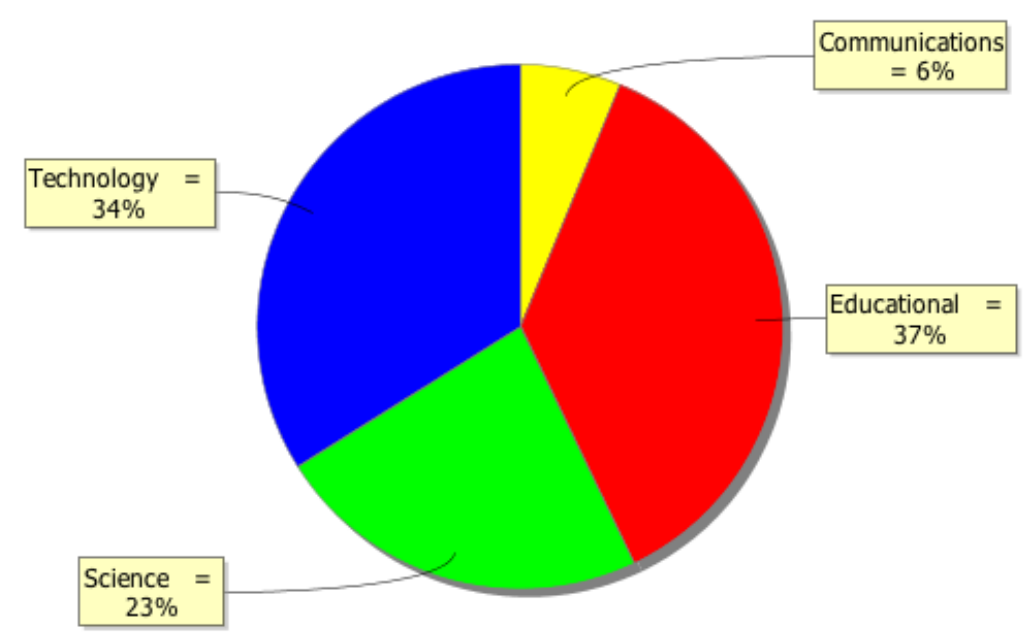

Figure 22: Cubesat Mission Breakdown by Mission Category, 2000-2012 [36]

Figure 23 shows the distribution of cubesat missions launched each year from 2000-2013, which included firm launch manifests for 2013. The color distribution represents the number of cubesats deployed per launch vehicle. It can be seen that a drastic increase was seen from 2012 to 2013 and the trend has continued to rise on an even greater scale (Appendix B) [35][36]. It has been predicted that the average number of cubesat deployments per launch will also rise. The U.S. will fly between 3-16 P-PODs through NASA's Educational Launch of Nanosatellites (ELaNa), mostly on government procured launches. However, with commercial launch providers on the rise this may no longer be the case in the not too distant future. 


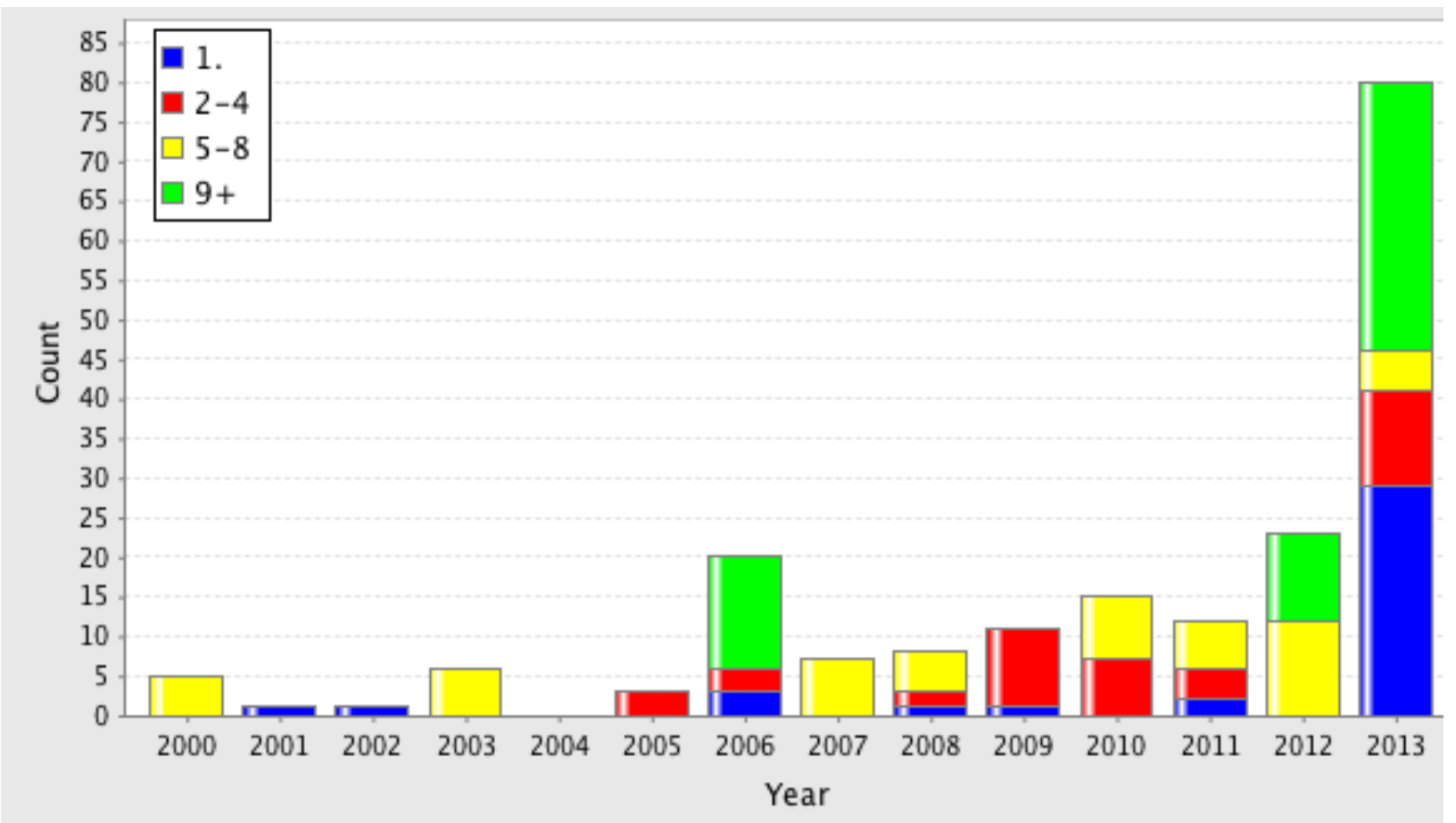

Figure 23: Number of Cubesats Launched Per Year and Per Launch Vehicle from 2000-2013 [36]

"The motivation for moving to 3U-sized spacecraft is a need for more volume and aperture; practically speaking, a $3 \mathrm{U}$ spacecraft has more than triple the power, communication, and pointing capacity of a $1 \mathrm{U}$ spacecraft. It is instructive to note that the very first $3 \mathrm{U}$ spacecraft, QuakeSat, was also the first successful S-class Cubesat, and that 28 of 30 3U-class spacecraft have had real missions (compared with 33 of $761 \mathrm{U}$ missions)" [36]. Five to six years ago, it could be assumed that a student-built Cubesat would be an E-class 1U, whereas an industry-built Cubesat would be a T-class 3U. However, the 2012 manifest marks a turn from 28\% (13 of 35) of all university-built $1 \mathrm{U}$ missions being S/T/C-class to nearly half (6 of 15). This could be due to the NASA ELaNa program and other launch initiatives. Moreover, traditional developments have shown that professionally-led Cubesat missions are almost always "real" missions of the S/T/C-class whereas university-led Cubesat missions are typically on the level of "BeepSats". "However, the recent trend among universities is to fly real missions" [36].

The Simulation to Flight 1 (STF-1) is the first Cubesat developed in West Virginia with a firm launch manifest and is being built as a collaborative effort between the NASA Independent Verification and Validation (IV\&V) Facility's Jon McBride Software Testing and Research (JSTAR) team and the West Virginia University (WVU) Statler College of Engineering and Mineral Resources (Mechanical \& Aerospace) and Eberly College of Arts and Sciences (Physics). "In February 2015, the STF-1 project was selected for participation in NASA's Cubesat Launch Initiative, meaning that the STF-1 team will get to create West Virginia's First Spacecraft! STF-1 will fly as a payload on the ElAnA XIX Electron, before being deployed at $500 \mathrm{~km}$ above Earth's surface" [37] [39]. 
There are four WVU space experiments flying on STF-1: Space Weather, III-V NitrideBased Materials, MEMS IMU Swarm, and Precise Orbit Determination. Various COTS parts are also employed in the STF-1, mainly assisting in power, communications, or computing [38]. "NASA's main goal in this initiative is to provide CubeSat developers access to a low-cost pathway to conduct research in the areas of science, exploration, technology development, education or operations" [40]. The main goal of the JSTAR team is to fully demonstrate the capabilities of the NASA Operational Simulation for Small Satellites $\left(\mathrm{NOS}^{3}\right)$ on the STF-1 mission. For the WVU space experiments, the main objectives were to demonstrate proof of concept or to prove functionality of sensors and devices. The Space Weather experiment incorporates a Langmuir probe for measurements of electron density and temperature, a radio sounder for collecting plasma density information and magnetic field readings, and particle counters for characterizing radiation intensity. The III-V Nitride-Based Materials experiment employs a precision optoelectronic sensor module to determine the optimal thickness for sufficient shielding from low temperatures and high levels of radiation while extending measurement capabilities. The MEMS IMU Swarm integrates 32 smaller, less expensive IMUs compared to high-end IMU devices with the concept that the results will be extremely accurate when averaged properly. Lastly, the Precise Orbit Determination uses a GPS receiver and GPS data received from various other satellites through NASA's GIPSY-OASIS technology to determine STF-1's exact location [38]. A picture of the fully assembled STF-1 3U spacecraft is presented in Figure 24. The fully-assembled mass was measured to be just below $3 \mathrm{~kg}$.

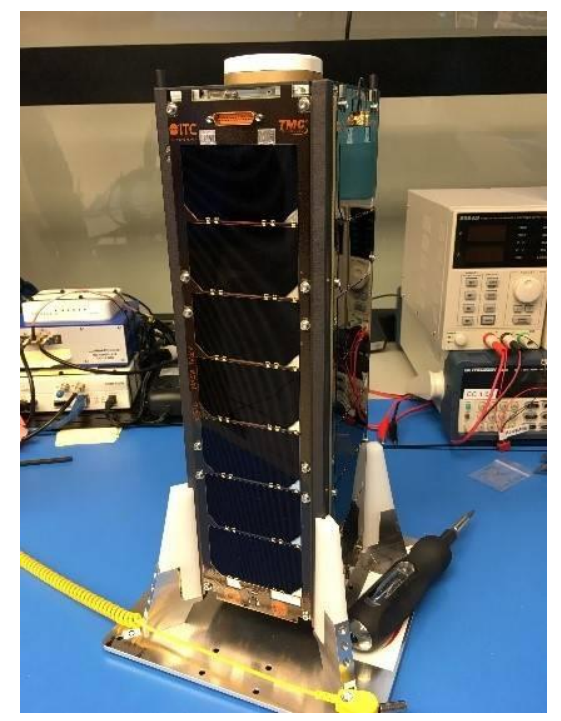

Figure 24: Fully-Assembled STF-1 Cubesat [40]

The NASA IV\&V Program has also started its own student outreach initiative known as NASA Space Flight Design Challenge (SFDC). Created in 2011, this initiative enhances critical systems education, tools and methods of verification and validation (V\&V), and software engineering approaches overall. The spirit of the challenge revolves around the use of COTS 
components to keep development costs at a minimum. Partnering with industry, academia, and other government agencies, the NASA SFDC initiative fosters innovative advancements in the application of IV\&V techniques whiles using hands-on development to enhance the knowledge and capabilities of the public. "Using an incremental approach, teams comprised of both college and high school students across West Virginia and eventually the nation will compete in building, testing, launching and operating flight systems in low Earth orbit through amateur radio operations" [41].

The first orbital mission to be flown under the SFDC initiative will be O'Conner Flight 1 (OCF-1). This mission is dedicated to former NASA Chief, Safety and Mission Assurance , Brian O'Conner, and serves to represent the "yes, if" attitude required to ensure mission success and to achieve ambitions goals set forth by the project [42]. The payload design incorporates science and engineering data measurements through the use of a relatively expensive 10 degreeof-freedom (DOF) IMU for tracking attitude and orientation, a highly sensitively 3-axis analog magnetometer, an OpenLog COTS micro SD card data logger device, and an Arduino microcontroller unit. A 3D mechanical model of the OFC-1 payload board is shown in Figure 25 with labels for the major components.

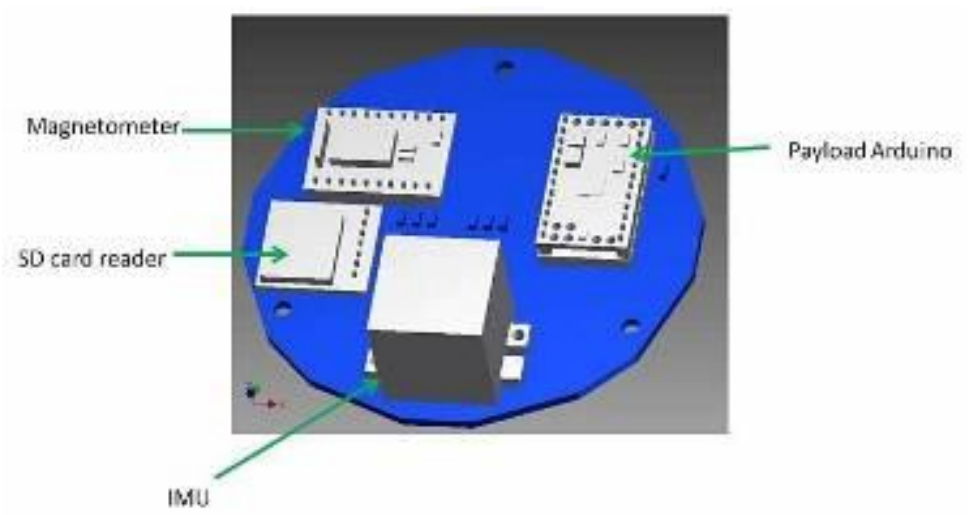

Figure 25: 3D Mechanical Model of the OCF-1 Payload Board [42]

The OFC-1 pico-satellite payload was designed to be compatible with the Interorbital Systems (IOS) Tubesat Kit [43]. A 3D mechanical model of the IOS Tubesat was exported to a rapid prototyping machine housed at the IV\&V Program's Educator Resources Center (ERC). The 3D model proved to be helpful for students working on the project to visualize the Tubesat as a full assembly while stacking the boards together in the proper configuration. A photo of the 3D printed model of the IOS Tubesat standing next to a stacked assembly of the IOS Tubesat Kit is shown on the left in Figure 26 along with a fully assembled Tubesat on the right. A mockup ejection cylinder is also present in the background. 


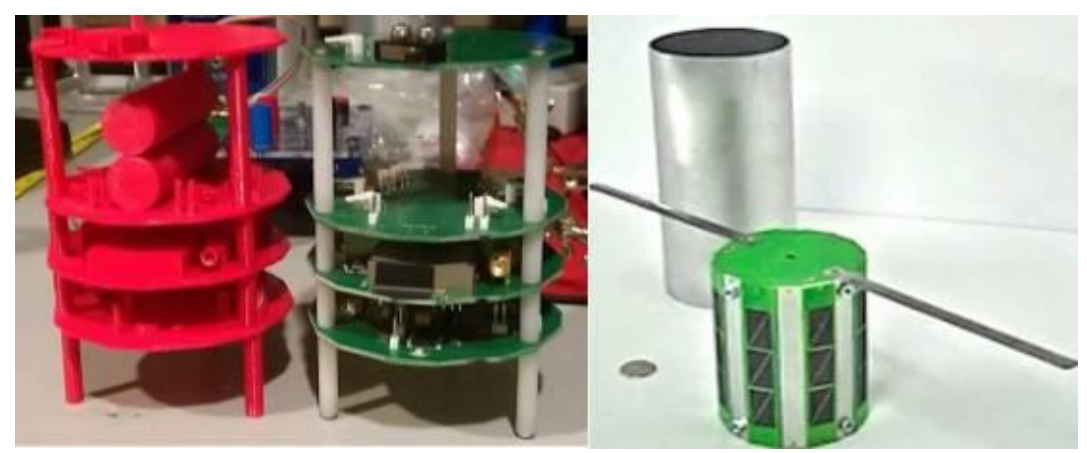

Figure 26: 3D Printed Model Next to Stacked Assembly of IOS TubeSat Kit (left) and a Fully Assembled Tubesat (right)

The OCF-1 spacecraft was built around the IOS Tubesat kit because IOS is also a lowcost launch provider which upholds the COTS objective of the SFDC initiative. The orbit profile provided by the IOS launch vehicle is a polar circular orbit with an inclination angle of $90^{\circ}$ at an altitude of $310 \mathrm{~km}$. With an orbital period of $\sim 90 \mathrm{~min}$, users can expect roughly 4 contact opportunities per day lasting around 8-14 min per flyover. The IOS Tubesat Kit includes printed circuit board (PCB) designs for the four major subsystems: Microcontroller, Communication, Power, and Antenna. Also included with the kit is an assembly guide, components list, basic hardware/fasteners, an Arduino microcontroller, $70 \mathrm{~cm}$ band transceiver, a $3.7 \mathrm{~V}$ lithiumpolymer battery, and 50 miniature solar cells [43]. It is the development team's responsibility to design the payload PCB for fabrication and to populate (i.e. solder) the electrical components on each PCB unless this service is purchased as part of PCB fabrication.

An IOS Cubesat Kit is also available, and the contents of which are very similar to the description of the IOS Tubesat Kit. "The CubeSat is a small satellite package optimized for containing small communications and experimental payloads into orbit. CubeSats can function as Basic Satellite Buses or simple stand-alone satellites" [44]. Developed in 1999, the IOS Cubesat Kit is capable of sending a signal from space that can be acquired on Earth with a handheld amateur radio receiver. The kit also provides a section to include experiments or other hardware as long as it fits within the volume constraints and has a mass of less than $10 \mathrm{~kg}$ total. Pictures of different version of the IOS Cubesat Kit are shown in Figure 27. On the left is a $1.3 \mathrm{U}$ Cubesat Kit and on the right is a 1 U IOS Cubest Kit [44]. The main difference is additional volume and mass available (i.e. $10 \mathrm{~mm}$ length and $1 \mathrm{~kg}$ for a $1 \mathrm{U}$ vs. $15 \mathrm{~mm}$ and $1.3 \mathrm{~kg}$ for a $1.3 \mathrm{U}$ ). 


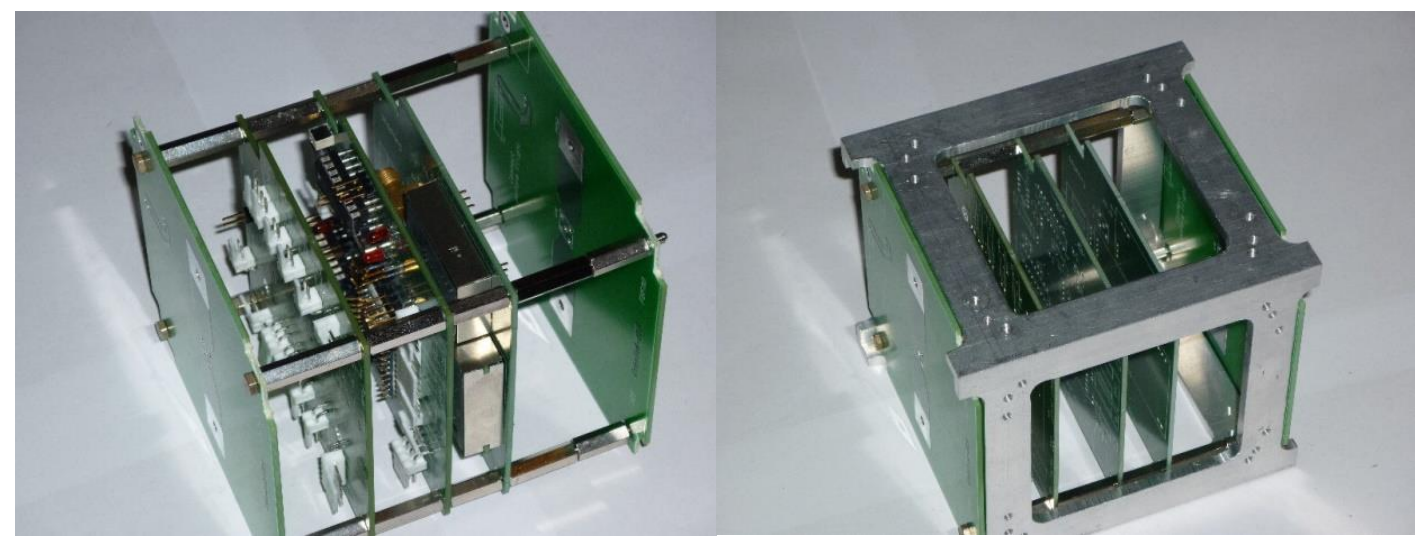

Figure 27: 1.3U IOS Cubesat Kit (left) and 1U IOS CubeSat Kit with Aluminum Structure (right) [44] 


\section{Chapter 3 | Experimental Design}

In this section, a description of the physical, electrical, and software design requirements is provided along with an overview of the system design. The hardware configuration settings adopted for this system are explained and a flowchart is provided to illustrate the software architecture and interactions within system. Finally, a picture of a portable prototype setup used for initial field testing is provided with details.

\subsection{Design Requirements}

\subsubsection{Physical Requirements}

The physical design requirements of the payload PCB were driven by the form factor of the IOS Cubesat Kit. The notches on each corner are necessary to fit securely inside the cubesat structure. It can be seen in Figure 28 that the lower left mounting hole is offset so that a reference can be established.

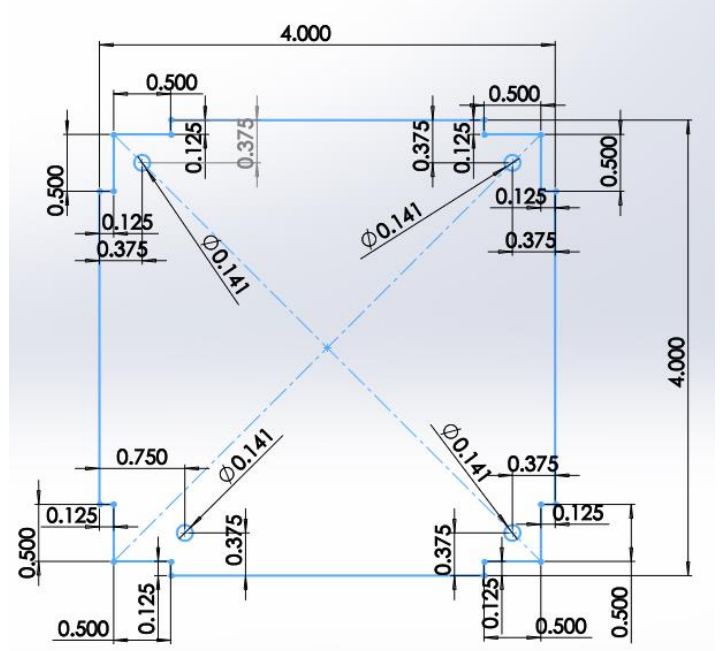

Figure 28: Design Dimensions of the Payload PCB

The mass requirements were also driven by the requirements for a cubesat which are set at $1 \mathrm{~kg}$. Since the IOS Cubesat Kit has a mass of about $700 \mathrm{~g}$, the maximum payload mass is $300 \mathrm{~g}$. The fully assembled payload weights $180 \mathrm{~g}$ with $120 \mathrm{~g}$ to spare. In addition to the mass requirement, another physical requirement is driven by the nature of the NDVI experiment. The two cameras used in the experiment must be positioned directly adjacent to each other such that the distance between the camera lenses is minimized. Finally, all wiring and harness connectors must be 'staked' or securely supported using wire ties and dabs of RTV silicon were necessary to reduce the risk of damage to wiring or loose connections occurring due to vibration. 


\subsubsection{Electrical Requirements}

Similar to the physical requirements, the electrical requirements were driven by the IOS Cubesat Kit which provides a $3.7 \mathrm{~V}$ single cell Li-ion battery. Therefore the input voltage was set at this value. There was also a hardware requirement for an input voltage of $5 \mathrm{~V}$ to power both Raspberry Pi (RPi) central processing units (CPUs). Thus, a derived requirement for a voltage regulator boost converter emerged. Since each RPi was expected to draw up to $750 \mathrm{~mA}$ during peak processing, the boost converter must output a maximum of $1.5 \mathrm{~A}$ or higher at $5 \mathrm{~V}$. With these requirements at hand, the Texas Instruments LM2731XMF switching boost converter chip was selected which offers a wide input voltage range of 2.7-14 $\mathrm{V}$ and a maximum output current of 1.6 A [44]. The power circuit configuration incorporated into the payload PCB design is shown in Figure 29.

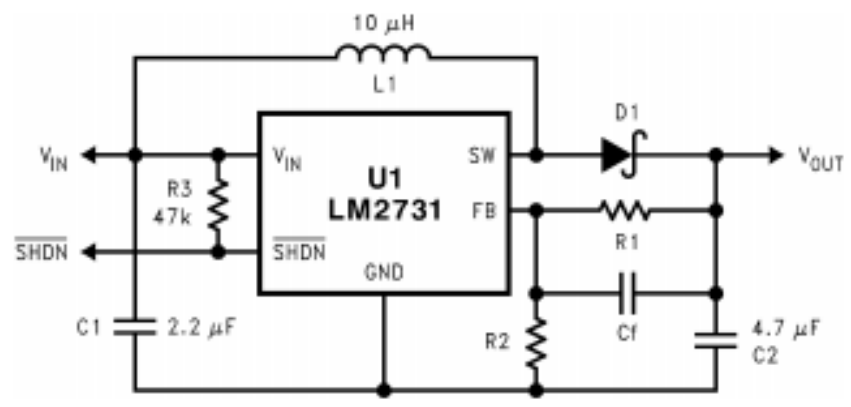

Figure 29: LM2731 Basic Application Circuit [45]

- The output voltage is set by the resistance value of external resistors R1 and R2. A value of $13.3 \mathrm{k} \Omega$ was used for R2 to establish a divider current of approximately $92 \mu \mathrm{A}$, as recommended by the manufacturer. R1 is calculated using Equation (6):

$$
R_{1}=R_{2}\left(V_{\text {out }} / 1.23-1\right)
$$

Another inherent power requirement was in the power connector which must not allow reverse polarity connection and should fasten the connector plug securely in place. The connector plug should also prevent the possibility of shorting the leads together. A standard 2pin JST connector was selected as the connector type in this payload design since to meet the requirements. Although it does not provide a locking mechanism, the plug does provide a keyway connection which prevents reverse polarity while still providing a secure, tight-fitting attachment. This was also the connector series that came installed on the battery from the manufacturer, therefore this reduced cost and effort by eliminating the need to purchase and replace a new female connector for the battery leads. Since the battery came installed with a female connector, this prevented any accidental shorting of the battery leads. 
The final electrical requirement was in camera connectivity. The system must allow for connection of two cameras such that image synchronization can occur. In other words, the system must provide a connection for the cameras and allow simultaneous image capture between two different cameras. The on-board camera connection was chosen as the design option for camera connectivity over a USB connection or I2C JPEG camera for multiple reasons. One reason is that the on-board ribbon cable connection allows for fast communication between the CPU and the camera device. Secondly, the low-cost USB web-cameras and JPEG cameras are typically low resolution and communication over USB is much slower resulting in much slower frame rates, whereas the latest version of the standard Raspberry Pi camera is $8 \mathrm{MP}$ which is fairly impressive for less than thirty dollars. Most importantly, however, a COTS No-IR camera is available from various vendors. This eliminates the need to modify a standard camera module for capturing NIR wavelengths, which could result in sensor contamination.

\subsubsection{Software Requirements}

In effort to uphold the spirit of the SFDC initiative for low-cost development, a Debianbased Linux open-source operating system called Raspbian was selected for use on the Raspberry Pi Zeros. This operating system was installed using the New Out of the Box Software (NOOBS) package provided by the Raspberry Pi open-source development project [46]. There was no room in the budget for purchasing proprietary software or hardware which provides software support. Therefore, the online open-source community for Raspberry Pi project development was relied upon for installation tutorials, programming examples, troubleshooting, and debugging software errors.

Perhaps the most critical software requirement of the NDVI payload system is in the capture of simultaneous images from the two different cameras. For still shots and stationary imagery, this requirement can be relaxed. The time in between image capture of the two different cameras is not as critical for still shots unless there are other external forces that cause changes in the scenery like wind or clouds passing by the sun. However, since the target use case for this payload design is intended to be UAVs, general aviation, and Cubesats, this requirement is crucial to maintain integrity of the NDVI imagery data obtained during flight.

Finally, a fault tolerance requirement was imposed to ensure that the software continued to function during a communication fault between the two RPi CPUs. In the event that the NIR camera connection is lost, the slave CPU should reboot. Similarly, if the RGB camera connection is lost, the master CPU should reboot. In the event that GPIO initialization error occurs on either CPU, the pins should be reset. If GPIO connection between the master and slave CPU become disconnected, a timeout sequence should execute which allows both the RGB and the NIR camera to continue to take pictures out of synchronization such that some useful data may potentially be extracted to achieve minimum success. 


\subsection{System Design}

The concept of using a single CPU connected to both NIR and RGB cameras was considered along with having a master/slave configuration with communication links to each other. Initial testing determined that the use of a multi-camera adapter module for expanding the number of camera ports on a single Raspberry Pi did not satisfy the requirement because a minimum of about a 5 second delay was observed between the switching of camera modules. Therefore, it was decided that the use of a master and slave RPi configuration was more feasible. This design decision then imposed a new derived requirement for the communication between separate CPUs to synchronize image capture. Several communication methods were considered including Ethernet, USB, and general-purpose input/output (GPIO) pins. Continued testing showed that Ethernet connections were less stable during reboot scenarios from unexpected power disconnection or battery depletion. In effort to reduce complexity, a simple flag approach was taken using general purpose input/output (GPIO) digital pins to synchronize the capture of images between the two different cameras.

The main components that comprise the proposed NDVI cubesat/UAV payload are the master and slave Raspberry Pi Zero CPUs along with the standard 8 Megapixel (MP) Pi-Cam version 2 RGB camera and the $8 \mathrm{MP}$ Pi No-IR version 2 NIR camera. The RPi Zero is the smallest single board computer offered in the RPi family with the exception of the Pi Compute module that requires an additional development board to develop a project and is significantly more expensive. Each RPi was installed with a 32 GB class 10 micro SD card which is more than enough for most applications. The RGB camera was connected to master RPi with a ribbon cable and likewise the NIR camera was connected to the slave RPi. A $3.7 \mathrm{~V}$ single cell 2500 mAh Lithium-polymer battery was used to power the experimental payload. Redundancy in the power connection was incorporated through the use of two separate $5 \mathrm{~V}$ power (PWR) pins and two separate ground (GND) pins. In case one of the wires becomes temporarily or permanently disconnected, the system will maintain power and not reboot or lose power indefinitely. A functional block diagram of the experimental NDVI payload is shown in Figure 30. 


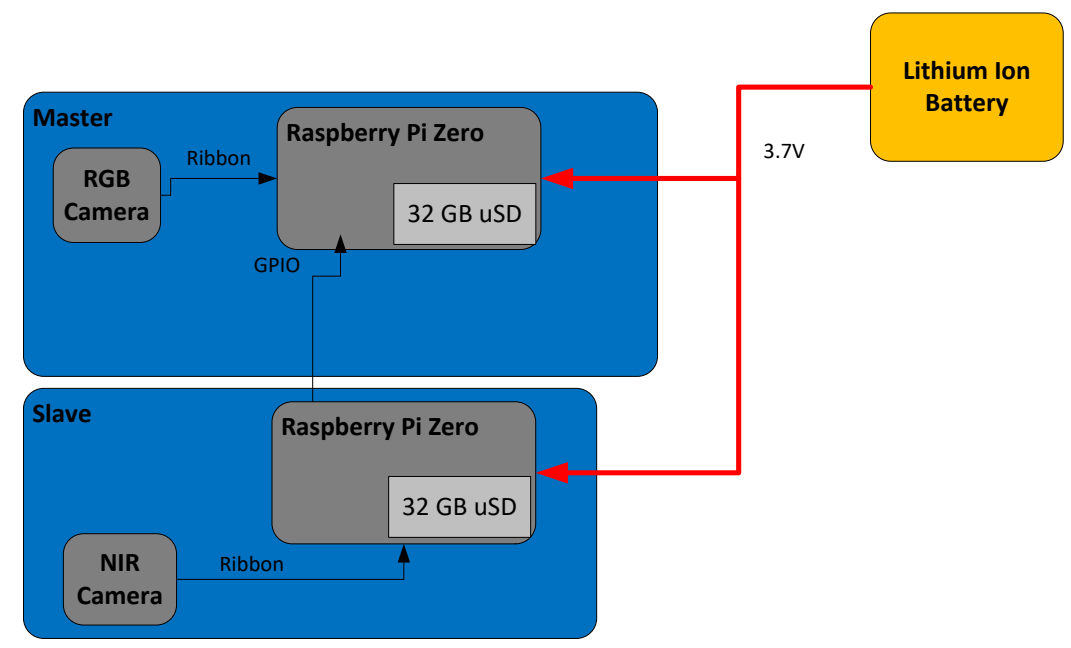

Figure 30: Function Block Diagram of the Experimental NDVI Payload

\subsection{Software Design}

As previously mentioned the NOOBS package was used to install the Raspbian operating system (OS). The hardware boot options were configured for auto login which allow the RPi to boot without typing in a username and password. Booting to the graphics $\mathrm{X}$ terminal was also disabled along with the splash screen in effort to reduce the boot time as much as possible. The flight code for this experimental payload was written mostly in the Python programming language. This was selected over other common languages such as $\mathrm{C}++$ because of the plethora of information and libraries written in Python which are supported by the Raspberry Pi opensource community. The os.sys Python library was also included so that Linux system commands could be used directly with the Python script.

A software flowchart diagram for the master RPi CPU is illustrated in Figure 31. The first blocks of code are dedicated to variable declarations and initializing GPIO pins to inputs or outputs and setting output pins to LOW or "0" value. In case the GPIO pins were being utilize and an unexpected power outage occurred, it is possible that the GPIO pins will be unavailable at to initialize during the next execution of the program, therefore a try statement was employed to attempt to reset the GPIO pins in the event that they are already being used when the program is initialized. Next, the camera is initialized and the parameter values are set. The final portion of the initialization section of code deals with counting the number of folders in the "Images" directory where flight data is stored. An algorithm was implemented to count the number of folders and append the next number to the end of the filename to create a new file folder every time the program is run (e.g. Test1, Test2, Test3, etc.). The next section of code handles the loop structure of the master flight software. An overall loop keeps track of the elapsed time vs. the test time established for the flight. If the elapsed time exceeds the test time then the program ends. While the elapsed time is less than the set test time, another loop checks the value of the read pin and keeps track of the time in between the loops. If the timeout between the loops exceeds the 
specified value of 60 seconds, the program bypasses the main loop and continues to take unsynchronized photos. However, if the timeout is not exceeded then the master CPU waits for the slave CPU to turn the master "read" pin $H I G H$. When the signal on the master "read" pin goes HIGH, the master CPU turns its "write" pin $H I G H$, delays for a moment to stabilize the camera sensor, takes a photo, then turn its "write" pin $L O W$ again then increments a counter.

A similar flowchart diagram for the slave CPU is shown in Figure 32. The initial section of the code with variable initialization, GPIO pin setup, camera initialization, and file checking is identical to the master CPU code with the exception of switching the input/output setup of the GPIO pins. For the slave CPU, the "shutdown" pin is initialized an input pin instead of an output pin. Also, the "read" and "write" pins are reversed for the slave CPU and set to input and output pins, respectively. The main loop for the slave CPU constantly checks whether the master CPU has turned its "shutdown" pin to $H I G H$, or a value of "l". If so, the program ends. Otherwise, the slave CPU checks its "read" pin and if the pin goes $H I G H$, then it takes a picture and waits for the "read" pin to turn $L O W$ again. When its "read" pin turns $L O W$, a counter is incremented and the loop continues. If the time between loops is less than the timeout value of 60 seconds, the program bypasses the main loop and continues to capture unsynchronized photos until the "shutdown" pin turns $H I G H$. For reference, both master and slave flight code is provided in Appendix C. 


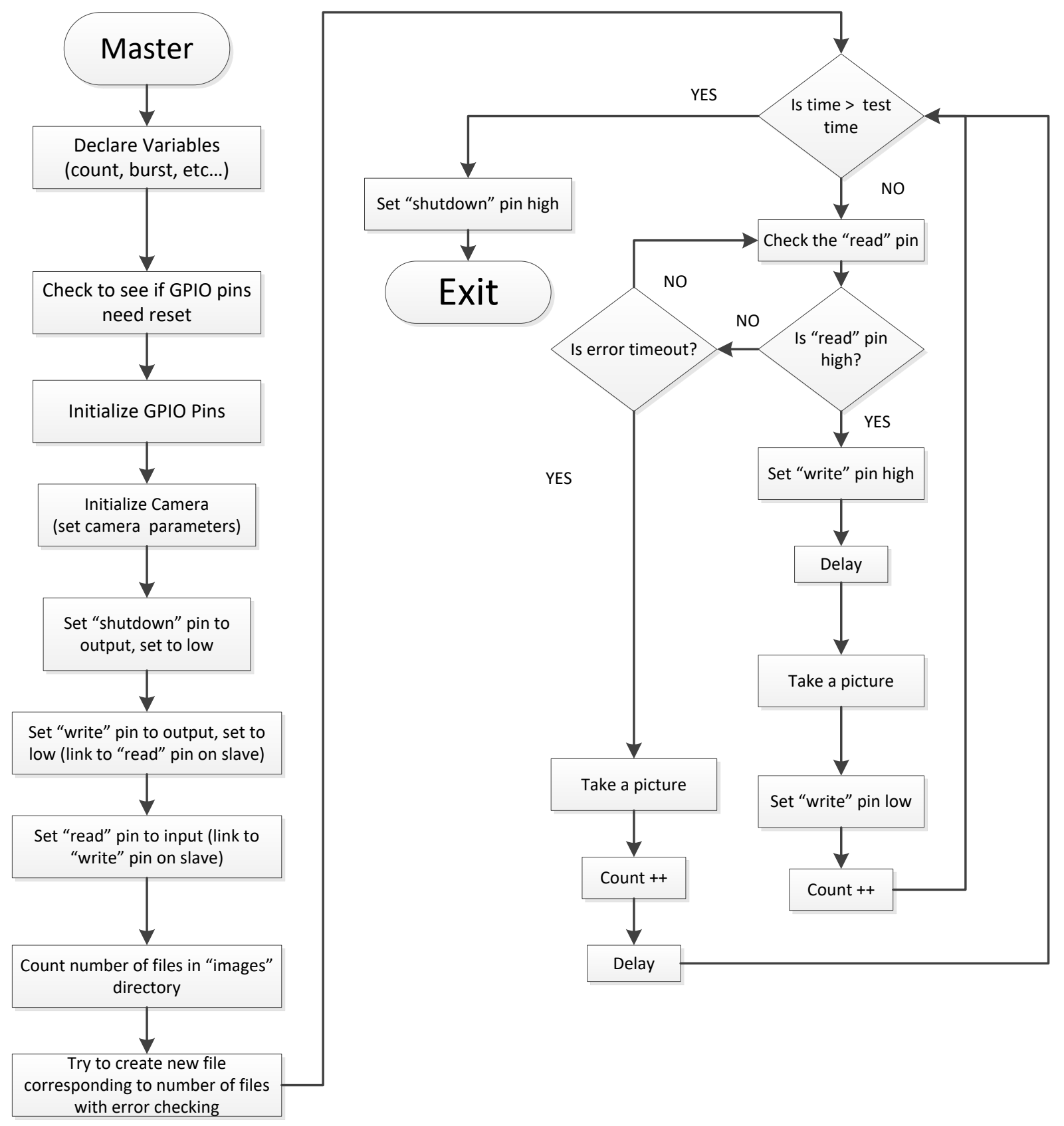

Figure 31: Master CPU Software Flowchart 


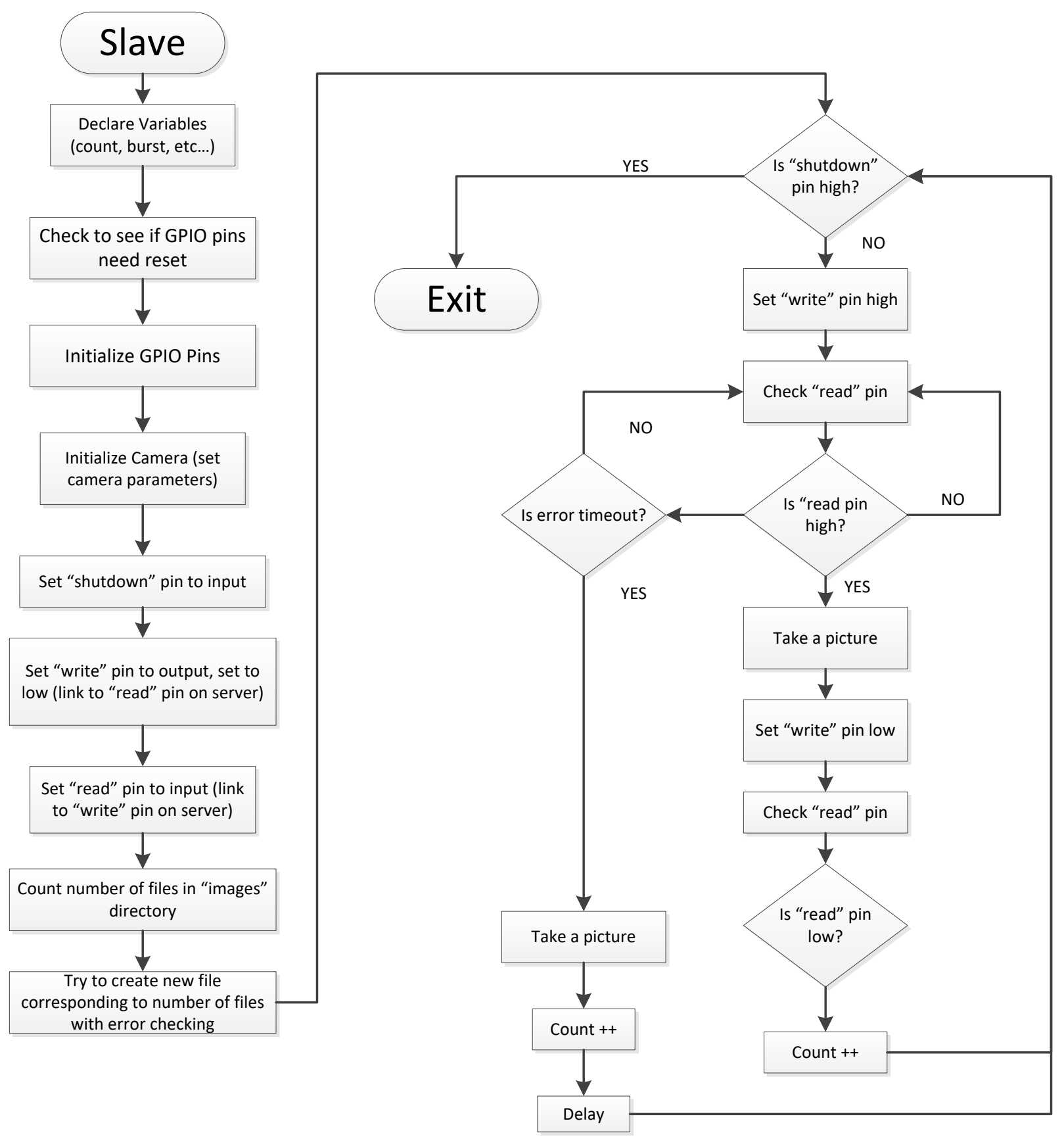

Figure 32: Slave CPU Software Flowchart

\section{$3.4 \quad$ Prototype Setup}

Initial testing was done on a portable prototype testing platform. The platform consisted of a slight variation of the final design for the NDVI Cubesat/UAV payload; however, the main functionality remained the same. RPi 2 CPU boards were used instead of RPi Zero CPUs because they have 4 USB ports which did not require the use of an on-the-go (OTG) micro USB 
to USB adapter or a USB Hub extender for keyboard connections and wifi adapters as with the RPi Zero. Also, a larger 7.4 V LiPo battery was used for extended life which required a separate power distribution board to regulate the power.

A photo taken during initial testing of the portable NDVI prototype platform can be seen in Figure 33. The photo shows the view field of the RGB camera on the TV screen which are both connected to the master RPi. A hand is waving in the view field of the RGB camera to demonstrate functionality of the camera.

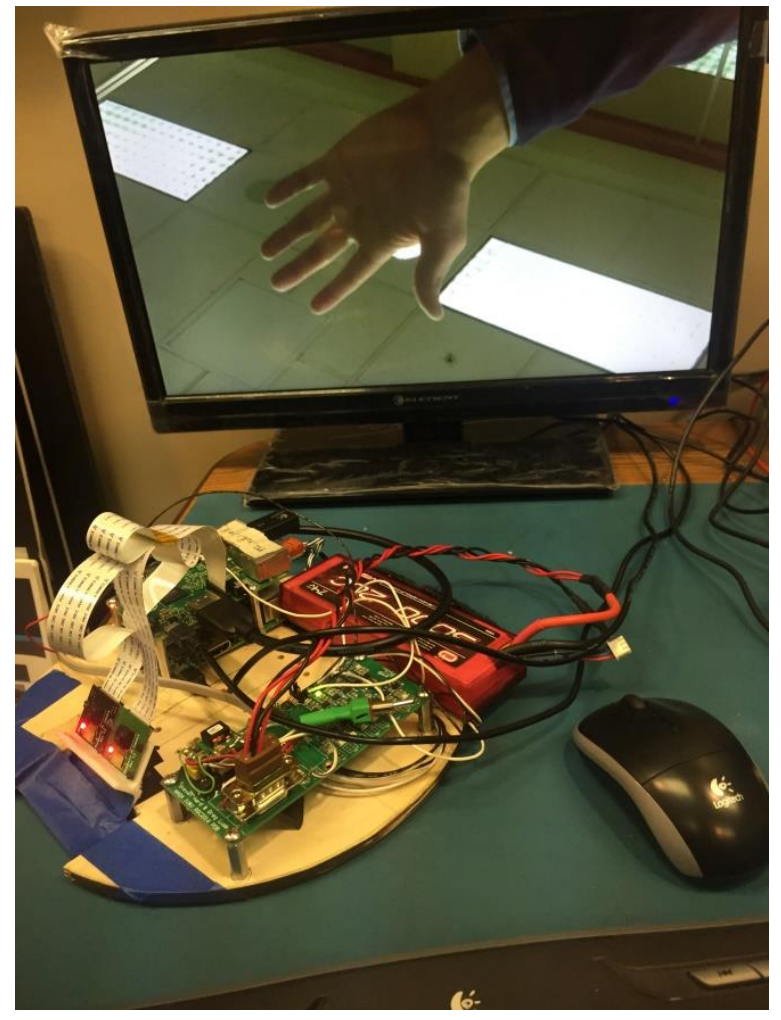

Figure 33: Portable NDVI Prototype Platform Taking an Image

\subsection{PCB Design}

A custom PCB was designed to provide voltage regulation circuitry as well as to provide a common interface between the two RPi boards and the Adafuit 10 DOF IMU. The following section each explains the PCB design process and includes electrical schematics with the PCB design layout for the top and bottom layers. Although the 10DOF IMU is included in the PCB design as a payload option, it was not used in the analysis of this research. However, the IMU is still discussed in this section as part of the PCB design process. 


\subsubsection{Electrical Schematic}

The printed circuit board design process started by creating a schematic using the Altium Designer PCB design software environment. First, the power circuit was designed using the datasheet for the voltage converter as a reference [44]. This voltage regulation circuit is shown in Figure 34. It can be seen from the schematic that the battery voltage (BAT) gets regulated to $5 \mathrm{~V}$ through the LM2731. The next step was to create footprint libraries for the RPi and the IMU devices. Figure 35 shows all the various networks (nets) assigned to the pins on the RPi footprint whereas Figure 36 shows the nets assigned to the pins of the 10 DOF IMU header footprint.

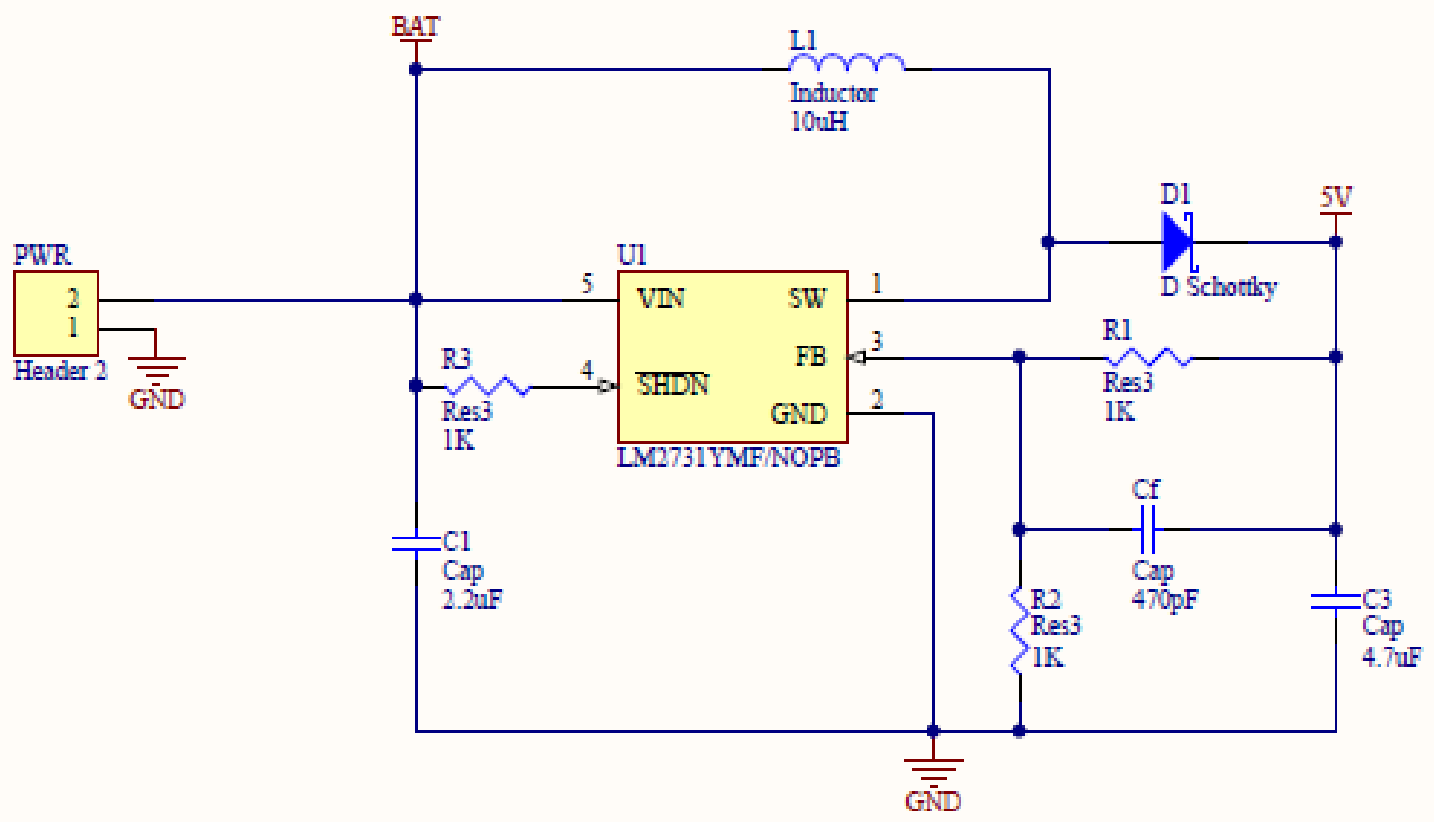

Figure 34: Voltage Regulation Circuit Schematic 


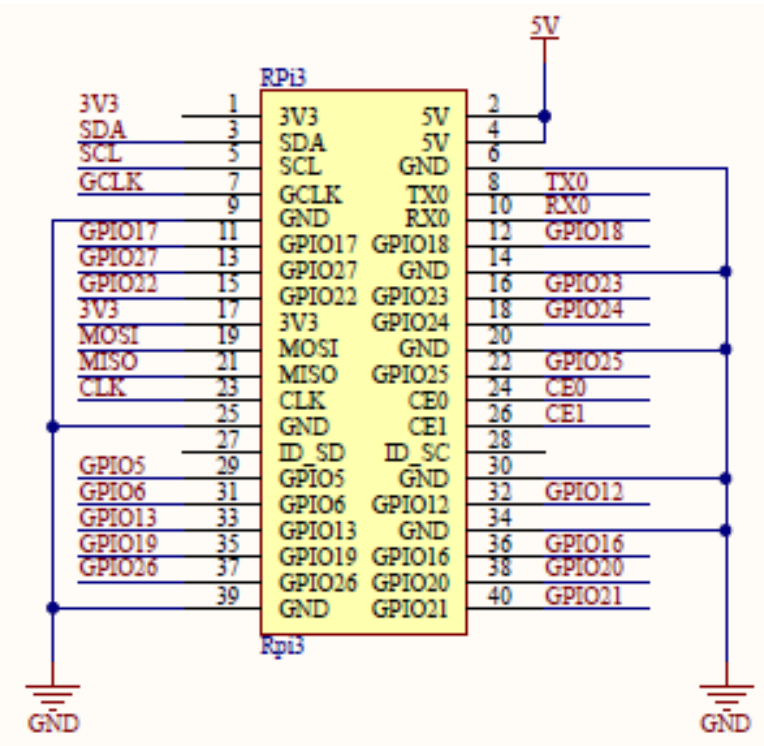

Figure 35: Raspberry Pi Header Pinout Schematic

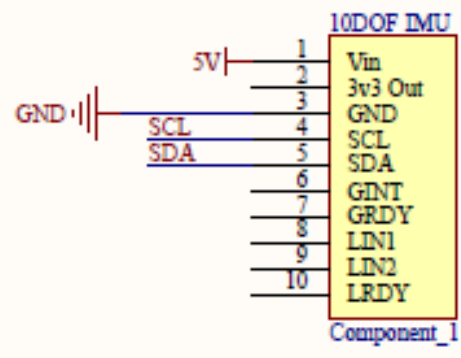

Figure 36: Adafruit 10DOF IMU Header Pinout Schematic

\subsubsection{PCB Layout}

Once the schematic footprint libraries were created and a basic schematic file was built, the actual PCB design could be laid out. This started with the physical design requirements being traced as an outline layer of the PCB. Then slots were drawn in the upper edge of the board outline to create cutouts just wide enough for the two camera lens to fit within the gap. Next, holes were placed in the positions according to the physical design specifications. With the physical design requirements met, the remaining components could then be placed anywhere within the perimeter of the board outline. EAGLE files were imported for the RPi 2, RPi camera modules, and 10 DOF IMU board to create an outline of each components. Once the imported boards were set in place, all layers of the components were deleted except the board outline and drill holes. Since the original PCB design included RPi 2 boards, the placement of the RPi footprint was rather limited in that it could only be flipped by $180^{\circ}$. Therefore, the RPi 2 CPU board was centered somewhat between the left and right while offset somewhat to the bottom to 
allow space for the two camera modules at the top. An additional 40 pin header was broken out to provide a connection for the stack of two RPi 2 boards. The two camera modules were placed as close to each other as physical possible and centered along the top of the PCB outline. Lastly, the IMU was positioned such that it could be mounted underneath the RPi 2 stack. Images of the PCB design are provided in Figure 37 where the top layer can be seen on the left and the bottom layer which is mostly used for a solid ground plane can be seen on the right. The top layer has the main power tracing and voltage regulation circuit whereas the bottom has traces for the $\mathrm{I} 2 \mathrm{C}$ connection between the IMU and the RPi.
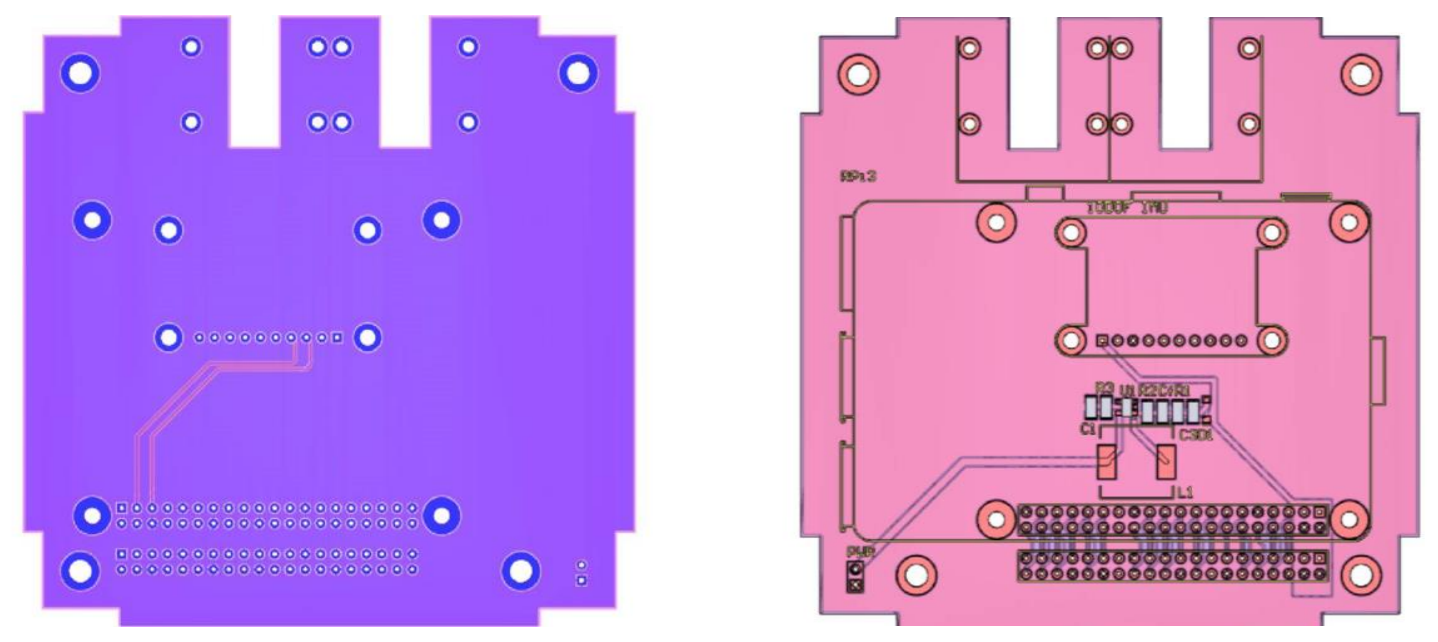

Figure 37: PCB Design of the NDVI CubeSat/UAV Payload

Top Layer (left) and Bottom Layer (right)

\subsection{PCB Assembly}

This section discusses the level of soldering involved in the payload PCB design and how to best take advantage of different skillsets to populate the PCB with the various electrical components incorporated in the design. The procedure for mounting the payload components to the PCB and associated wiring connections are also described.

\subsubsection{Soldering}

One advantage that this payload design has for academia is that it offers a simple design but yet is challenging enough promote aspects of learning on various levels. For instance, soldering the 40 pin through-hole header pin connector is quite trivial yet tedious. This task would be great to assign to someone just learning how to solder to develop their soldering skills. The voltage regulation circuit, however, is quite compact and incorporates tiny surface mount components. At the same time, it is not overly complex nor is it spread all across the board. This 
is another great way for someone with more advanced soldering skills to hone their capability of working with surface mount components. At any rate, the design is kept simple and should not require an extensive amount of time to populate. A photo taken after PCB assembly is shown in Figure 38. A female version of the 40 pin socket connector was also soldered in place to accommodate the RPi stack. A list of electrical components used in the PCB design is included in Appendix D.

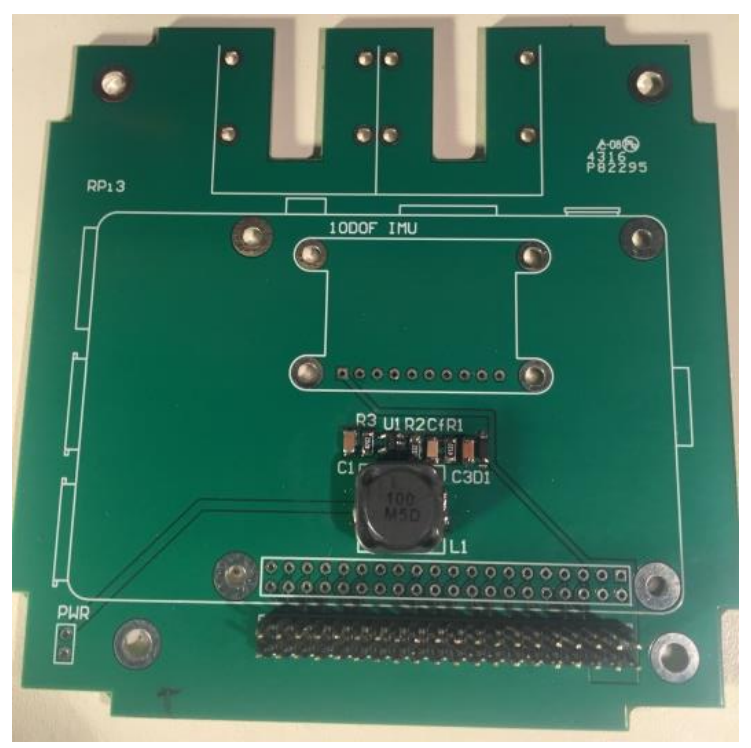

Figure 38: Assembled PCB after Soldering

\subsubsection{Mounting and Wiring}

With the electrical components fully populated on the PCB, the main components were ready to be mounted and necessary wiring to be routed. A design decision was made to incorporate RPi Zero modules instead of RPi 2 modules to reduce overall weight which is crucial for UAV and cubesat applications. Since two of the four mounting holes for the RPi Zero and RPi 2 overlap, a PCB redesign was not required. A cantilevered mounting technique was used for each of the RPi Zero boards which also reduced the overall height of the experimental payload. Before the slave RPi Zero was mounted, the NIR and RGB cameras were fastened in place using M2x4 mm stainless steel screws and plastic spacers, and the ribbon cables were then attached to the camera modules. The master RPi Zero was mounted using M2.5x4 mm screws and $6 \mathrm{~mm}$ aluminum hex female-female standoffs. The slave RPi Zero was mounted using the same fasteners but with $10 \mathrm{~mm}$ aluminum hex female-female standoffs to create an offset in board height between the master and slave RPi boards. Once the master and slave RPi boards were mounted, the camera ribbon cables were attached.

The following photographs show different angles of the fully mounted and wired payload assembly. In Figure 39, the payload can be seen from a top view. In this view, the connections between the master and slave RPi boards can be seen. Pins 2 and 4 provide redundant power to 
the slave while pins 6 and 14 provide redundant grounds. For the master RPi, pin 12 is used as the "shutdown" pin and pins 16 and 18 are used as "write" and "read" pins, respectively. A toggle switch was connected to pin 22 to take the place of a timed loop for field testing. For the slave RPi, pin 12 was also used as the "shutdown" pin while pins 16 and 18 were the "read" and "write" pins, respectively. In Figure 40, a side view shows the camera modules mounted with plastic spacers underneath the cantilevered slave RPi. The ribbon cable for the NIR camera ribbon cable is routed underneath the slave RPi board as well. A bottom view of the camera lenses and the battery is pictured in Figure 41. Note the filter used on the NIR camera lens. A square piece of exposed developed film was used as the filter to block out almost all visible light from the NIR camera lens. Therefore, it is only seeing light in the IR spectrum.

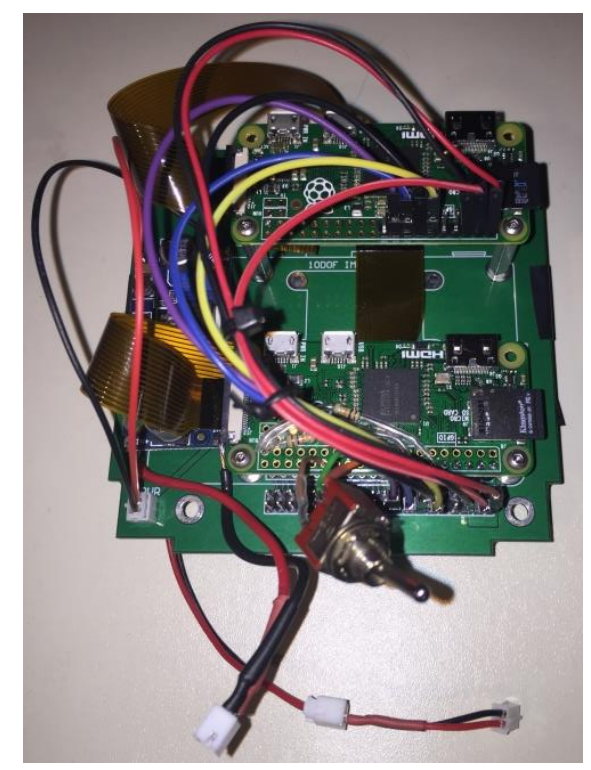

Figure 39: Top View Photo of Payload Assembly

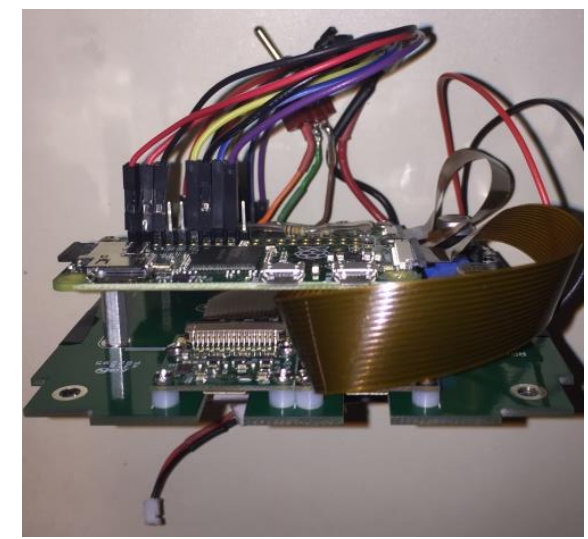

Figure 40: Side View Photo of Payload Assembly 


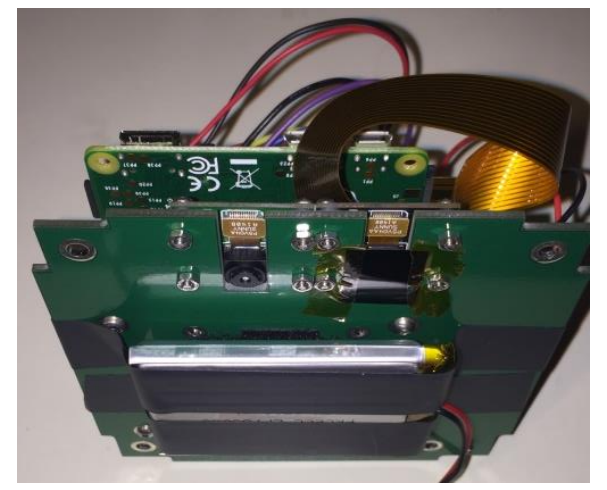

Figure 41: Bottom View Photo of Payload Assembly 


\section{Chapter 4 | Results}

Post-processing procedures used for data analysis are described in this section along with functional testing aspects that should be considered prior to flight tests or launches. Results from a UAV flight at $10 \mathrm{ft}$ above ground level (AGL) and a general aviation flight are shown at $250 \mathrm{ft}$, $500 \mathrm{ft}, 1000 \mathrm{ft}, 2000 \mathrm{ft}$, and $3000 \mathrm{ft}$ AGL as proof of concept.

\subsection{NDVI Post-Processing}

\subsubsection{GIMP}

The GNU Image Manipulation Program (GIMP) is an open-source graphics editing package similar to Microsoft Photoshop. This software package was downloaded for free from the GIMP website and used to create NDVI images using raw RGB and NIR photos [47]. There are many tutorials available online which demonstrate the tools and capabilities of GIMP for editing graphics and photos. The main functions necessary to perform this research analysis are overlaying images for alignment and the use of the "add", "subtract", and "divide" modes in the GIMP layers toolbar.

\subsubsection{Image Layering}

The GIMP software is based on the concept of image layering to perform certain operations of layers overlaid on top of one another. Each layer that is imported into GIMP can be turned on or off for viewing. The layer that is on placed in the top of the image layer stack in the "Layer Toolbox" panel is the layer that is shown on top. If the transparency of the top layer image is reduced, the image that is next in line to the top and turned on for viewing will become visible. If the operation mode is set to anything other than "normal", then the selected operation will be performed with the next image in line to the top on the top image. For example, in order dependent operations such as subtraction, to subtract $Y$ from $X, Y$ should be placed on top of $X$ in the "Layer Toolbox" panel.

The images shown in Figure 43 were taken during field testing at Dorsey's Knob State Park in Morgantown, WV. The image on the left is the VIS image and the image on the right is the NIR image. Both of these images were imported as layers into GIMP. Each image was desaturated by navigating under the "Colors" tab and selecting "Desaturate", then selecting the shade of grey based on "Lightness". This was done because the color data is not used in the NDVI analysis, but rather only the brightness data. Once each photo was desaturated to black and white, an auto exposure was applied to both images by once again navigating under the "Colors" tab and selecting "Levels", then selecting "Auto" for adjusting color level. With each image desaturated and auto-exposure applied, duplicates of each image were then created. In 
order to keep track of each image layer, they were renamed as, "NIR_top", "VIS_top", "NIR", and "VIS" corresponding to the NIR and VIS images from top to bottom. If the images need to be aligned, it is helpful to add a new layer at the bottom of the image layer stack to serve as a blank background.

Equation (1) was implemented using GIMP to stack the images and perform operations on each pixel of selected images. The denominator of Equation (1) was first executed by turning off the "NIR_top" and "VIS_top" layers and then selecting the "Addition" mode. Then, these two images were merged into one by navigating under the "Layers" tab and selecting "Merge Down". The resulting image layer was renamed "NIR+VIS" and then turned off from view. Next, the "VIS_top" layer was placed at the top of the layer stack directly above the "NIR_top" layer and both image layers were turned on for view. The "Subtract" mode was then selected and once again the two images were merged into one with the "Merge Down" function. The resulting image was renamed "NIR-VIS". This reordering process is necessary due to the order dependent nature of the subtraction operation, so the "VIS_top" layer serves as the subtrahend and the "NIR_top" is the minuend. Thus, the merged "NIR-VIS" image is the resulting difference. Similar to the previous step, the "NIR+VIS" layer was placed above the "NIR-VIS" image but this time the "Divide" mode was selected. And once again, two intermediate images were merged together with the "Merge Down" function and the resulting image renamed "NDVI". A color map of the resulting NDVI image was created using magenta (ff00ff) as the lowest NDVI value and red (ff0000) as the highest value. Figure 42 illustrates the steps described to perform the NDVI post-processing on aligned NIR and VIS images that have been desaturated and autoexposed. In the top left, the four image layers can be seen with the "NIR" layer selected and the "Addition" mode applied. In the top right, the resulting "NIR+VIS" layer can be seen along with the "VIS_top" top layer selected and the "Subtract" mode applied. In the bottom left, the "NIRVIS" layer can be seen along with "Divide" mode applied. Finally, the bottom right shows the final "NDVI" layer resulting from merging the two intermediate image layers which represent the numerator and denominator of Equation (1). The raw NDVI image and NDVI gradient color map are shown Figure 44. 


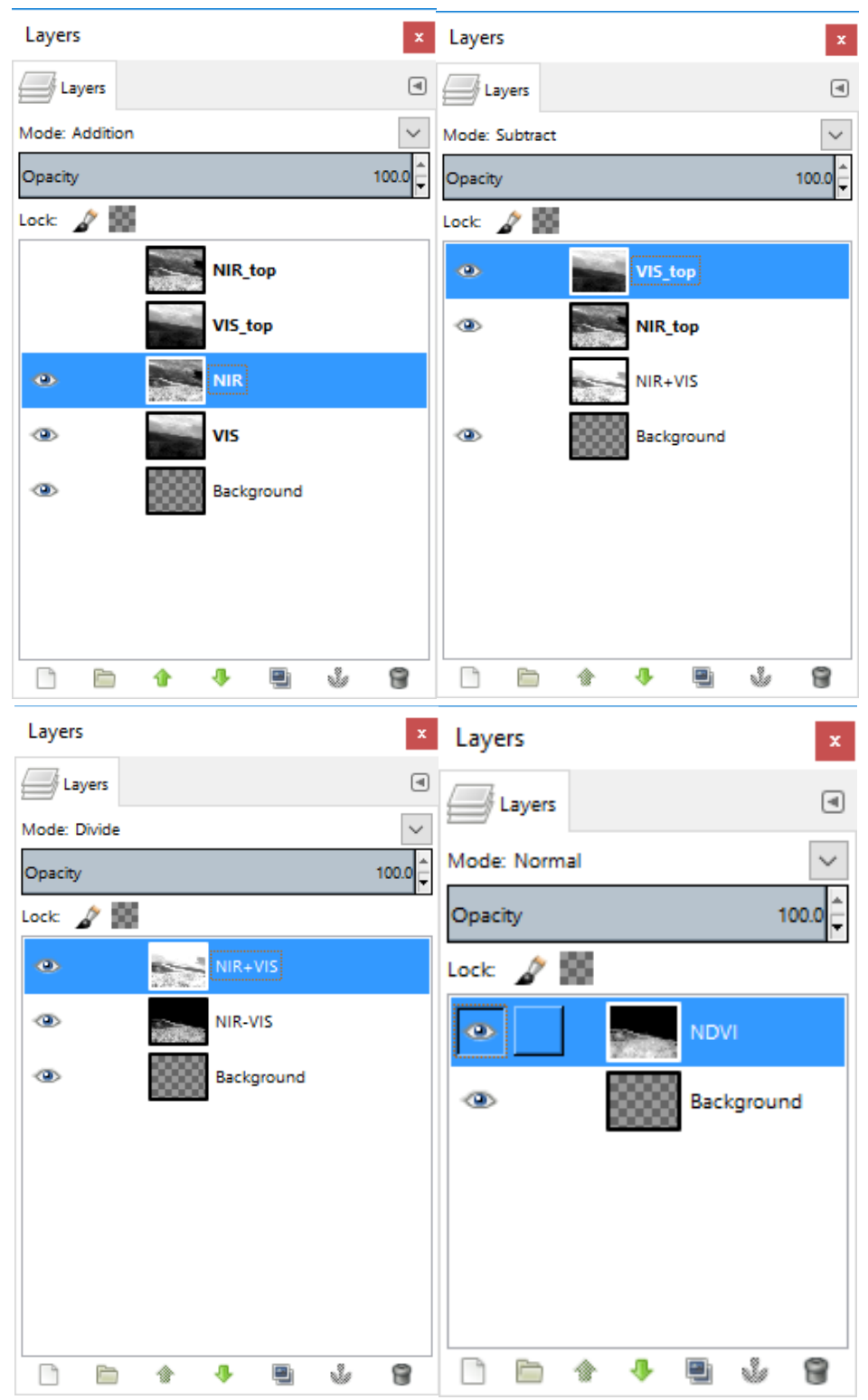

Figure 42: GIMP Image Layer Panels Illustrating NDVI Post-Processing Procedure (top leftAddition, top right-Subtract, bottom left-Divide, bottom right-Merge) 


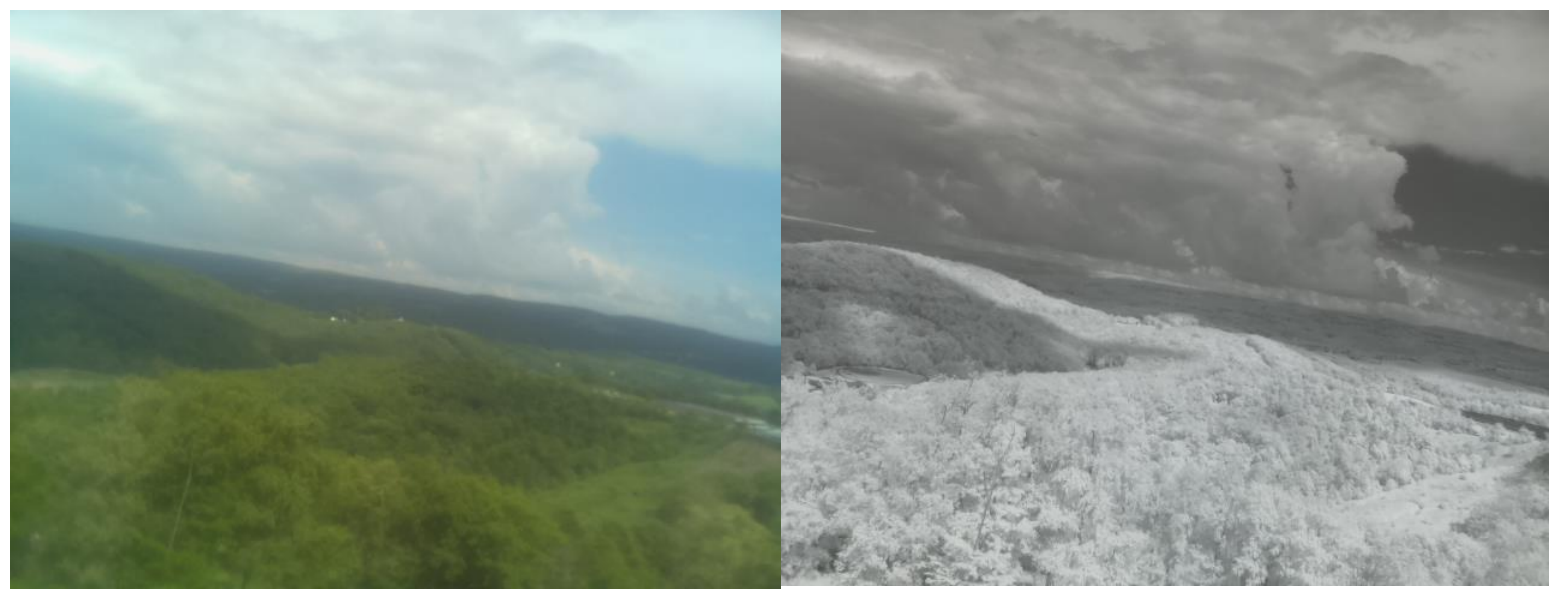

Figure 43: Original RGB (left) and NIR (right) Images Captured during Field Testing

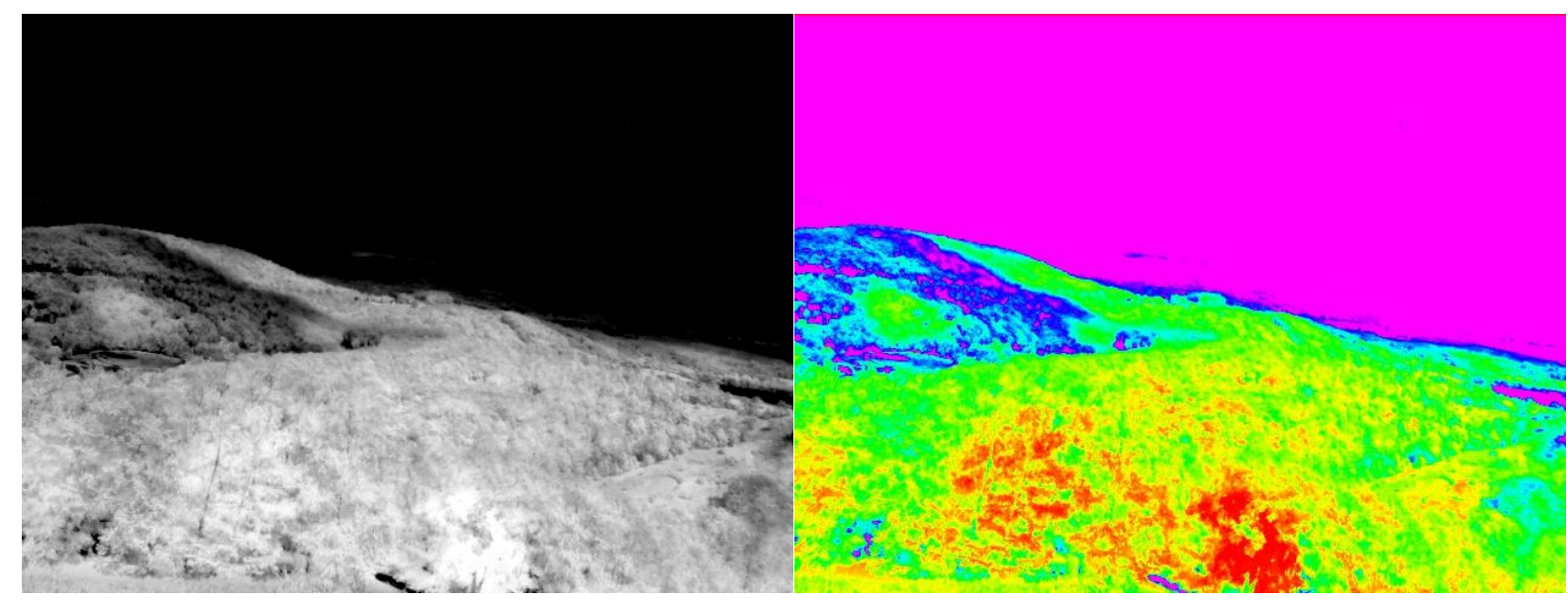

Figure 44: Raw NDVI and NDVI Gradient Color Map Results from Field Testing

\subsubsection{Image alignment}

Even though particular care has been taken to ensure the two cameras used in the experimental payload take images simultaneously, there is still a slight delay between the two cameras on startup that cannot be avoided. For images that are taken from afar, the effects of the slight delay between the cameras are minimal. However, as the frame of the image gets closer to the camera lenses, the effect becomes more pronounced. Moreover, there is a slight offset in the lenses of the two cameras which becomes more noticeable as the image frame becomes closer to the camera lenses, even if the cameras are stationary. An example of the image offset can be seen in the close-up images shown in Figure 45. 


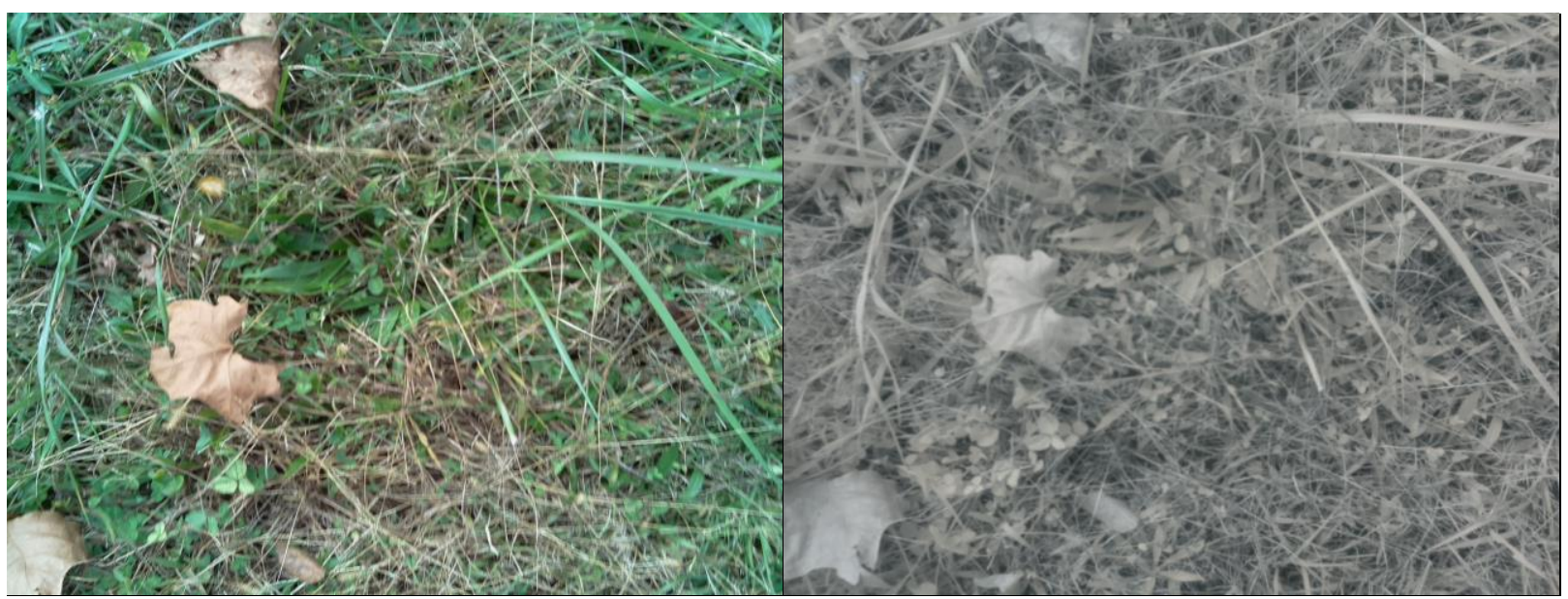

Figure 45: Close-up RGB (left) and NIR (right) Images Showing Slight Offset

It is essential that the RGB and NIR images are properly aligned in order to maximize the integrity of the data obtained through the NDVI post-processing procedure. This was done on the images shown in Figure 45 by importing them into GIMP as layers. The NIR image was placed on top of the RGB image and the transparency was set within a range of 50-70\% such that the RGB image clearly showed through the NIR image. This is illustrated in Figure 46. The "Move Tool" was then used to translate the NIR image over top of the NIR image such that the pixels of the image are in alignment with one another. If necessary, the "Rotate Tool" can also be used to rotate the image if there appears to be any rotation that occurred between the images captured. In effort to ensure the most optimal alignment, it is helpful to zoom in on a reference point (in this case a leaf or blade of grass) and move the top image slightly by a single pixel in each direction when the image seems aligned. If the image fuzziness improves, repeat this process. This will help to ensure that the error of alignment is to within at least $2 \Delta p$, i.e. the two images are aligned to within two pixels in either direction. Ideally the images are completely aligned with $0 \Delta p$ to maximize data integrity. For this research however, it was deemed that a small amount of error was acceptable for initial results analysis and proof of concept. Figure 47 shows the two images after the NIR image has been aligned with the RGB image. Once aligned, each image was then auto-cropped by navigating under the "Image" tab and selecting "Autocrop Image". The aligned and cropped RGB and NIR images can be seen side by side in Figure 48. Using the procedure described in section 4.1.2, the raw NDVI and NDVI color map images were created and are shown in Figure 49. 


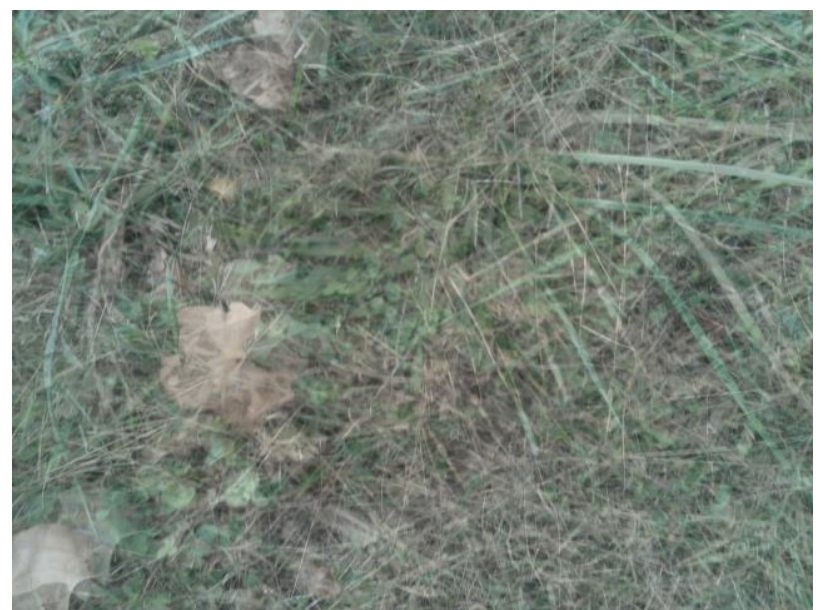

Figure 46: NIR Image with 50\% Transparency Showing RGB Image Offset

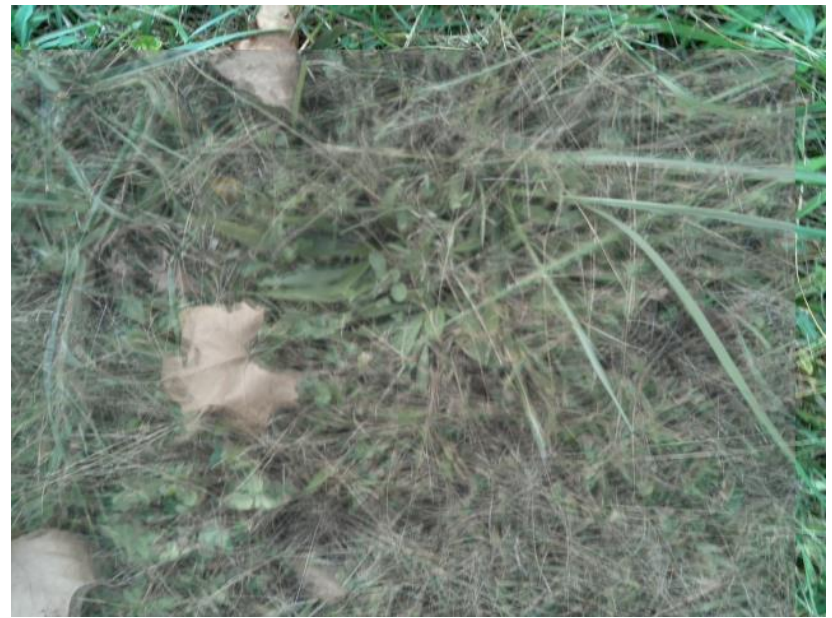

Figure 47: NIR Image with 50\% Transparency Showing RGB Image Alignment

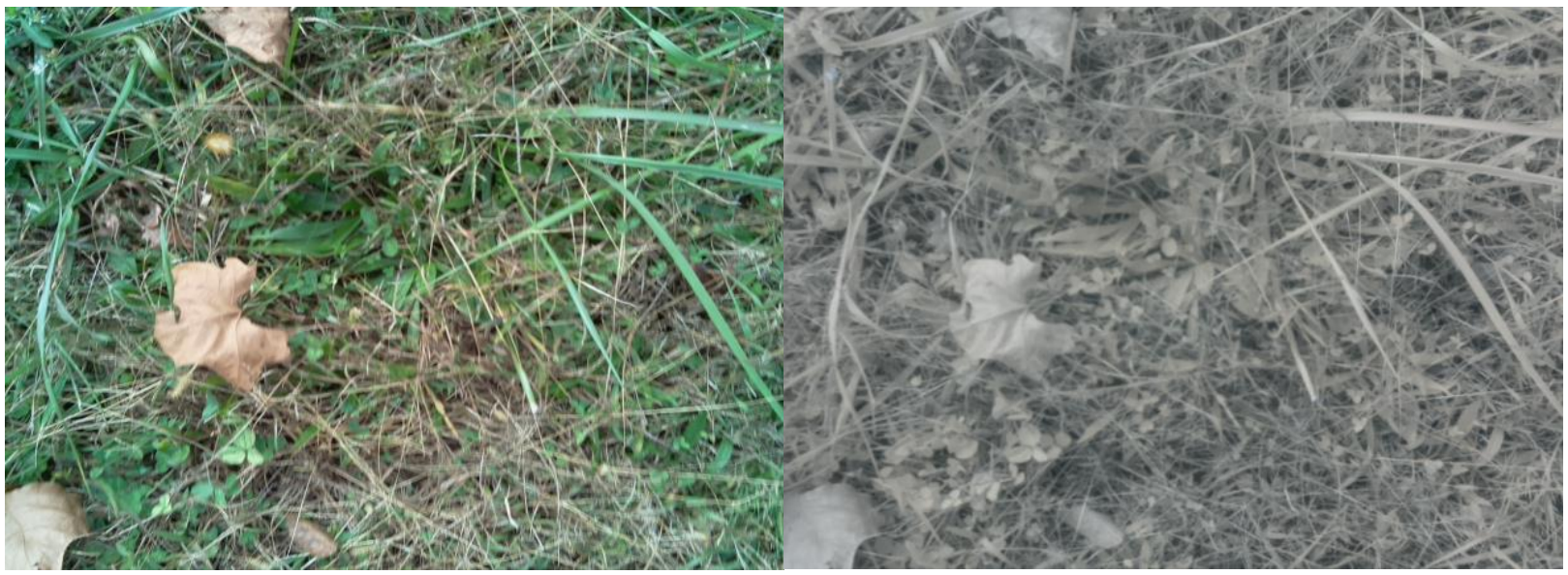

Figure 48: Cropped RGB (left) and NIR (right) Images of Close-up Still Shot 


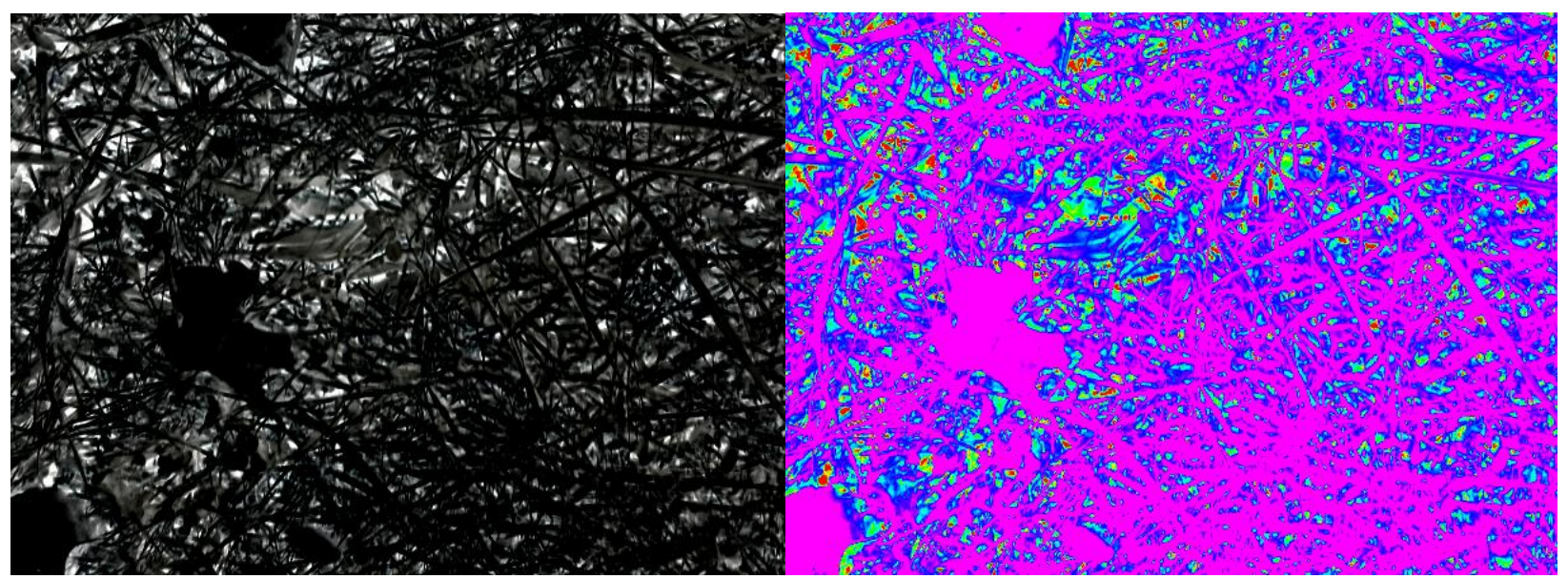

Figure 49: Raw NDVI (left) and NDVI Color Map (right) of Close-up Still Shot

As the image frame gets further away, alignment is not as much of an issue for still shots where the cameras are stationary. A mid-range landscape seen was captured in Figure 50. At first glance, it appears that the RGB and NIR images are in perfect alignment. However, when the images were overlaid and transparency reduced for the top image, a very slight offset was still observed. Once the images were aligned, the NDVI post-processing method outlined in section 4.1.2 was applied once again. The resulting raw NDVI and NDVI color map images are illustrated in Figure 51.

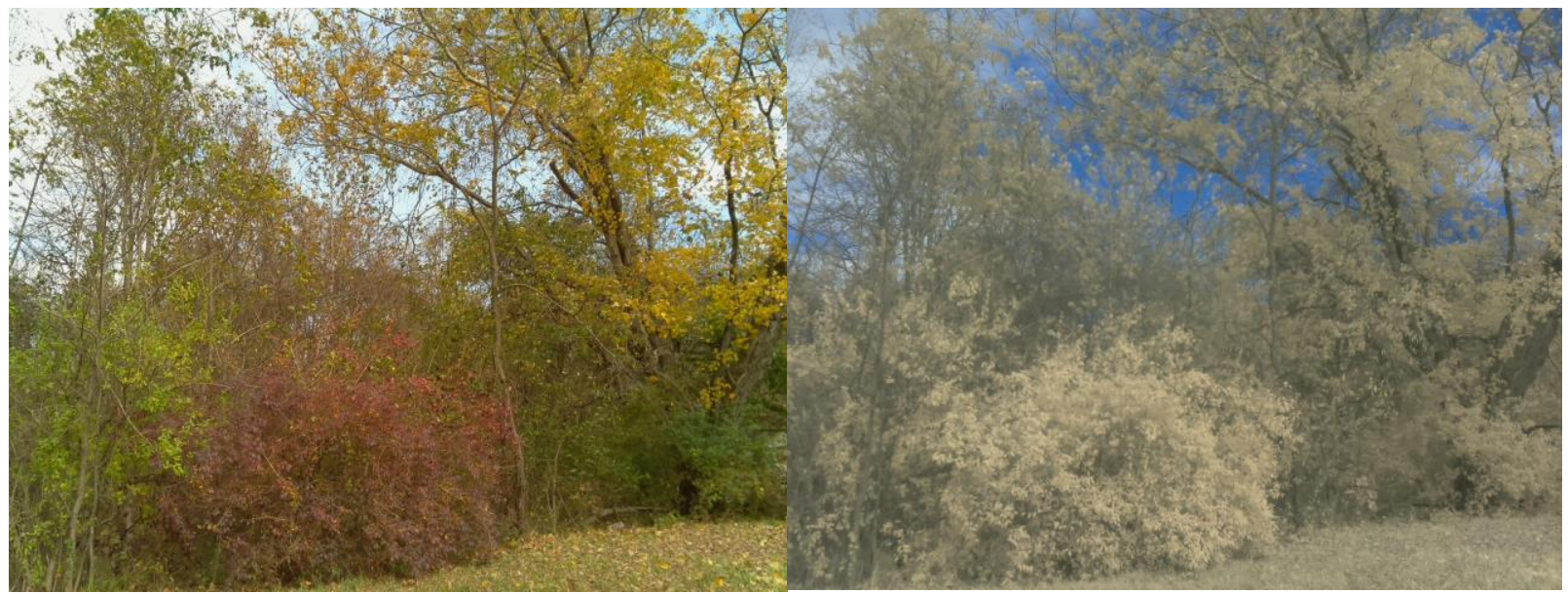

Figure 50: Original RGB (left) and NIR (right) Images of Landscape Still Shot 


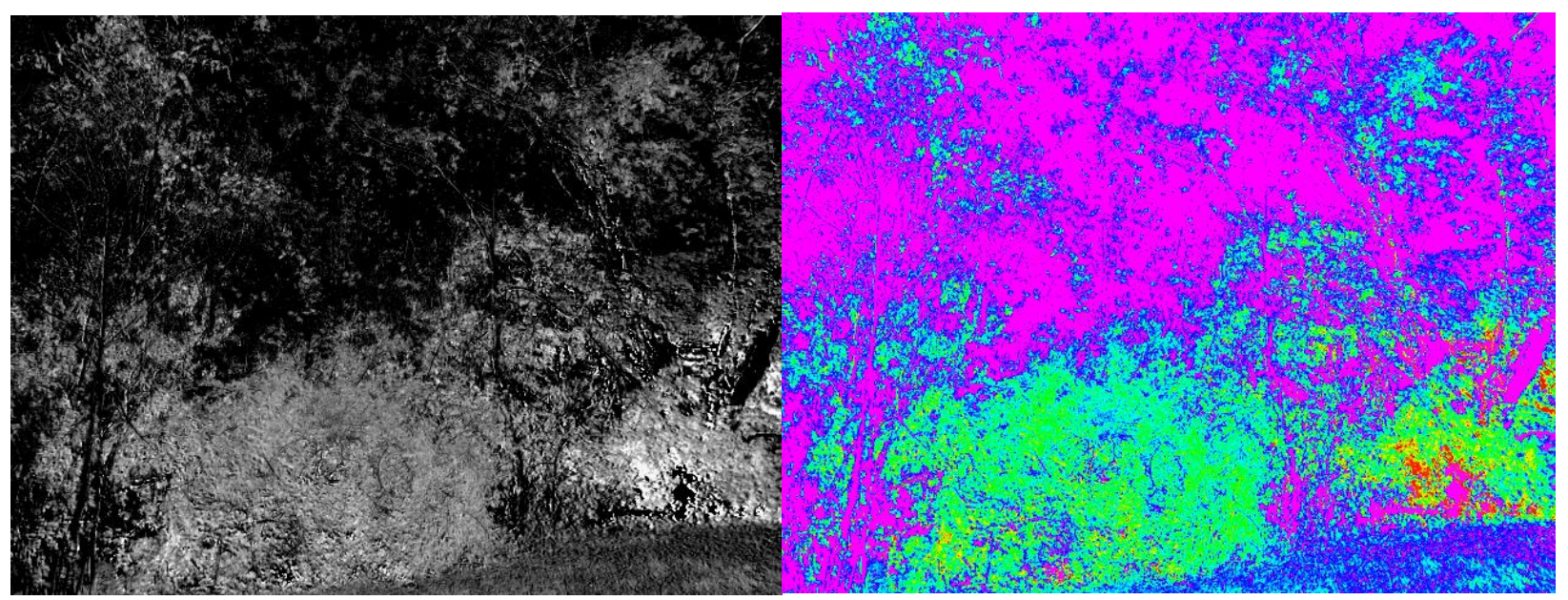

Figure 51: Raw NDVI (left) and NDVI Color Map (right) of Landscape Still Shot

\subsection{Functional Testing}

\subsubsection{Timing}

As previously mentioned, there is a slight inherent delay between the images taken by each camera of the NDVI payload due to the loop checking for digital GPIO pins turning high and low. This delay is characterized by the test image shown in Figure 52. The images taken by the two cameras on the first iteration of the loop have a slight offset between them of about 0.5 seconds. Then, after successive loops the next images captured are synchronized but add an additional delay of about 0.5 seconds. Therefore, the fastest framerate that can be obtained is 2 fps. Figure 52 demonstrates the aforementioned timing offsets. The top left shows the RGB camera timing on the first iteration while the top right shows the NIR camera on its first iteration. The slave CPU captures a NIR camera image and then about 0.5 seconds later the master CPU captures the RGB image. After another 0.5 seconds, the slave CPU captures another NIR image shown in the bottom right while the master CPU captures an RGB image simultaneously with almost no offset as shown in the bottom left. An additional time delay of 0.5 seconds was implemented prior to the master CPU image capture such that the overall framerate was adjusted to be close to $1 \mathrm{fps}$ for flight testing to reduce the overall amount of data to analyze. 


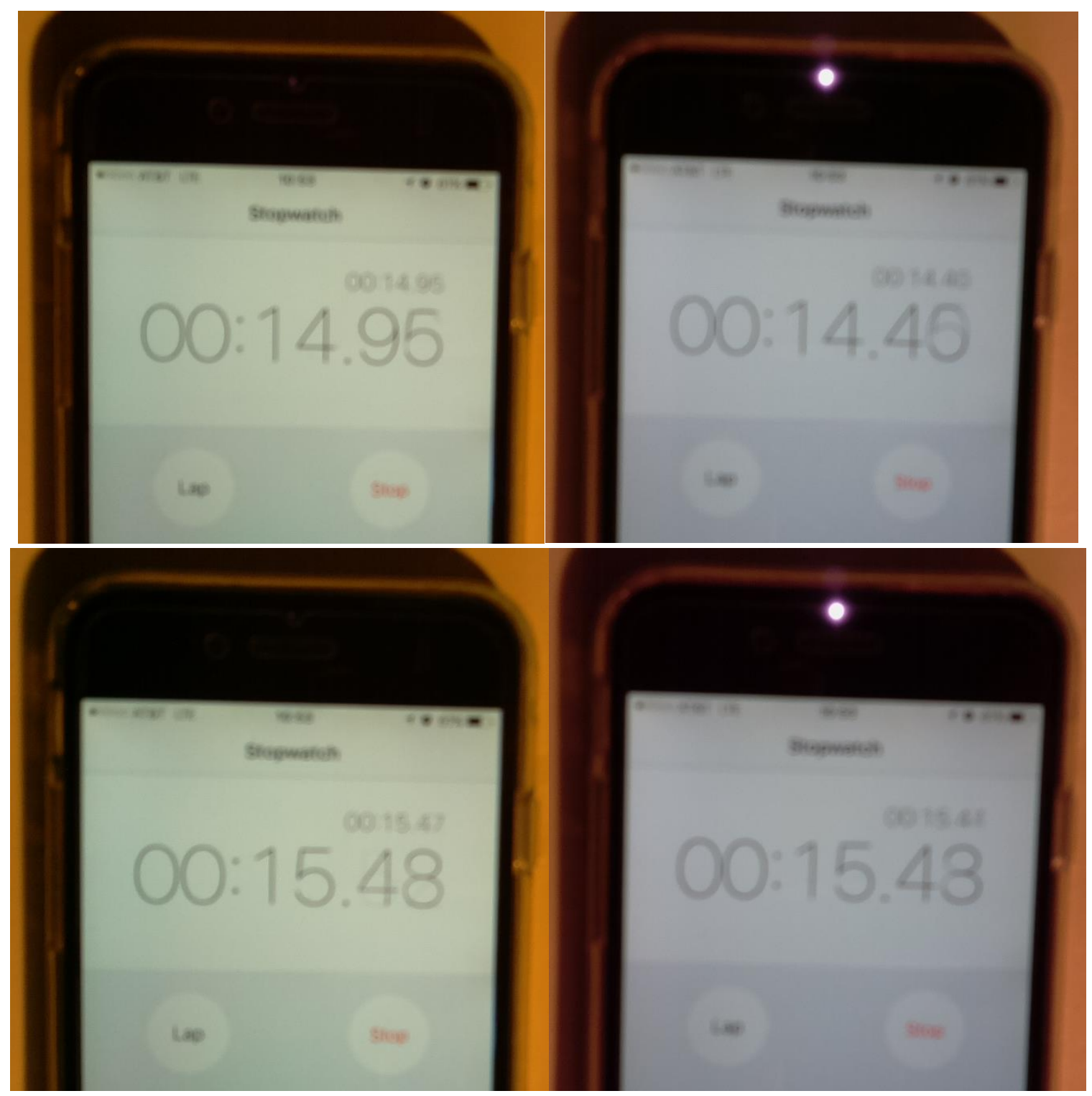

Figure 52: Timing Sequence of the RGB Camera (left column) and NIR Camera (right column) Image Capture on the First Iteration (top row) and the Second Iteration (bottom row)

\subsubsection{Light Filtering}

The NIR camera is essentially the same camera module used in the RGB camera; however the IR filter which is commonly installed in most CMOS type digital camera lenses has been removed. This is the reason is it called the Pi No-IR camera. However, for NDVI analysis, the NIR camera must capture light above the visible spectrum only; therefore an external filter must be applied to the NIR camera lens. There were two different visible light filters tested for performance analysis during functional testing, a blue filter and a black filter. The blue filter was provided by the manufacturer with camera module. The black filter was cut from piece of 
exposed developed photographic film. A still shot of a wooded forest line along with the NIR camera with blue filter is shown in Figure 53. The resulting raw NDVI and NDVI color map are presented in Figure 54. The same image frame was attempted to be captured with the black filter installed on the NIR camera in Figure 55 with the resulting raw and color mapped NDVI illustrated in Figure 56. Upon first glance there was not an obvious difference observed between the two VIS filters, however a closer inspection revealed that the black filter resulted in a wider distribution of NDVI values showing more prominent areas of healthy vegetation. Therefore, the black VIS filter was applied for the remaining testing.

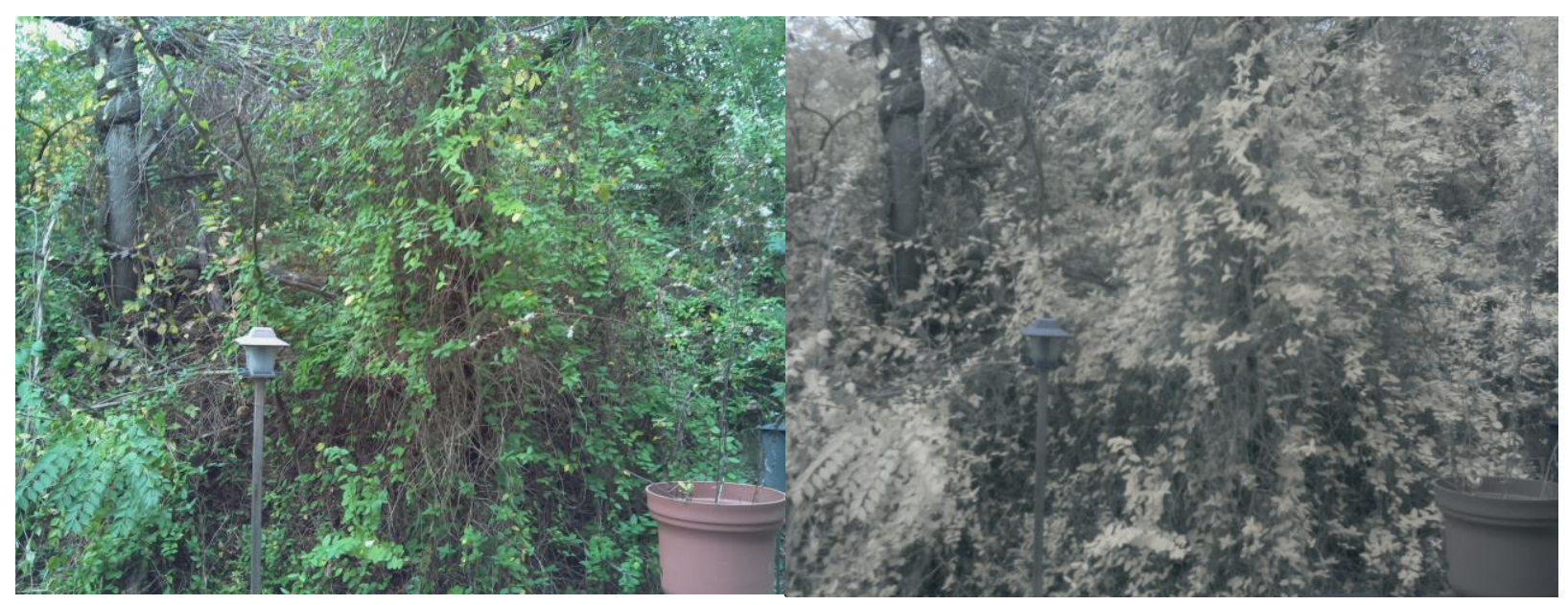

Figure 53: Original RGB (left) and NIR (right) Images of Landscape Still Shot with Blue Filter

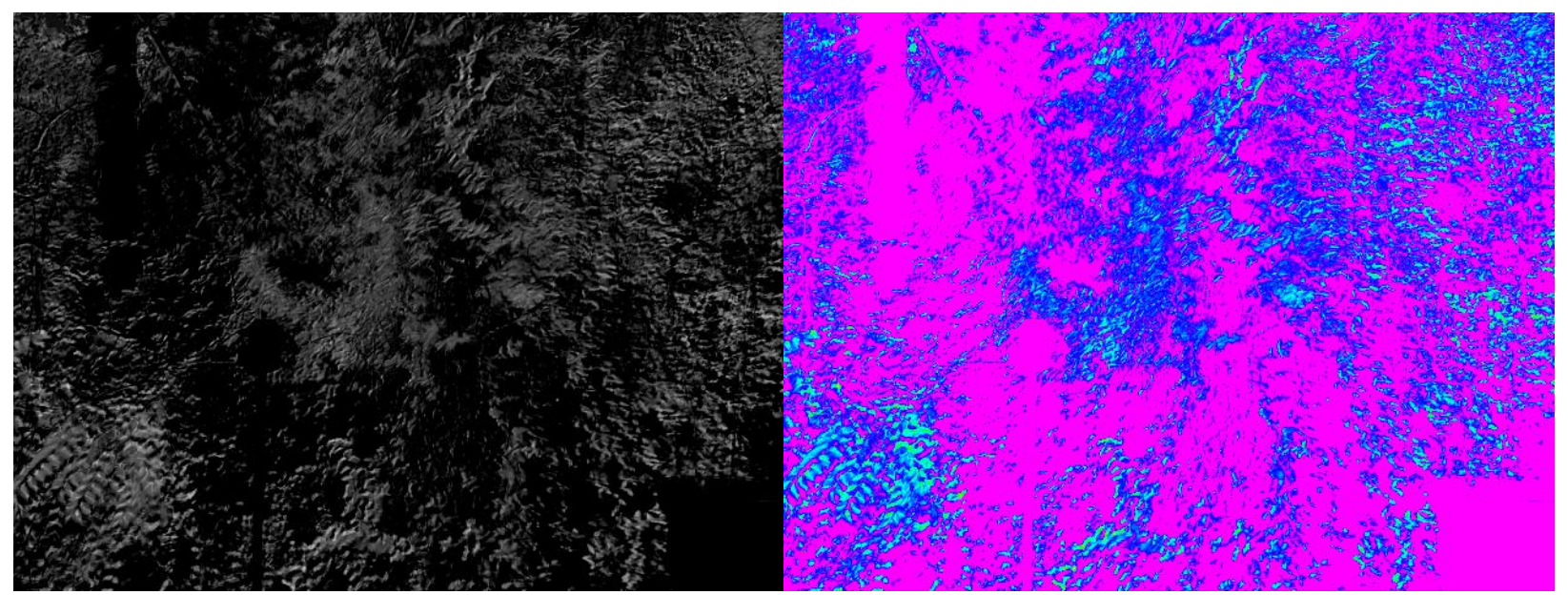

Figure 54: Raw NDVI (left) and NDVI Color Map (right) of Landscape Still Shot with Blue Filter 


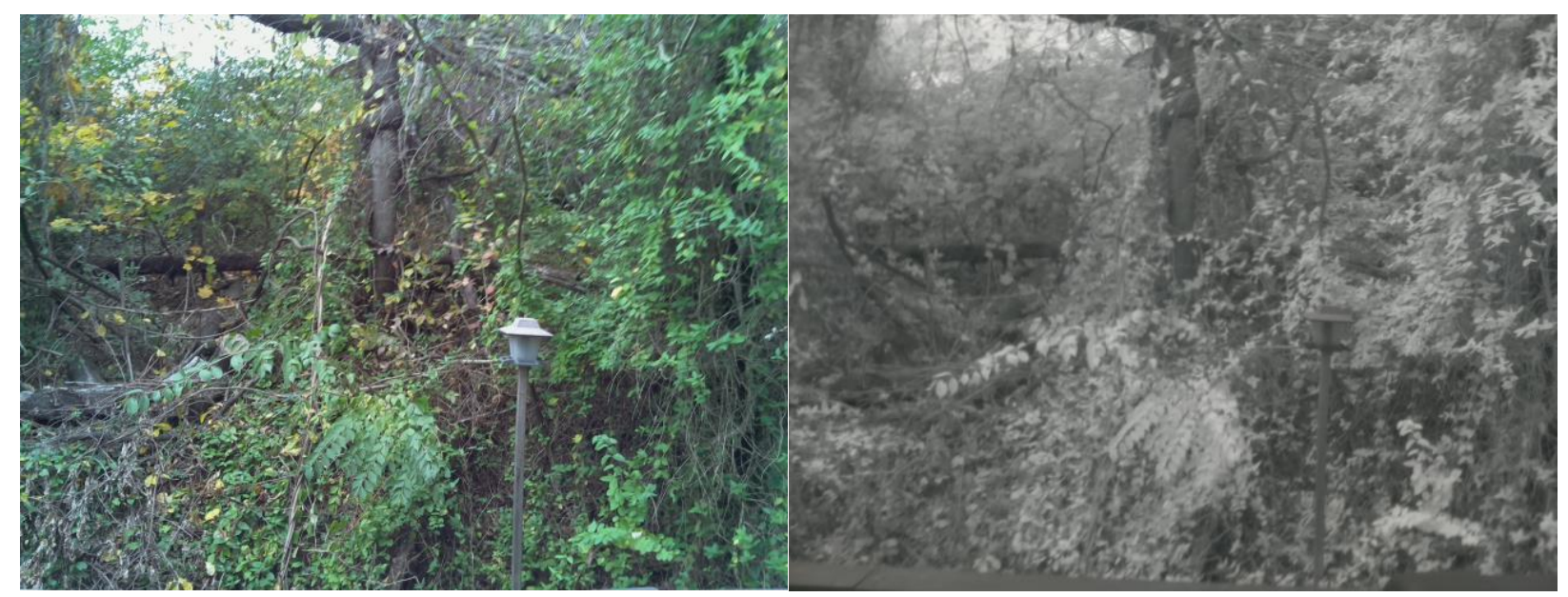

Figure 55: Original RGB (left) and NIR (right) Images of Landscape Still Shot with Black Filter

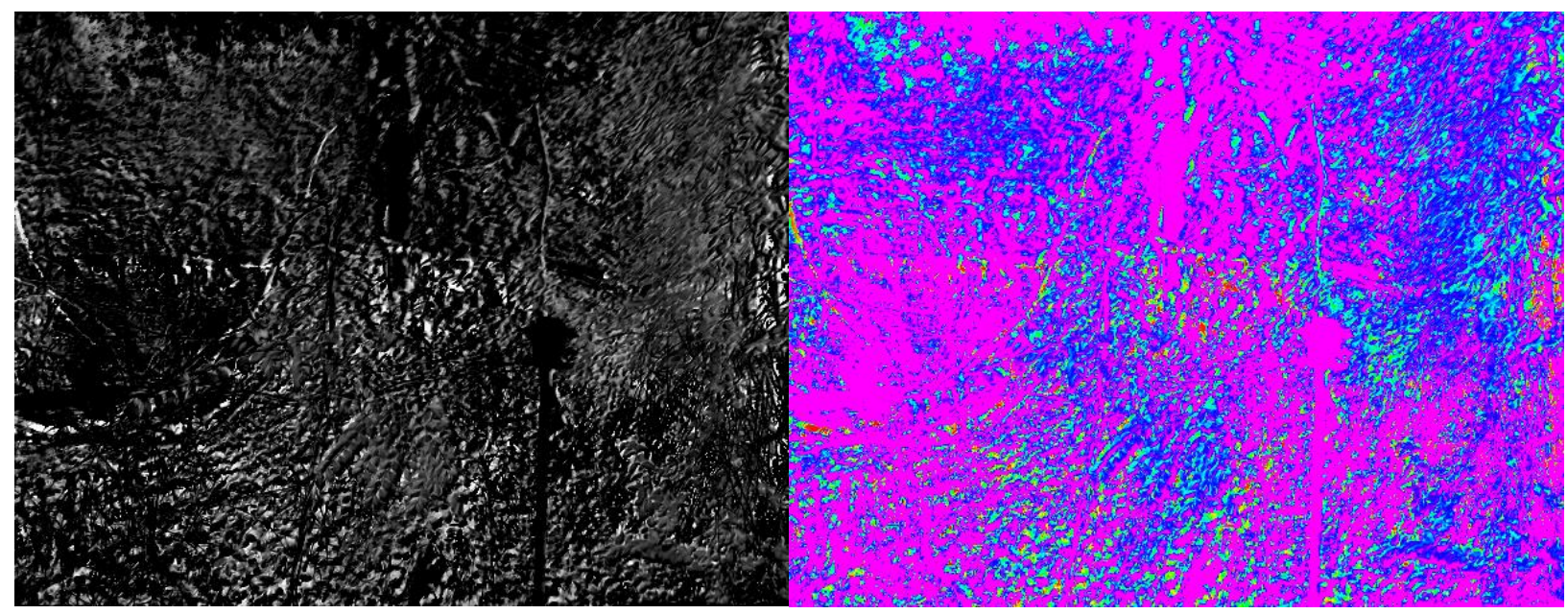

Figure 56: Raw NDVI (left) and NDVI Color Map (right) of Landscape Still Shot with Black Filter

\subsection{Flight Testing}

\subsubsection{UAV Flight}

Initial flight testing was performed using a small COTS quadcopter UAV called the Parrot AR.Drone. This UAV platform used for testing was somewhat undersized for longer flights to collect substantial NDVI data. The photographs in Figure 57 illustrate the size of the quadcopter relative to the NDVI payload and how it was attached to the airframe. The overall size of the Parrot AR.Drone airframe is approximately 1 square foot with 5" diameter propellers. 


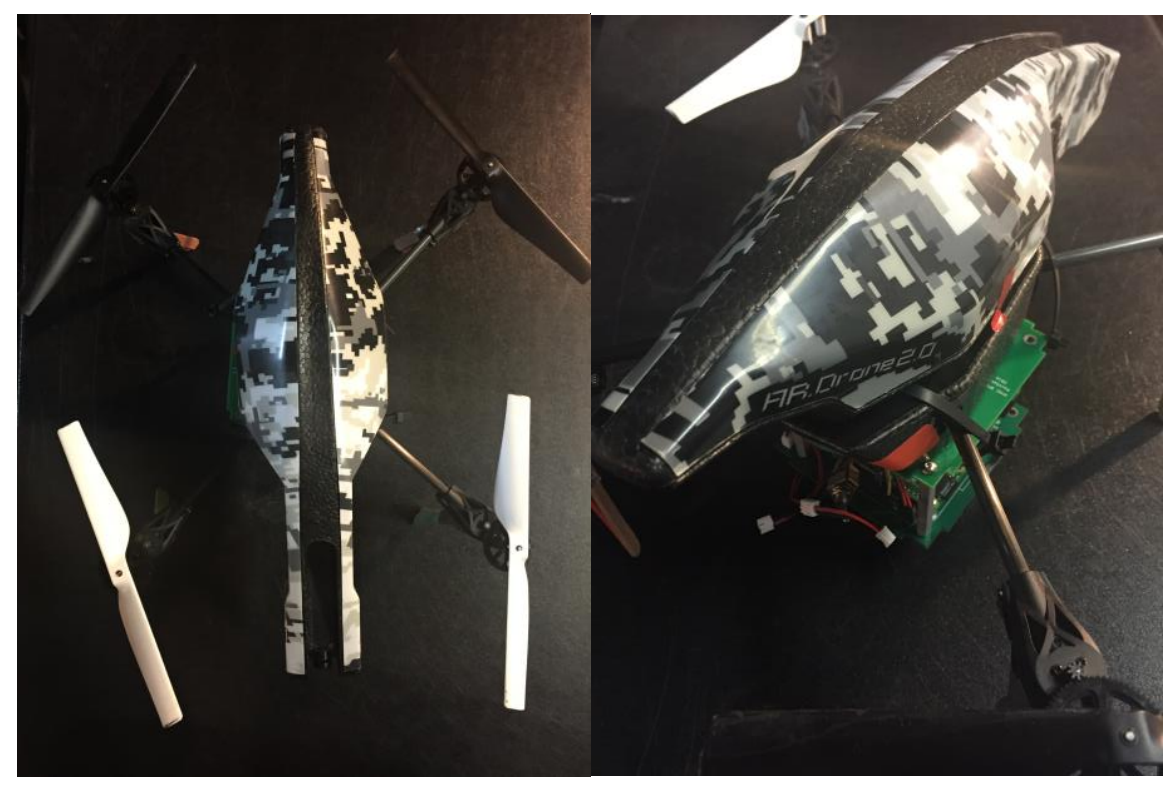

Figure 57: NDVI Payload Attached to the Parrot AR.Drone Quadcopter

Since the Parrot AR.Drone quadcopter has a built-in camera for in-flight photography, it was not designed for carrying additional payload packages. The control system struggled to maintain stability with the added payload package and observed flight times were only five minute or less on a single battery compared to 10-15 minutes without the payload. Images were collected from stabilized flight at approximately $15 \mathrm{ft}$ AGL over a grass lawn in a suburban neighborhood in Sabraton, WV and are shown in Figure 58. The raw NDVI and NDVI color map follow in Figure 59. It can be seen from the NDVI analysis that in order to align the images, the NIR image had to be slightly translated and rotated overtop of the RGB image. This is mainly due to the slight delay in the image capture between the two cameras as the UAV quadcopter happened to be rotating (yawing). Results of the NDVI analysis suggest that this section of the lawn may need some additional seed planted in the spring as there appears to be several bare spots in the lawn. 


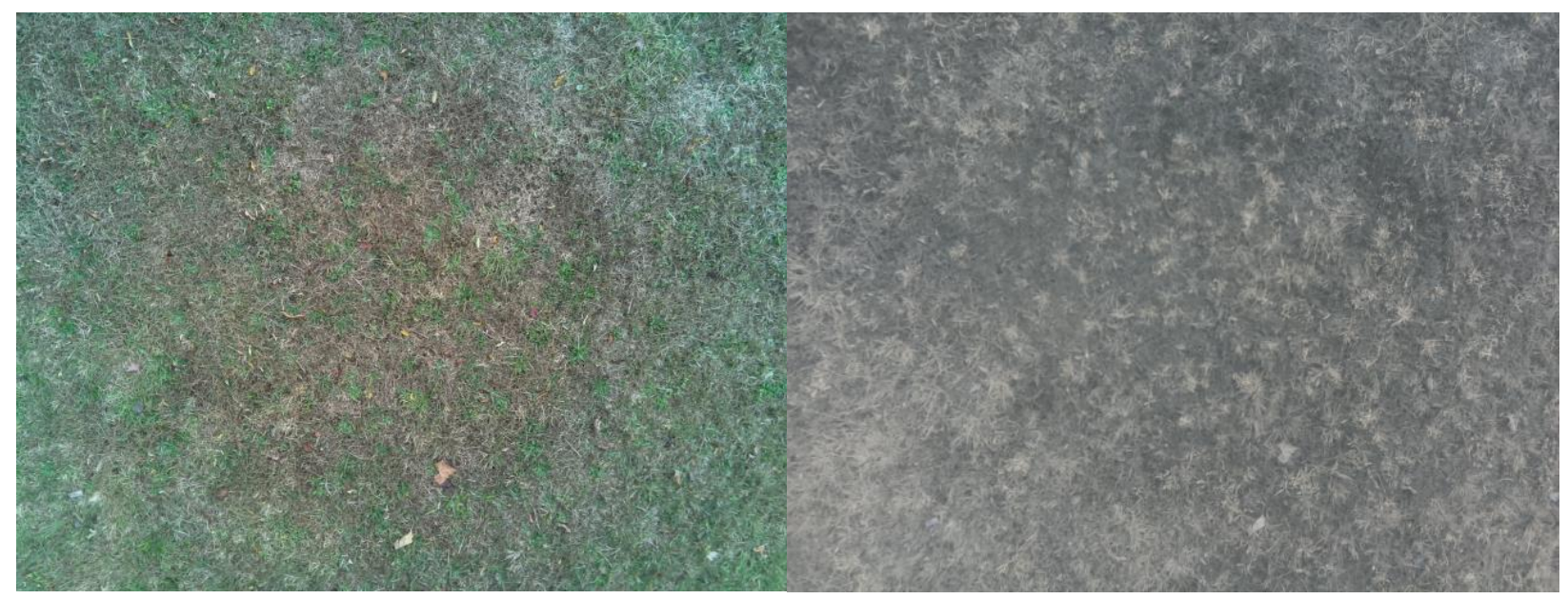

Figure 58: Original RGB (left) and NIR (right) Images of Grassy Area Taken with UAV at $10 \mathrm{ft}$ AGL

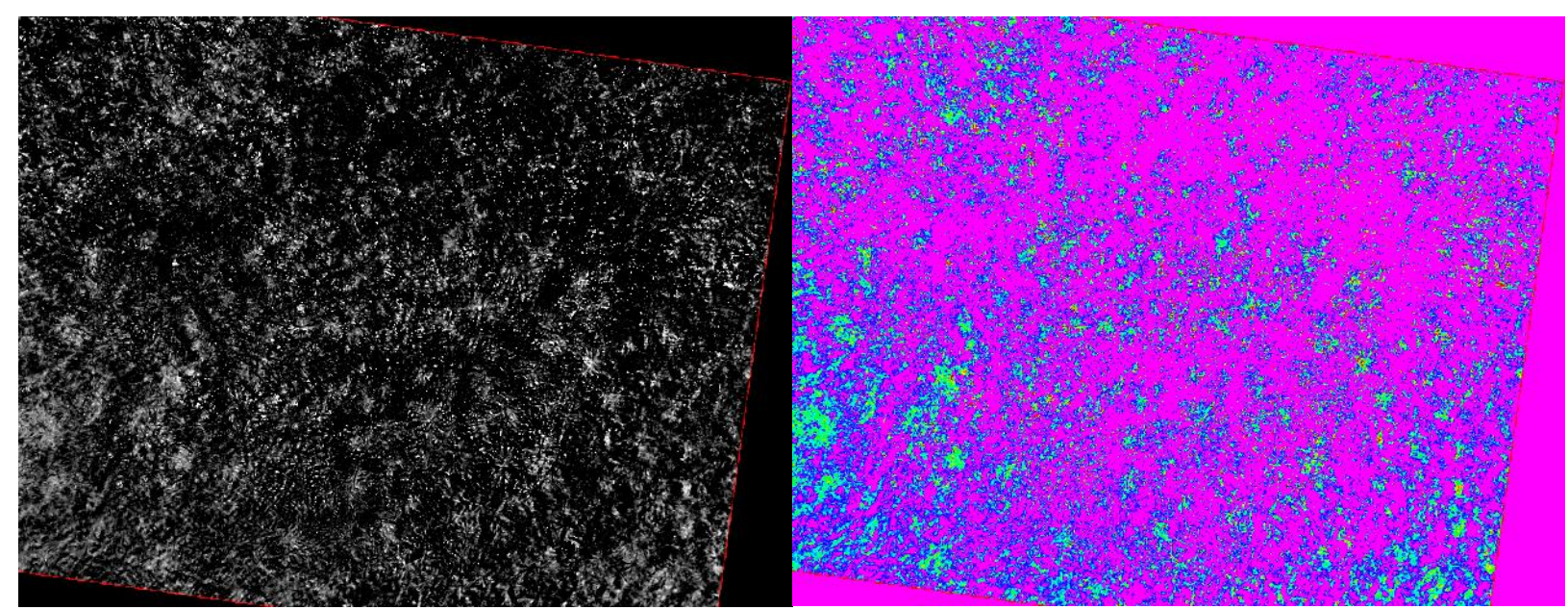

Figure 59: Raw NDVI (left) and NDVI Color Map (right) of Grassy Area Taken with UAV at $10 \mathrm{ft}$ AGL

\subsubsection{General Aviation Flight}

The main flight testing of the NDVI Cubesat/UAV payload was performed on a single engine, general aviation aircraft (Cessna 152) flight over the Cheat Lake area outside of Morgantown, WV. This flight test was done at the end of autumn when most of the trees had either lost their leaves or were changing colors due to vegetation senescence and abscission. The flight takeoff time was approximately 9:30 AM EST. The atmospheric conditions were overcast with intermittent sunshine. Photos captured from the runway during takeoff and directly following takeoff are shown in Figure 60. These images were included to show the type of aircraft used during this test flight and the relative size of the airport. 


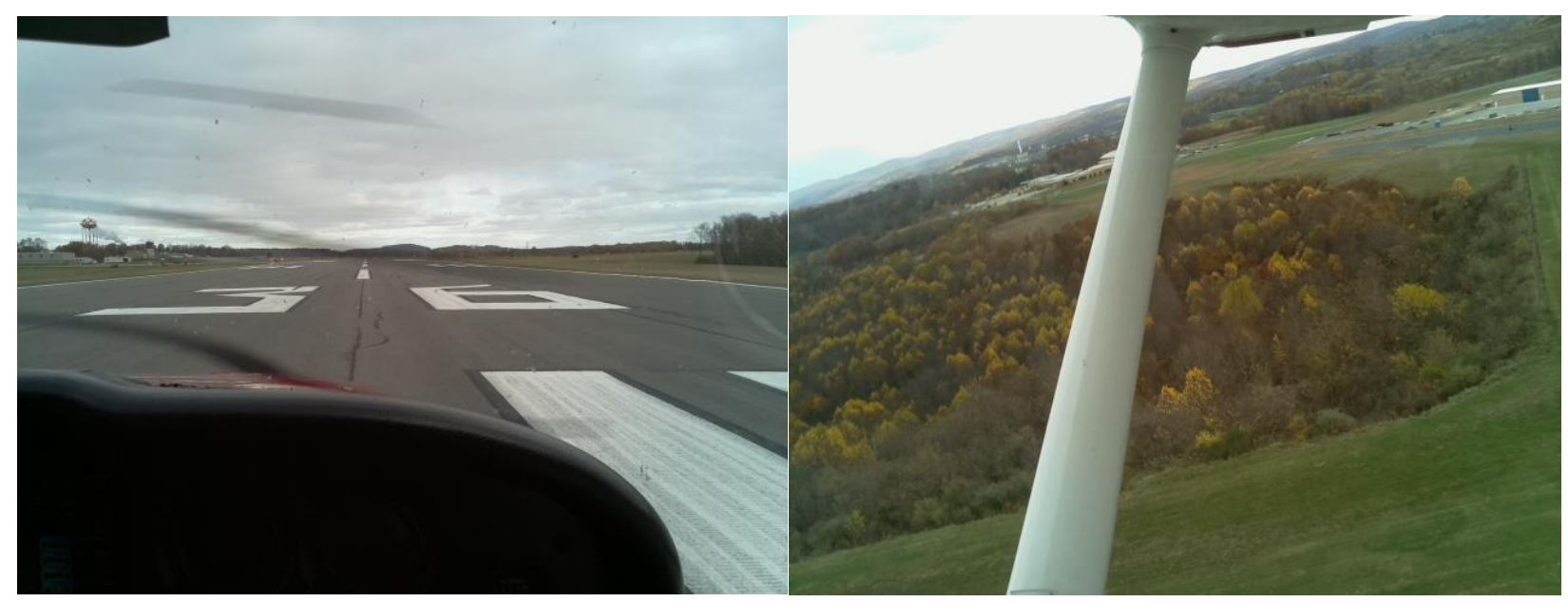

Figure 60: RGB Photos Taken during Takeoff in a Cessna 152 General Aviation Airplane

Several pairs of RGB and NIR images were captured at various altitudes as the pilot was instructed to fly along the ridgeline of Chestnut Ridge in Northern WV. In Figure 61, images were captured from approximately $250 \mathrm{ft}$ AGL while the aircraft was still climbing up to flight pattern altitude above the runway. The scene captured in these photos included a wood line with an open patch of grassland. The resulting raw NDVI image and NDVI color map are shown in Figure 62. In this figure, the greener sections of the grasslands show up as lighter areas on the raw NDVI image and green/yellow colors on the color map. The red spots indicate the highest amount of photosynthetic activity.

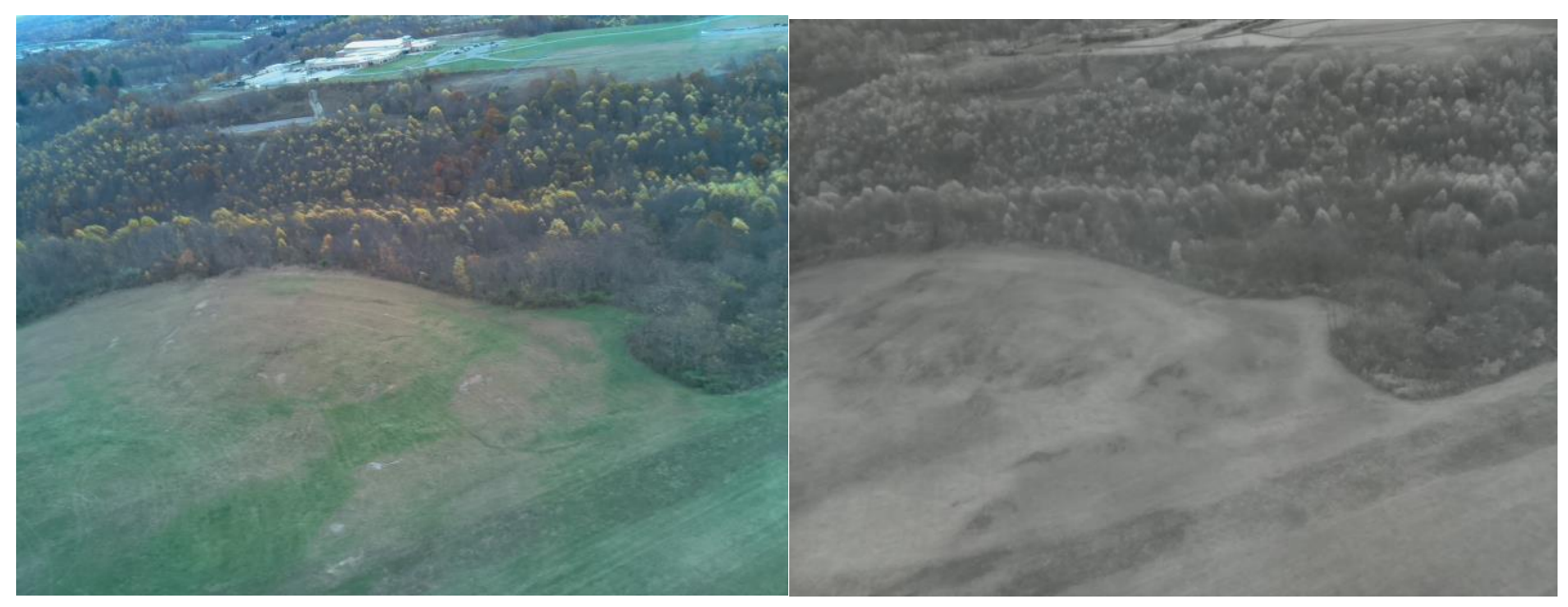

Figure 61: Original RGB (left) and NIR (right) Images of Woodline and Field Taken with General Aviation Aircraft at $250 \mathrm{ft}$ AGL 


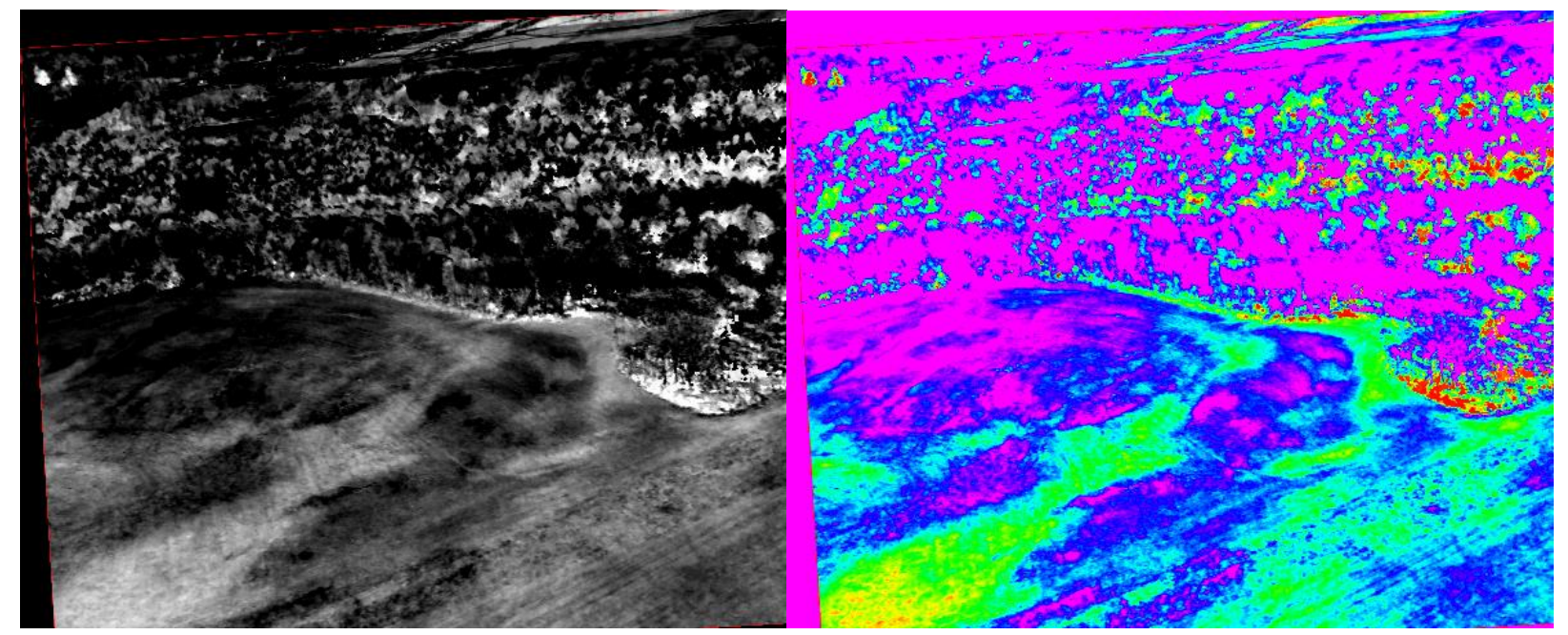

Figure 62: Raw NDVI (left) and NDVI Color Map (right) of Woodline and Field Taken with General Aviation Aircraft at $250 \mathrm{ft}$ AGL

As the aircraft approached $500 \mathrm{ft}$ AGL, another scene was captured showing the treetops of Chestnut Ridge. These images are shown in Figure 63. The raw NDVI and NDVI color map at $500 \mathrm{ft}$ AGL above the ridgeline can be seen in Figure 64. These results suggest that most of the photosynthetic activity of the deciduous trees has become dormant for the upcoming winter season.

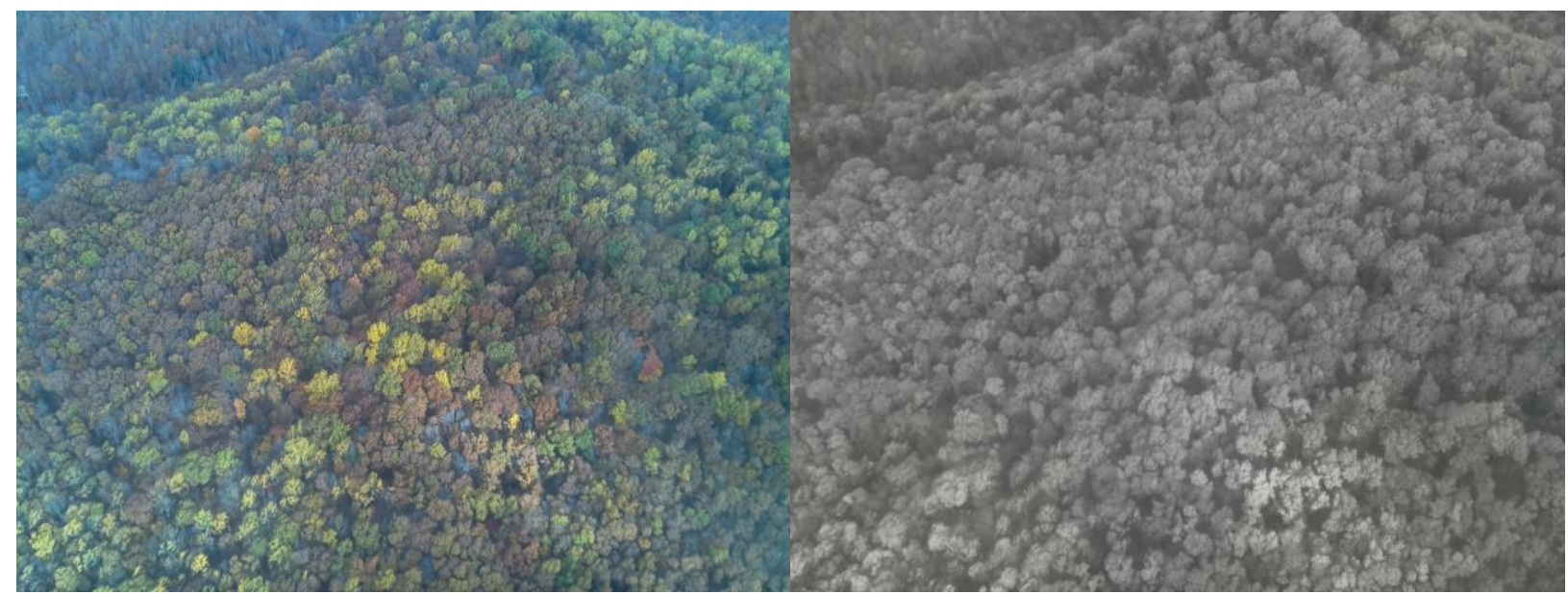

Figure 63: Original RGB (left) and NIR (right) Images of Ridgeline Treetops with General Aviation Aircraft at $500 \mathrm{ft}$ AGL 


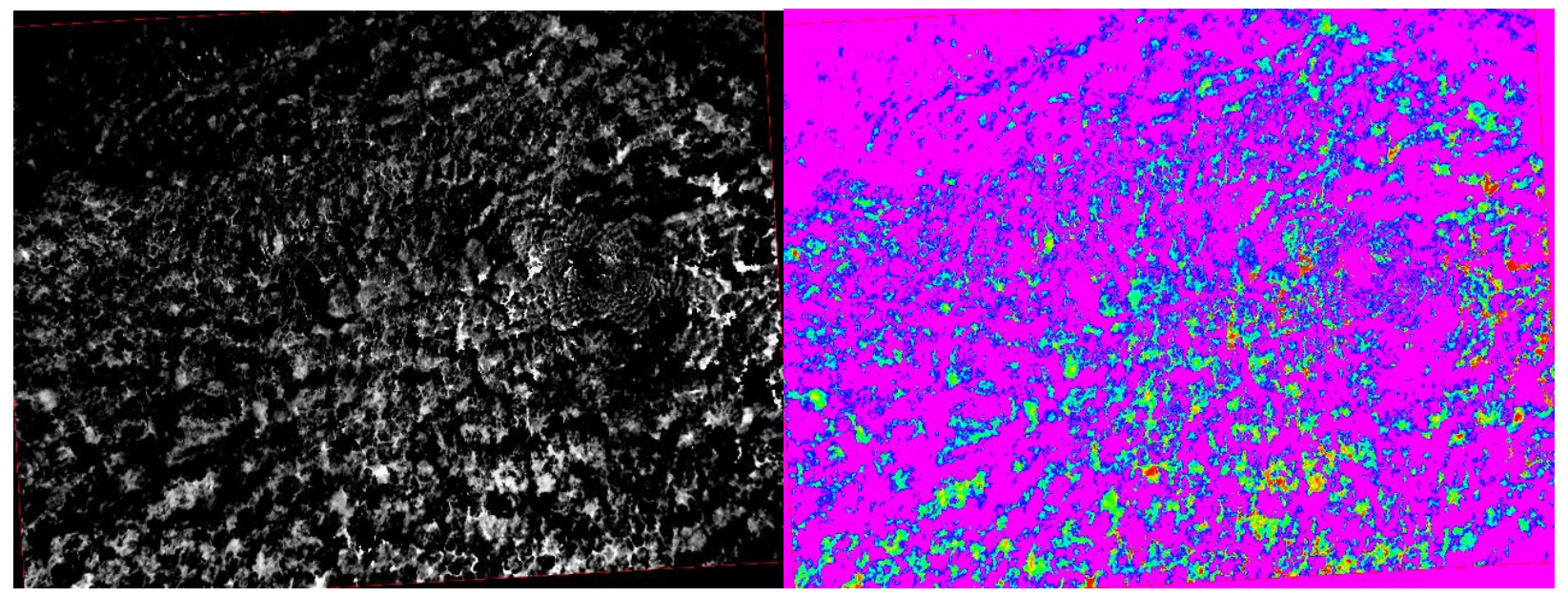

Figure 64: Raw NDVI (left) and NDVI Color Map (right) of Ridgeline Treetops with General Aviation Aircraft at 500 ft AGL

The next pair of images was captured above the ridgeline showing the treetops at an altitude of approximately $1000 \mathrm{ft}$ AGL. These images are presented in Figure 65. The resulting raw NDVI and NDVI color map for this pair of images are presented in Figure 66. The results from this scene also suggest that most of the photosynthetic activity of the leaves on the trees has become dormant with only a few areas of red and yellow. There are somewhat more areas that show up as green and blue which indicate some active vegetation but not as healthy as the areas that are red or yellow areas.

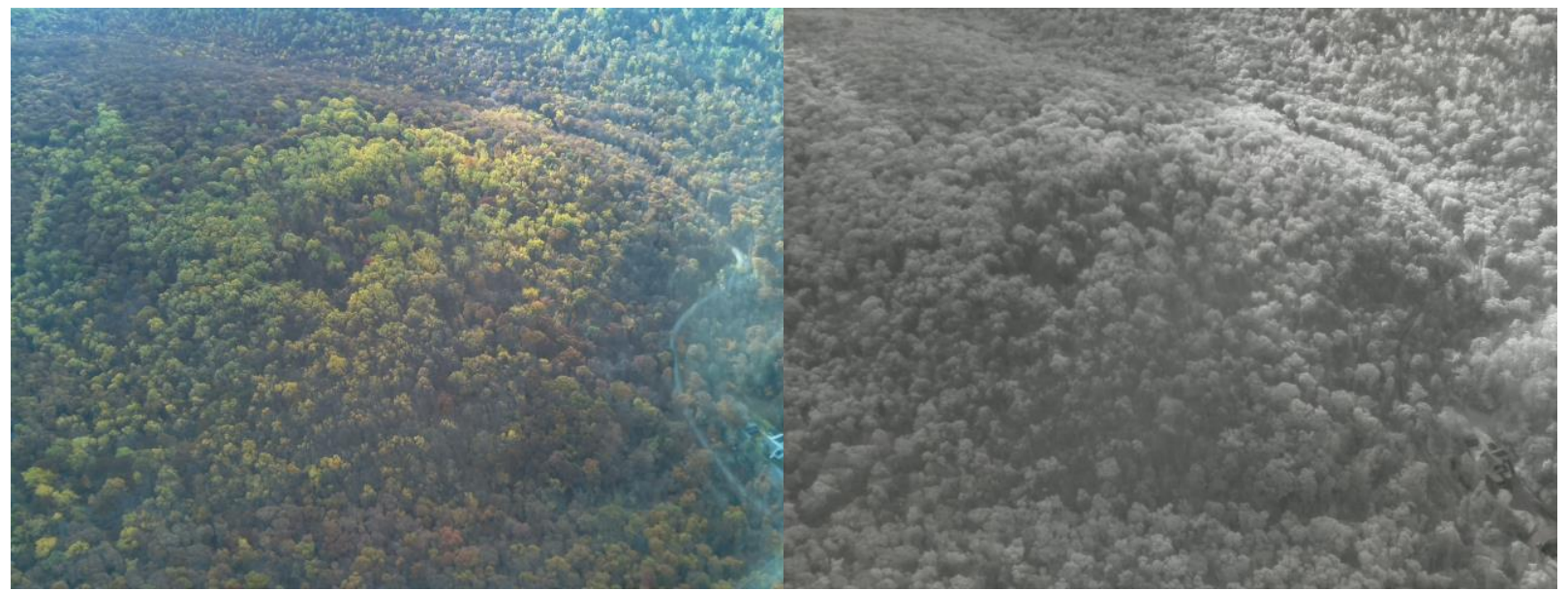

Figure 65: Original RGB (left) and NIR (right) Images of Ridgeline Treetops with General Aviation Aircraft at 1000 ft AGL 


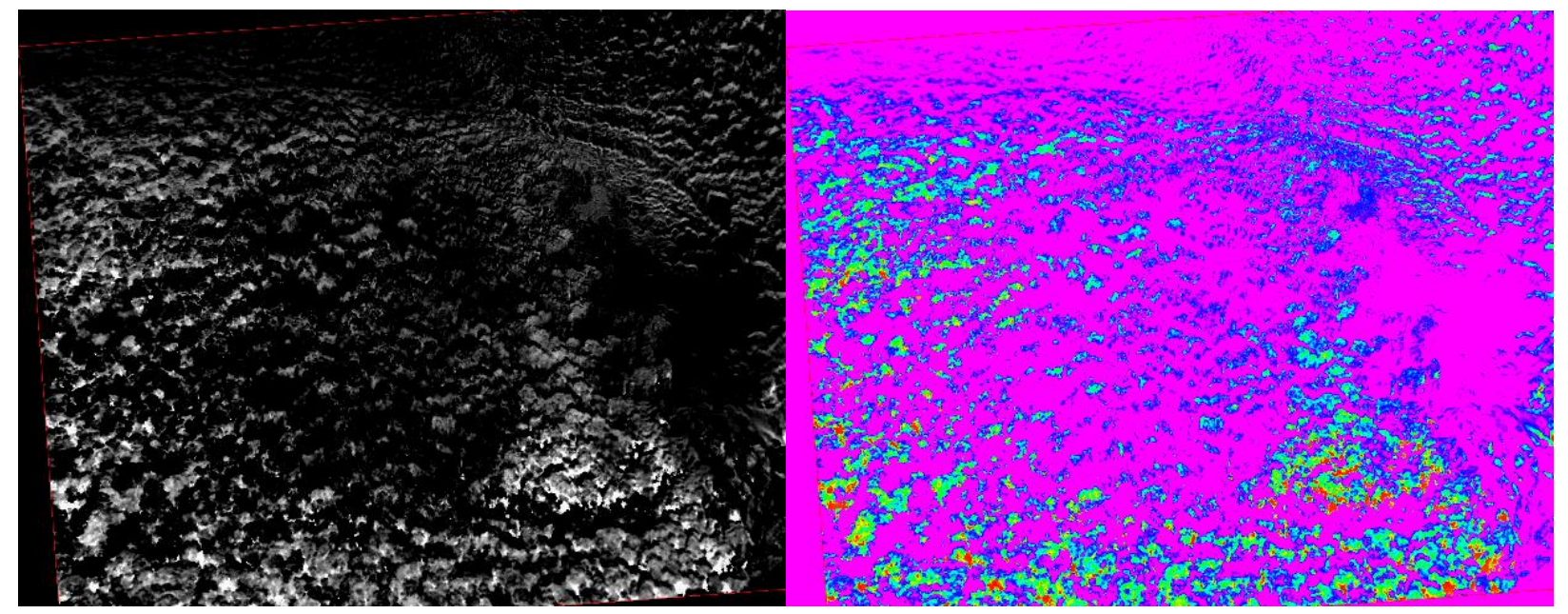

Figure 66: Raw NDVI (left) and NDVI Color Map (right) of Ridgeline Treetops with General Aviation Aircraft at $1000 \mathrm{ft}$ AGL

As the aircraft continued to gain altitude, the next pair of images captured a local rock quarry in the image frame. These images were taken at an altitude of $2000 \mathrm{ft}$ AGL and are shown in Figure 67 followed by the resulting raw NDVI and NDVI color map images in Figure 68. Since rocks do not absorb visible light as do plants and the NIR light is reflected almost the same as visible light is reflected, the NDVI value resulting from the rock surface is very low and is represented by the magenta color. This is similar to the sky or to bodies of water, tree bark, and other non-photosynthetic objects. Another interesting area can be seen in the lower-left quadrant of the NDVI color map shown in Figure 68. An area of healthy vegetation is shown in red surrounding a pond. It makes sense that the vegetation surround this water source is healthier than the rest of the trees outside the immediate perimeter of the pond.

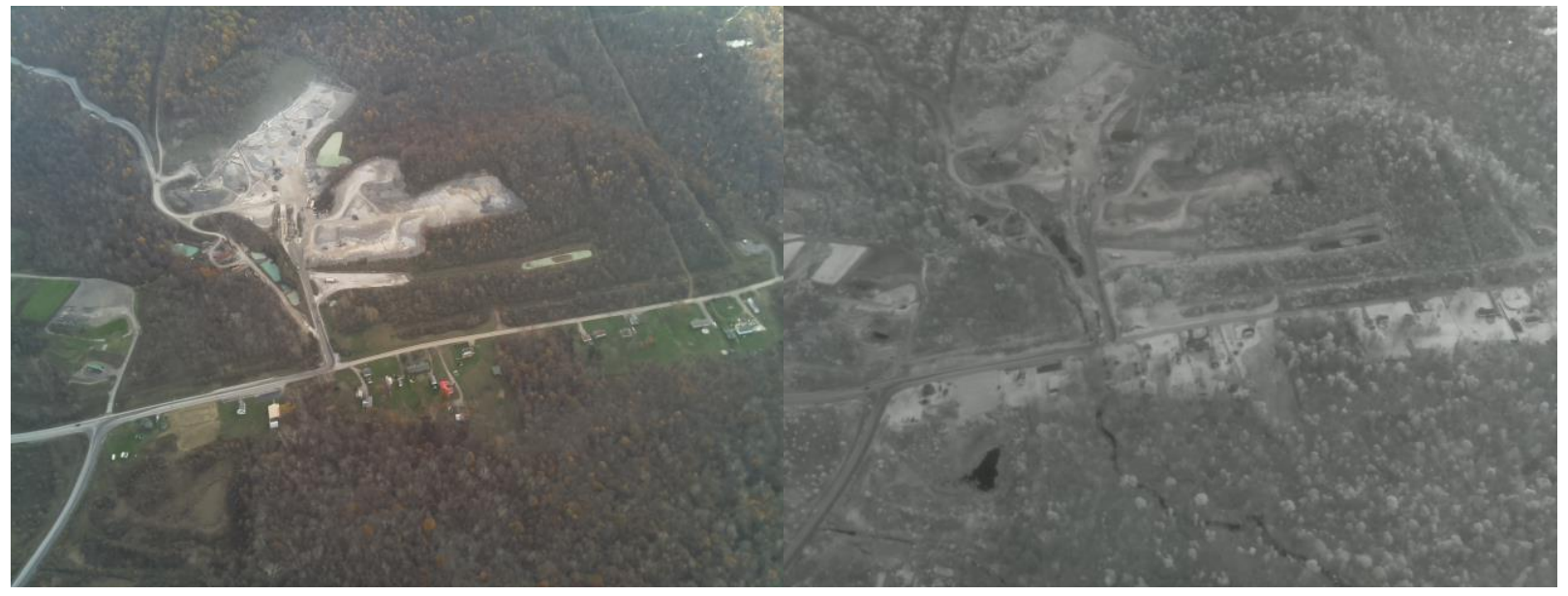

Figure 67: Original RGB (left) and NIR (right) Images of Rock Quarry with General Aviation Aircraft at $2000 \mathrm{ft}$ AGL 


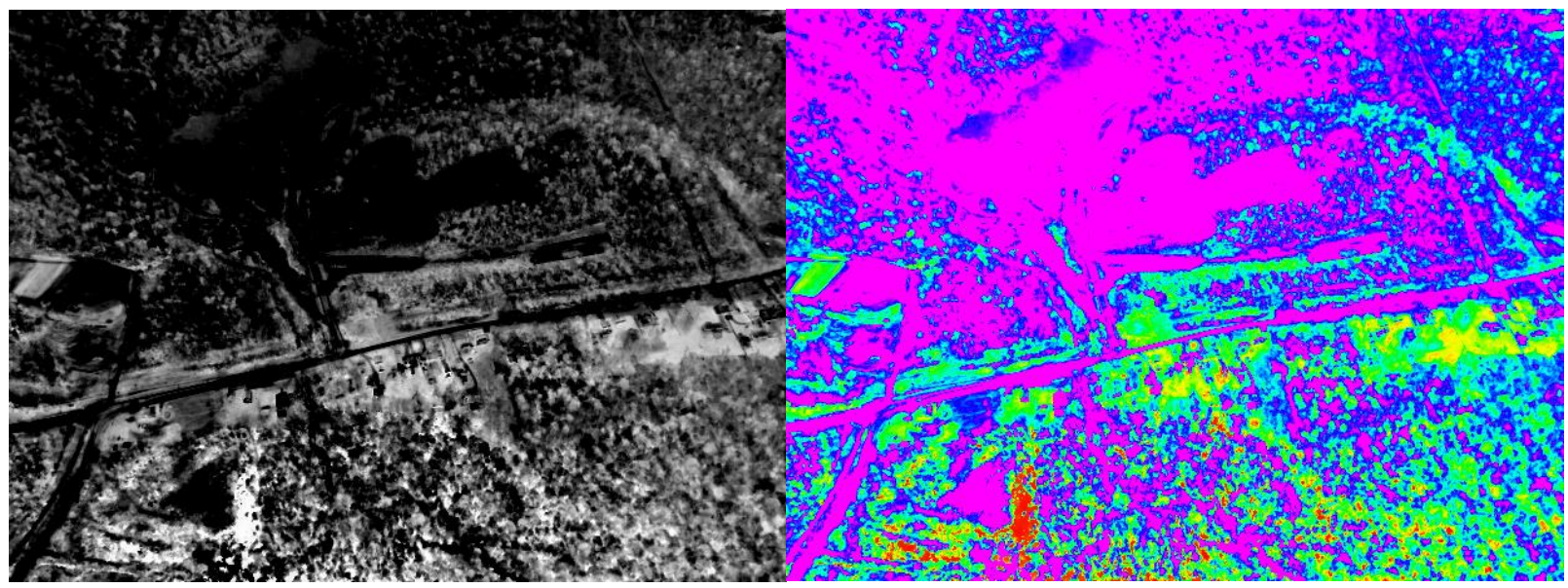

Figure 68: Raw NDVI (left) and NDVI Color Map (right) of Rock Quarry with General Aviation Aircraft at 2000 ft AGL

Another pair of images was captured while flying at $2000 \mathrm{ft}$ AGL that shows the ridgeline with open fields on both sides of the ridge. The RGB and NIR images are displayed in Figure 69. The resulting raw NDVI and color map images are displayed in Figure 70, where the open fields show up as a blue or aqua color. An area of red showing up on the left portion of the color map image shows an area of healthier vegetation which is most likely a creek bed where overflow may trickle from the pond nearby. As in the other photos, most of the trees do not appear to have level of photosynthesis occurring during the end of autumn when these images were captured.

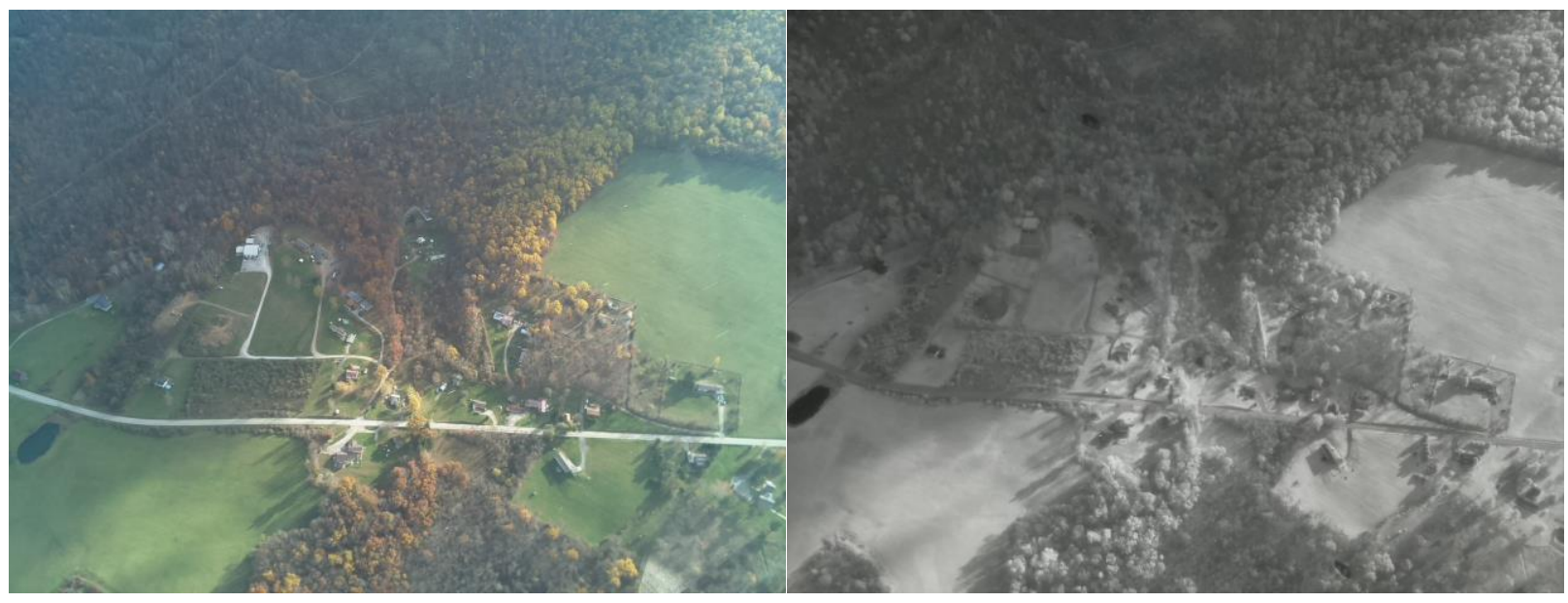

Figure 69: Original RGB (left) and NIR (right) Images of Ridgeline and Field with General Aviation Aircraft at 2000 ft AGL 


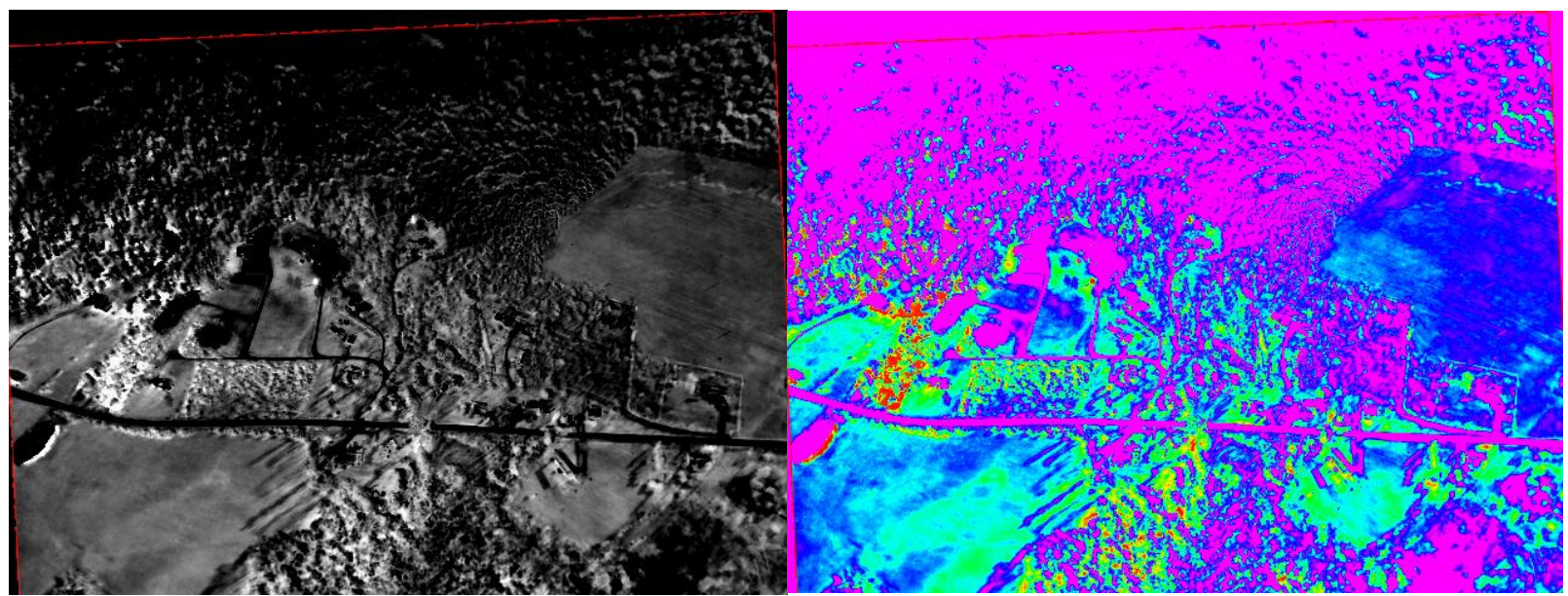

Figure 70: Raw NDVI (left) and NDVI Color Map (right) of Ridgeline and Field with General Aviation Aircraft at $2000 \mathrm{ft}$ AGL

The final images captured during the general aviation aircraft flight testing were at an altitude of approximately $3000 \mathrm{ft}$ AGL. The cloud ceiling on the day of the flight testing was at about $4500 \mathrm{ft}$ above sea level (ASL). Since the Morgantown airport is positioned at about $1200 \mathrm{ft}$ ASL, this means the aircraft could only fly up to a maximum of about $3000 \mathrm{ft}$ AGL flying by visual flight rules (VFR). The next pair of images captured during the flight test was pointed at a larger angle of incidence to the ground in effort to capture Cheat Lake in the horizon. The original RGB and NIR images can be seen in Figure 71 while the resulting NDVI raw/color map images are illustrated in Figure 72.

This resulting NDVI distribution appears to be directly proportional to the angle of incidence of the light reflected off the treetops and onto the camera lenses of the NDVI payload. The treetops closer to the bottom of the image yield a higher NDVI value indicating areas of healthier vegetation whereas the treetops in the middle appear to be less healthy and the treetops near the top of the image are shown to have almost no photosynthetic activity at all. The lake area and the very top of the image should yield very low values of NDVI which makes sense because water reflects nearly all ambient light off of it and the horizon is shining ambient light. This results in nearly equal intensity values of visible light and NIR light on pixels corresponding to the water and horizon which produces a value near zero in the numerator of Equation (1) and therefore an overall NDVI value close to zero. However, higher values of NDVI were expected for the trees directly boarding the lake area due to the additional water supply. The angle of incident being higher for the section of the image where the lake is position could explain this counter-intuitive result. Furthermore, it can be clearly seen in Figure 71 that clouds were blocking a large portion of the sunlight in this aerial scene. Toward the middle of the right side of the image, there is an area of sunlight penetrating the clouds and illuminating the treetops in the area. This resulted in a low NDVI value which was the opposite of what was expected, however this could truly be due to patches of dead or dormant trees in that area. Other areas of penetrating sunlight near the top and along the edges of the image produce areas of 
higher NDVI values as expected due to the additional sunlight reflected by the treetops. It should be noted that there happened to be a slight glare on the aircraft window that showed up in the image overtop of the lake area which was disregarded in the NDVI analysis. Similar to the light collected from the horizon, the glare produced an NDVI value near zero due to the subtraction of nearly equal intensity values of NIR and VIS light in the numerator of the NDVI equation.

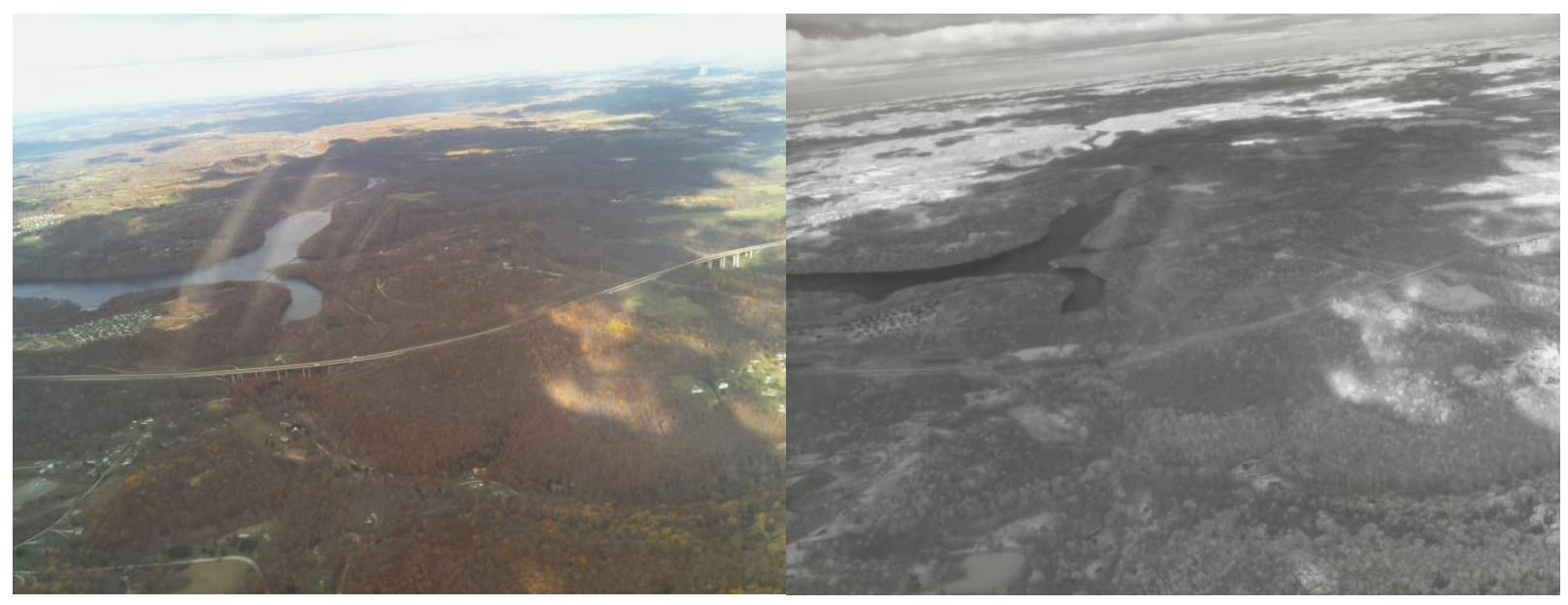

Figure 71: Original RGB (left) and NIR (right) Images of Cheat Lake with General Aviation Aircraft at $3000 \mathrm{ft}$ AGL

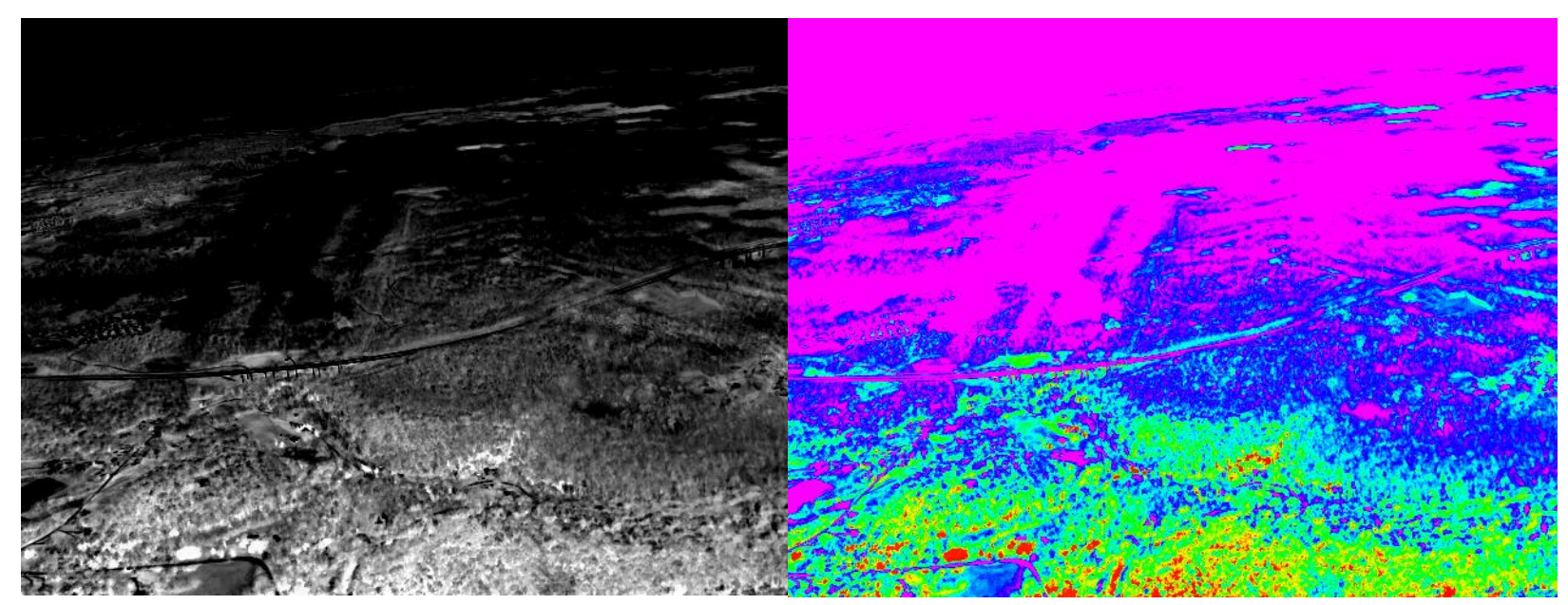

Figure 72: Raw NDVI (left) and NDVI Color Map (right) of Cheat Lake with General Aviation Aircraft at $3000 \mathrm{ft}$ AGL

The final pair of images captured by the experimental NDVI payload was taken at $3000 \mathrm{ft}$ AGL along the ridgeline with a more direct angle down on the treetops. The original images are provided in Figure 73 and Figure 74 illustrates the NDVI results of these images. The color distributions of the leaves on the trees in the RGB image are directly proportional to the resulting NDVI value. For instance, a band of senescent yellow leaves stretches from the center of the image over to the left edge. The resulting NDVI color map shows this area as a band of magenta 
which indicates a low value of NDVI or vegetation mortality. This makes sense because the leaves are ready to die and fall off due to the abscission process of plant physiology. Similarly, the greener treetops yield larger NDVI values shown by the blue, green and even some areas of red indicating higher levels of vegetation growth. The right side of the image became washed out from the lack of sunlight on that side of the ridgeline due to the shadowing effect of the morning sun.

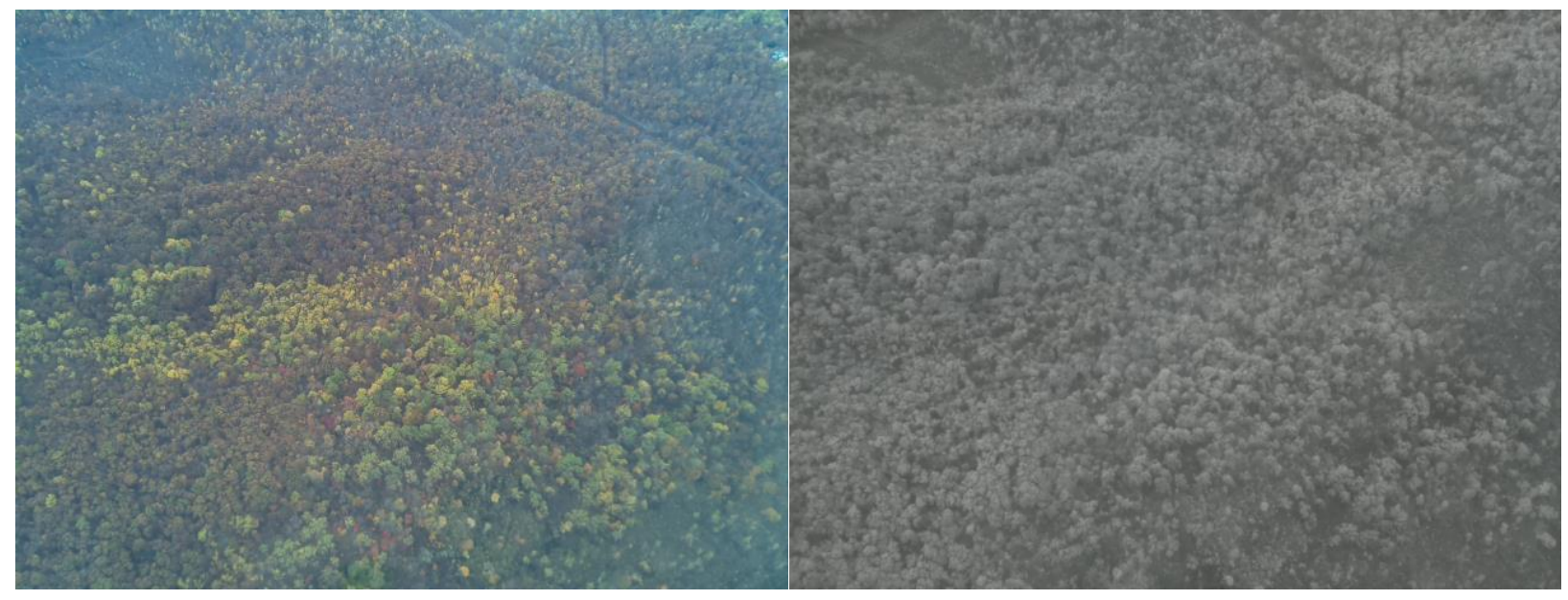

Figure 73: Original RGB (left) and NIR (right) Images of Ridgeline with General Aviation Aircraft at $3000 \mathrm{ft}$ AGL

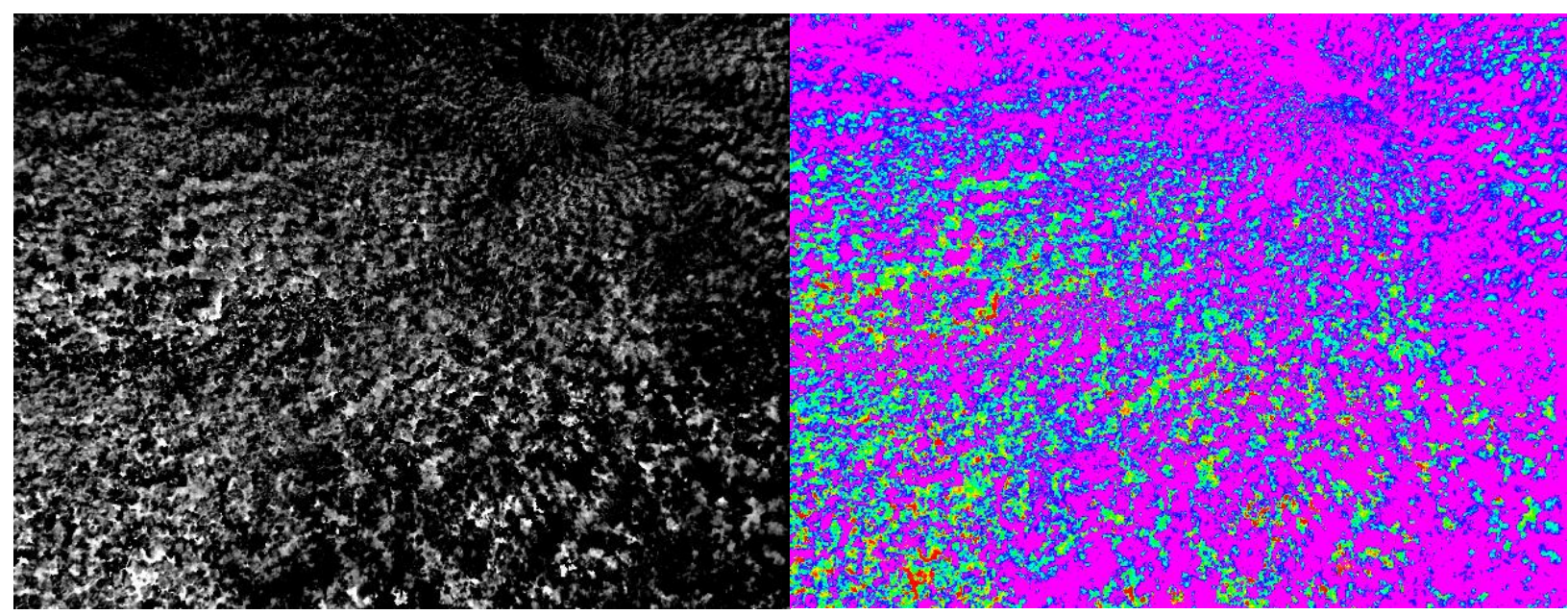

Figure 74: Raw NDVI (left) and NDVI Color Map (right) of Ridgeline with General Aviation Aircraft at $3000 \mathrm{ft}$ AGL 


\section{Chapter 5 | Conclusions and Recommendations}

\subsection{Summary}

The proposed experimental payload provides a low-cost, open-source software solution with a simple commercial off-the-shelf (COTS) hardware design for NDVI analysis from low altitude to upper atmosphere and space applications. Flight tests conducted with the NDVI Cubesat/UAV payload reinforce the feasibility of the low-cost payload concept for remotelysensed earth sciences. The raw NDVI values collected during these flight experiments can potentially provide useful information for other scientists and technologist to determine whether the proposed payload could be used as solution to enable or enhance their research efforts. Programs that are developing new technology and embarking in the field of space sciences and UAV applications may also consider using this NDVI payload configuration as a baseline for their own missions in the future.

To increase the effectiveness of the NDVI results, it has been verified that test data should be gathered at times when the sun is directly overhead or with a solar altitude of at least $35^{\circ}$ relative to the horizon. Therefore, in the northern hemisphere testing should be performed roughly within the hours of $10 \mathrm{AM}$ and $2 \mathrm{PM}$ between the vernal and autumnal equinox. To optimize the results of the NDVI analysis, research should be done to determine the local time of maximum solar altitude for particular dates and locations of interest, and tests should be performed as close to that time as possible. The test results presented in this research showed areas of shadowing produced by the ridgeline with the sun relatively low in the sky only a few hours after the break of sunrise. These areas cause false mapping of NDVI values due to the absence of sunlight. Also, the inherent delay between the two cameras caused slight offsets between the respective images captured. This offset was corrected using GIMP software to align the images, however it is recommended for future prototypes to consider the use of the RPi Compute Module which employs two on-board camera slots and may reduce the delay between image capture of the two camera modules thereby increasing maximum framerate. The use of two on-board camera slots would also reduce code complexity and external wiring. However, this would more than double the cost of the payload from about $\$ 100$ to $\$ 225$ so these improvements must be weighed with the increase of cost for the application at hand. The main increase in cost is due to the development board required by the RPi Compute module. If more than one payload is to be produced, the cost would only increase slightly over the proposed $\$ 100$ payload for additional builds after the first because the RPi Compute development board can be reused. This also comes with added electrical design complexity as the ribbon cable connections for each camera slot would need to be incorporated into the PCB design if the RPi Compute module is implemented without the development board. Slot connectors for the RPi Compute Module would also have to be incorporated in the payload PCB design in order to reuse the RPi Compute development board. 
In summary, a small, low-cost NDVI payload has been designed to fit the Cubesat platform and built with light weight components to allow for flights on smaller quadcopter UAV airframes and for extended operations on larger class UAVs. Based on the extensive literature study performed on current Cubesat development efforts, a science payload that mimics the proposed payload as not been found. The results have proven the practicality of this unique payload design for use in academia and other areas of developing technologies and capabilities. The data analysis provided in this thesis also corroborates other claims that NDVI data should be gathered within a specified range of solar altitudes. Finally, PCB designs, schematics, and opensource software have been provided to aid others in continued use and advancement of the proposed NDVI payload.

\section{$5.2 \quad$ Future Work}

Although the $8 \mathrm{MP}$ cameras used in this payload provided suitable results from the various altitudes tested in flight, it is almost guaranteed that evolvements in COTS technology will produce an improved version of the optical sensors embedded in the camera modules which will capture images with even higher definition. Since the quality of the results are directly related to the camera resolution, it is highly recommended for future missions utilizing this payload configuration to take advantage of these advancement in technology and upgrade the camera modules with the latest versions as they are released. Also, the addition of a $45^{\circ}$ beam splitting mirror to the camera lenses will also reduce post-processing complexities by eliminating the need to manually align the visible and near-infrared images prior to generating the NDVI results.

The method of applying this NDVI analysis can be extended to remote sensing on a larger scale though high-altitude balloon flights, suborbital rocket launches, and orbital cubesat missions. An IOS Cubesat Kit has been partially assembled and a fit check was done to show that the NDVI payload meets the requirements for the cubesat platform. Photographs of the payload integration with the IOS Cubesat kit are shown in Figure 75. For orbital missions, the addition of an array of light sensors should be considered to only take pictures when the sun is shining at a low angle of incidence so that valuable downlink time is not wasted sending unwanted images back to the user ground station. Future plans for the addition of a forwardlooking infrared (FLIR) sensor for thermal imaging will further expand the NDVI payload applications to include ocean temperature and ice sheet topography mapping. 


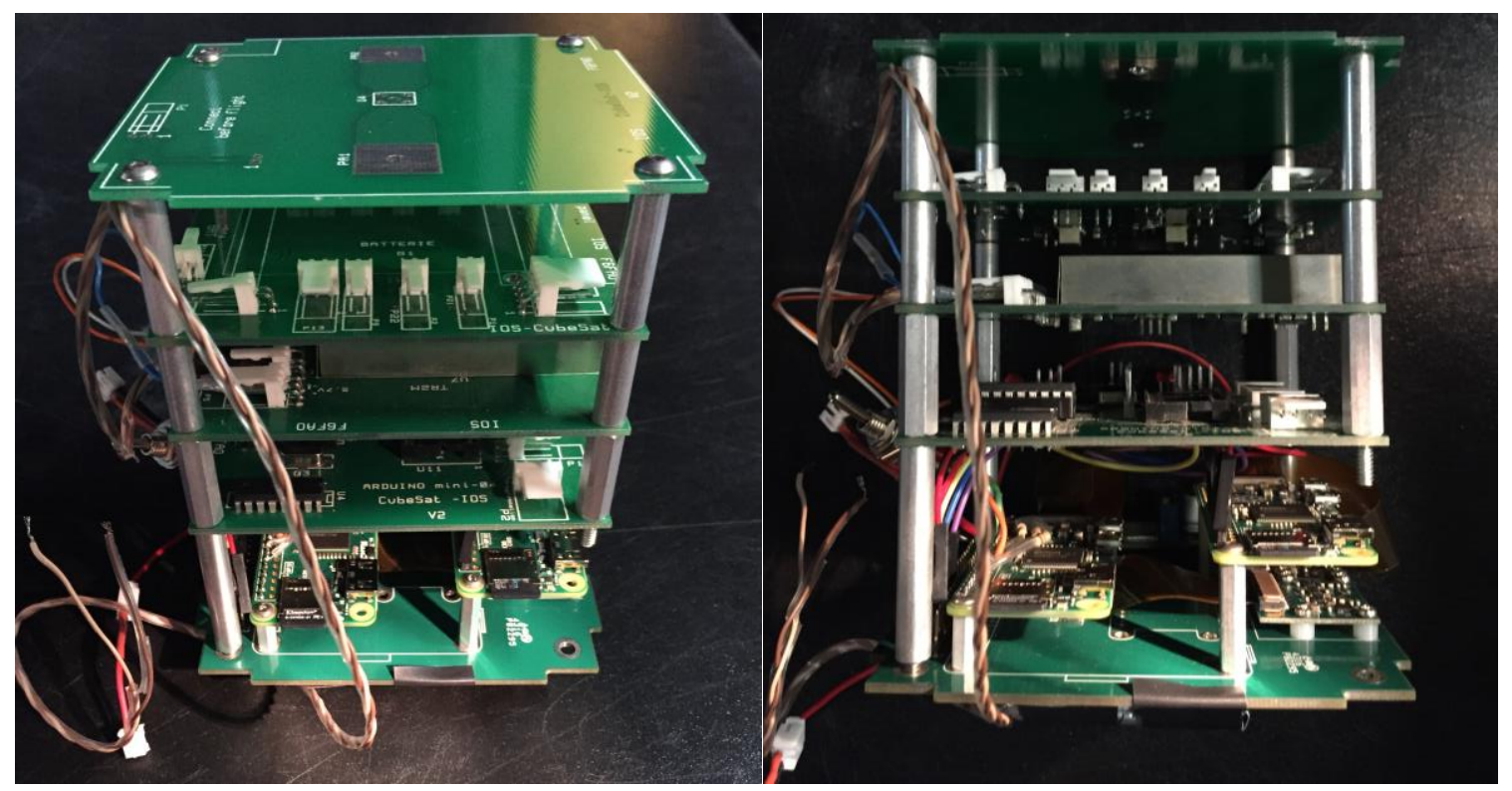

Figure 75: NDVI Payload Integration with IOS CubeSat Kit

\subsection{Lessons Learned}

The incidence angle of sunlight reflected from the area of interest is a concern that seems to affect the results of NDVI analysis. Higher angles of incidence could cause increasing error in the reflectance intensities from both the RGB and NIR cameras. At lower altitudes, these errors can be further amplified with terrain through effects of shadowing. Scheduling image capture times when the sun is nearly overhead will provide consistency and added confidence in the integrity of the data collected by the science payload. Also with the addition of a thermal camera and light sensors for future orbital missions, scheduling NDVI during the sunlight and FLIR imaging during shadow could provide a practical and reasonable solution for data collection.

The type of filter used for the NIR camera was also determined to be an important factor. Although some NDVI analysis can be done using the blue filter, greater definition was observed when the black filter was applied. Also, it was determined through initial testing that the autoexposure function of the digital camera modules seemed to affect the first few images captured. Therefore, the delay between images was placed prior to the capture of the image to allow additional time for the camera module to adjust to the brightness of the scene it is capturing. 


\section{References}

[1] J. Gillan. (2013, Jan 31). Normalized Difference Vegetation Index. The Landscape Toolbox [Online]. (2/15). Available:

http://wiki.landscapetoolbox.org/doku.php/remote sensing methods:normalized difference vegetation index

[2] John Weier and David Herring. (2000, Aug 30). Measuring Vegetation: Normalized Difference Vegetation Index (NDVI). NASA Earth Observatory [Online]. (2/4). Available:

http://earthobservatory.nasa.gov/Features/MeasuringVegetation/measuring vegetation 2.php

[3] Jesslyn F. Brown. (2015, Jan 12). NDVI, the Foundation for Remote Sensing Phenology. USGS Remote Sensing Phenology: Vegetation Indices [Online]. Available:

https://phenology.cr.usgs.gov/ndvi foundation.php

[4] U. Meier, H. Bleiholder, H. Brumme, E. Bruns, B. Mehring, T. Proll, and J. Weigand. (2009, Aug). Phenological Growth Stages of Roses. Annals of Applied Biology [Online]. 154(2), pp.231-238. Available:

https://doi.org/10.1111/j.1744-7348.2008.00287.x

[5] P. Munger, H. Bleiholder, H. Hack, M. Hess, R. Stauss, T. Boom, and E. Weber. Phenological Growth Stages of the Soybean Plant (Glycine Max L. MERR.): Codification and Description According to the BBCH Scale. Journal of Agronomy and Crop Science [Online]. 179(4), pp. 209-217. Available:

https://doi.org/10.1111//.1439-037X.1997.tb00519.x

[6] Michael Schultz, Jan Clevers, Sarah Carter, Jan Verbesselt, Valerio Avitabile, Hien Vu Quang, and Martin Herold. Performance of Vegetation Indices from Landsat Time Series in Deforestation Monitoring. International Journal of Applied Earth Observations and Geoinformation [Online]. 52, pp. 318-327. Available:

https://doi.org/10.1016/i.jag.2016.06.020

[7] John Weier and David Herring. (2000, Aug 30). Measuring Vegetation: NDVI as an Indicator of Drought. NASA Earth Observatory [Online]. (3/4). Available:

http://earthobservatory.nasa.gov/Features/MeasuringVegetation/measuring vegetation 3.php

[8] A. AghaKouchak, A. Farahmand, F. S. Melton, J. Teixeira, M. C. Anderson, B. D. Wardlow, and C. R. Hain. (2015, Jun 29). Remote Sensing of Drought: Progress, Challenges and Opportunities. Reviews of Geophysics [Online]. 53(2), pp. 452-480. Available: http://onlinelibrary.wiley.com/doi/10.1002/2014RG000456/epdf

[9] Xin Cao, Xihong Cui, Miao Yue, Jin Chen, Hiroki Tanikawa, and Yu Ye. (2013, Jun 19). Evaluation of Wildfire Propagation Susceptibility in Grasslands Using Burned Areas and Multivariate Logistic Regression. International Journal of Remote Sensing [Online]. 34(19), pp. 6679-6700. Available:

https://doi.org/10.1080/01431161.2013.805280

[10] Samson Reiny, (2017, Jan 9). NASA Study Finds a Connection Between Wildfires and Drought. NASA Earth Science News [Online]. Available:

https://www.nasa.gov/feature/goddard/2017/nasa-study-finds-a-connection-between-wildfires-and-drought

[11] A.R. Huete and R.D. Jackson. (1987, Nov). Suitability of Spectral Indices for Evaluating Vegetation Characteristics on Arid Rangelands. Remote Sensing of Environment [Online]. 23(2), pp. 213-232. Available: https://doi.org/10.1016/0034-4257(87)90038-1

[12] Genesis T. Yengoh, David Dent, Lennart Olsson, Anna Tengberg, and Compton J. Tucker. Use of the Normalized Difference Vegetation Index (NDVI) to Assess Land Degradation at Multiple Scales: Current Status, Future Trends, and Practical Considerations. (SpringerBriefs in Environmental Science) [Online]. Cham, Switzerland: Springer, 2015. Available: https://doi.org/10.1007/978-3-319-24112-8

[13] Jackson, Thomas J., Daoyi Chen, Michael Cosh, Fuqin Li, Martha Anderson, Charles Walthall, Paul Doriaswamy, and E.Ray Hunt. 2004. Vegetation Water Content Mapping Using Landsat Data Derived Normalized Difference Water Index for Corn and Soybeans. Remote Sensing of Environment [Online]. 92(4) pp. 475-482. Available: https://doi.org/10.1016/i.rse.2003.10.021

[14] Isaac C. Rhew, Ann Vander Stoep, Anne Kearney, Nicholas L. Smith, and Matthew D. Dunbar. (2011, Dec 1). Validation of the Normalized Difference Vegetation Index as a Measure of Neighborhood Greenness. Annals of Epidemiology [Online]. 21(12), pp. 946-952. Available: https://doi.org/10.1016/i.annepidem.2011.09.001

[15] Jakob Bro-Jørgensen, Molly Brown, and Nathalie Pettorelli. (2008, Aug 1). Using the Satellite-Derived Normalized Difference Vegetation Index (NDVI) to Explain Ranging Patterns in a Lek-Breeding Antelope: the Importance of Scale".Oecologia [Online]. 158(1), pp. 177-182. Available: https://doi.org/10.1007/s00442-008-1121-z 
[16] Jean-Yves Pontailler, Graham J. Hymus, and Bert G. Drake. (2003). Estimation of Leaf Area Index Using GroundBased Remote Sensed NDVI Measurements: Validation and Comparison with Two Indirect Techniques. Canadian Journal of Remote Sensing [Online]. 29(3), pp.381-387. Available: https://doi.org/10.5589/m03-009

[17] K. Soudani, et al. (2012, Aug). "Ground-Based Network of NDVI Measrements for Tracking Temporal Dynamics of Canopy Structure and Vegetation Phenology in Different Biomes". Remote Sensing of Environment [Online]. 123, pp. 234-245. Available: https://doi.org/10.1016/.irse.2012.03.012

[18] Gerassimos G. Peteinatos, Martin Weis, Dionisio Andújar, Victor Rueda Ayala, and Roland Gerhards. (2013, Dec 6). Potential Use of Ground-Based Sensor Technologies for Weed Detection. Pest Management Science [Online]. 70(2), pp: 190-199. Available: http://onlinelibrary.wiley.com.www.libproxy.wvu.edu/doi/10.1002/ps.3677/epdf

[19] Victor V Klemas. (2015, Apr 13). Coastal and Environmental Remote Sensing from Unmanned Aerial Vehicles: An Overview. Journal of Coastal Research [Online]. 31(5), pp. 1260-1267. Available: https://doi.org/10.2112/JCOASTRES-D-15-00005.1

[20] Kevan B. Moffett and Steven M. Gorelick. (2012, Oct 10). Distinguishing Wetland Vegetation and Channel Features with Object-Based Image Segmentation. International Journal of Remote Sensing [Online]. 34(4), pp. 1332-1354. Available: https://doi.org/10.1080/01431161.2012.718463

[21] John V. Stafford. Precision Agriculture '13. (Proceedings of the 9Th European Conference on Precision Agriculture) [Online]. Catalonia, Spain: Wageningen Academic Publishers, 2013. Available: http://dx.doi.org/10.3920/978-90-8686-778-3

[22] J.A. Shaw, et al. (2012, Oct 15). Multispectral Imaging System on Tethered Balloons for Optical Remote Sensing Education and Outreach. Proceedings of Society of Photo-Optical Instrumentation Engineers [Online]. 8481(Optics Education and Outreach II, 84810T). Available: http://dx.doi.org/10.1117/12.932236

[23] Kamel Didan. (2017, Sept 5). Vegetation Indices. MODIS Land [Online]. Available: https://modisland.gsfc.nasa.gov/vi.html

[24] Lynn Jenner. (2017, Aug 3). Landsat Overview. Landsat [Online]. (1/4). Available: https://www.nasa.gov/mission pages/landsat/overview/index.html

[25] USGS Land Remote Sensing Program. Effects of Drought. Land Remote Sensing Image Collections [Online]. (1/202). Available: https://remotesensing.usgs.gov/gallery/gallery.php?cat=2\#284

[26] Daniel Selva and David Krejci. (2012, Feb 1). A Survey and Assessment of the Capabilities of Cubesats for Earth Observation. Acta Astronautica [Online]. 74(7-8), pp. 50-68. Available: https://doi.org/10.1016/..actaastro.2011.12.014

[27] A.S. Driesman. (2011, Jun 30). Developing Young Space System Engineers. IEEE Aerospace and Electronic Systems Mag [Online]. 26(6). pp. 4-13. Available: https://doi.org/10.1109/MAES.2011.5936180

[28] Tassia Owen. (2017, Nov 22). Mission. Terra - EOS Flagship [Online]. (2/8). Available: https://terra.nasa.gov/about/mission

[29] Steve Graham. (2017, Nov 2). Aqua Earth-observing Satellite Mission. Aqua Project Science [Online]. Available: https://aqua.nasa.gov/

[30] Heather Hanson. (2017, Mar 31). NASA Earth Science Division Operating Missions. NASA's Earth Observing System Project Science Office [Online]. Available: https://eospso.gsfc.nasa.gov/

[31] Huang J, Dai Q, Wang H, and Han D. (2014, Aug 5). Analysis of Ndvi Data for Crop Identification and Yield Estimation. IEEE Journal of Selected Topics in Applied Earth Observations and Remote Sensing [Online]. 7(11) pp. 4374-4384. Available: https://doi.org/10.1109/JSTARS.2014.2334332

[32] Andrew N. Yuhas and Louis A. Scuderi. (2008, Nov 28). MODIS-Derived NDVI Characterisation of DroughtInduced Evergreen Dieoff in Western North America". Geographical Research [Online]. 47(1). pp. 34-45. Available: http://onlinelibrary.wiley.com/doi/10.1111/.1745-5871.2008.00557.x/full

[33] X. Li and G. Shao. (2014, Nov 14). Object-Based Land-Cover Mapping with High Resolution Aerial Photography at a County Scale in Midwestern USA. Remote Sensing [Online]. 6(11). pp. 11372-11390. Available:

http://dx.doi.org/10.3390/rs61111372

[34] Kirk Woellert, Pascale Ehrenfreund, Antonio J. Ricco, and Henry Hertzfeld. (2010, Oct 14). Cubesats: CostEffective Science and Technology Platforms for Emerging and Developing Nations. Advances in Space Research [Online]. 47(4). pp. 663-684. Available: https://doi.org/10.1016/i.asr.2010.10.009

[35] Erik Kulu. (2017, Nov 18). Nanosatellite \& Cubesat Database. [Online]. Available: http://www.nanosats.eu/ 
[36] Michael Swartwout. (2009, Apr 3). The first one Hundred University-Class spacecraft 1981 -2008. IEEE Aerospace and Electronic Systems Magazine [Online]. 24(3). pp. A1-A24. Available:

https://doi.org/10.1109/MAES.2009.4811089

[37] Justin Morris, Matthew Grubb. (2015, May 11). Meet STF-1. [Online] (1/7). Available: http://stf1.com

[38] Justin Morris, Matthew Grubb. (2015, May 11). West Virginia University Research. [Online] (4/7). Available: http://stf1.com/research.php

[39] Shanessa Jackson. (2017, Aug 3). CubeSat Launch Initiative. NASA [Online]. (1/5). Available: https://www.nasa.gov/directorates/heo/home/CubeSats_initiative

[40] Michael Asbury. (2017, Aug 24). Simulation to Flight 1. NASA IV\&V Program [Online]. Available: https://www.nasa.gov/centers/ivv/istar/stf1.html

[41] Michael Asbury. (2017, Aug 3). Space Flight Design Challenge. NASA IV\&V Program [Online]. Available: https://www.nasa.gov/centers/ivv/sfdc.html

[42] Michael Asbury. (2017, Aug 3). OC-Flight-1. NASA IV\&V Program [Online]. Available: https://www.nasa.gov/centers/ivv/jstar/ocflight1.html

[43] Randa Milliron. (2017, Nov 6). IOS TubeSat Kits. Interorbital Systems [Online]. Available: http://interorbital.com/Tubesat\%20Kits

[44] Randa Milliron. (2017, Nov 6). IOS CubeSat Kits. Interorbital Systems [Online]. Available: http://interorbital.com/Cubesat\%20Kits

[45] Texas Instruments. (2015, Sep) LM2731 0.6/1.6-MHz Boost Converters With 22-V Internal FET Switch in SOT-23. Datasheet [Online]. Available: http://www.ti.com/lit/ds/symlink/lm2731.pdf

[46] Raspberry Pi Foundation. (2017, Sep 8). NOOBS. [Online]. Available: https://www.raspberrypi.org/downloads/noobs/

[47] The GIMP Team. (2017, May 11). GIMP GNU Image Manipulation Program. [Online]. Available: https://www.gimp.org/ 


\section{Appendix A: Preliminary Assessment of Cubesat Utility}

\begin{tabular}{|c|c|c|c|c|}
\hline Parameter & $\begin{array}{l}\text { Selected measurement } \\
\text { concepts }\end{array}$ & $\begin{array}{l}\text { Utility comparted to } \\
\text { traditional } \\
\text { architectures } \\
\text { (comparable, lower, } \\
\text { marginal) }\end{array}$ & $\begin{array}{l}\text { Cubesat technology } \\
\text { readiness (flight } \\
\text { proven, in } \\
\text { development, } \\
\text { unavailable) }\end{array}$ & $\begin{array}{l}\text { Scientific readiness } \\
\text { (mature, } \\
\text { exploratory, } \\
\text { conceptual) }\end{array}$ \\
\hline Aerosols & $\begin{array}{l}\text { Uncooled miniature } \\
\text { spectrometers (VNIR, with } \\
\text { polarimetry and/or multi- } \\
\text { angular measurements }\end{array}$ & Lower & In development & Exploratory \\
\hline $\begin{array}{l}\text { Atmospheric } \\
\text { chemistry (ozone } \\
\text { and trace gases) }\end{array}$ & $\begin{array}{l}\text { Uncooled minature } \\
\text { spectrometers (UV + SWIR) }\end{array}$ & Lower & In development & Mature \\
\hline $\begin{array}{l}\text { Atmospheric } \\
\text { temperature and } \\
\text { humidity fields }\end{array}$ & $\begin{array}{l}\text { mm-wave atmpspheric } \\
\text { sounding/GNSS radio } \\
\text { occultation }\end{array}$ & Comparable/lower & $\begin{array}{l}\text { In development/flight } \\
\text { proven }\end{array}$ & Exploratory/mature \\
\hline $\begin{array}{l}\text { Cloud properties, } \\
\text { liquid qater and } \\
\text { precipitation }\end{array}$ & $\begin{array}{l}\text { mm-wave atmpspheric } \\
\text { sounding }\end{array}$ & Lower & In development & Exploratory \\
\hline $\begin{array}{l}\text { Disaster } \\
\text { monitoring }\end{array}$ & High resolution cameras & Comparable & In development & Mature \\
\hline $\begin{array}{l}\text { Earth radiation } \\
\text { budget }\end{array}$ & Uncooled microbolometers & Comparable & In development & Mature \\
\hline Gravity & Precise accelerometers & Comparable & Flight proven & Mature \\
\hline $\begin{array}{l}\text { Ice sheet } \\
\text { topography }\end{array}$ & $\begin{array}{l}\text { Uncooled minature } \\
\text { spectrometers (VNIR, with } \\
\text { multi-angular } \\
\text { measurements) }\end{array}$ & Marginal & In development & Mature \\
\hline $\begin{array}{l}\text { Land surface } \\
\text { temperature }\end{array}$ & Uncooled microbolometers & Lower & In development & Mature \\
\hline Land topography & GNSS reflectometry & Marginal & In development & Conceptual \\
\hline $\begin{array}{l}\text { Lightning } \\
\text { detection }\end{array}$ & Photodiodes & Comparable & In development & Mature \\
\hline Magnetic field & Vector magnetometers & Lower & Flight proven & Mature \\
\hline Ocean altimetry & GNSS reflectometry & Marginal & In development & Conceptual \\
\hline Ocean color & $\begin{array}{l}\text { Uncooled miniature } \\
\text { spectrometers (UV + VNIR) }\end{array}$ & Lower & In development & Mature \\
\hline $\begin{array}{l}\text { Ocean surface } \\
\text { salinity }\end{array}$ & GNSS reflectometry & Lower & In development & Conceptual \\
\hline
\end{tabular}




\begin{tabular}{|c|c|c|c|c|}
\hline $\begin{array}{l}\text { Ocean surface } \\
\text { temperature }\end{array}$ & Uncooled microbolometers & Lower & In development & Mature \\
\hline $\begin{array}{l}\text { Ocean surface } \\
\text { winds }\end{array}$ & GNSS reflectometry & Lower & In development & Mature \\
\hline Sea ice cover & $\begin{array}{l}\text { mm-wave atmospheric } \\
\text { sounding }\end{array}$ & Lower & In development & Exploratory \\
\hline Snow cover & $\begin{array}{l}\text { Uncooled minature } \\
\text { spectrometers (NIR)/mm- } \\
\text { wave atmosperhic sounding }\end{array}$ & Lower/lower & $\begin{array}{l}\text { In development/in } \\
\text { development }\end{array}$ & Mature/mature \\
\hline Soil moisture & GNSS reflectometry & Lower & In development & Conceptual \\
\hline Vegetation & $\begin{array}{l}\text { Uncooled minature } \\
\text { spectrometers (VNIR) }\end{array}$ & Lower & Flight proven & Mature \\
\hline
\end{tabular}




\section{Appendix B: Current Nanosatellite Database Data [35]}

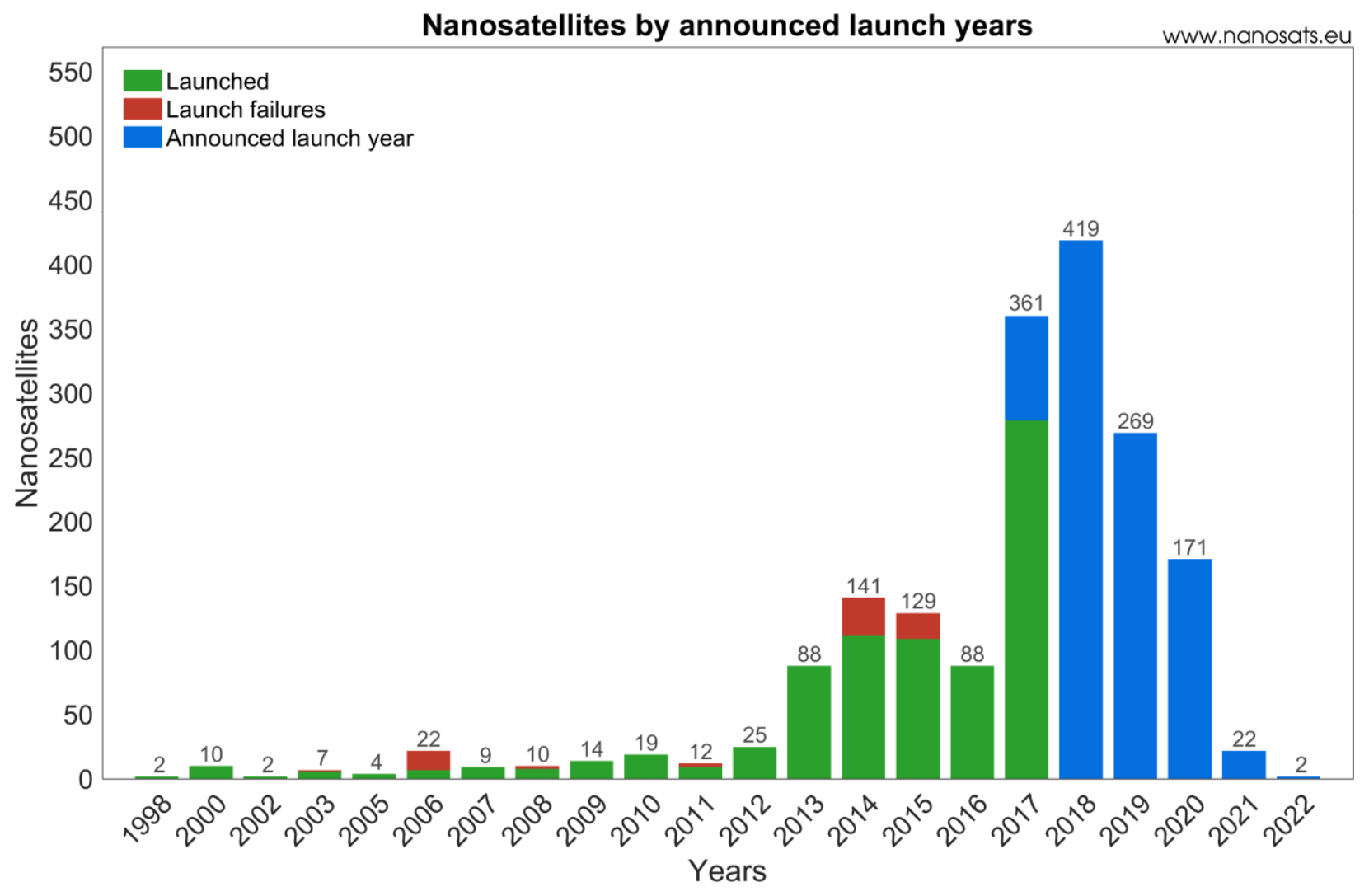




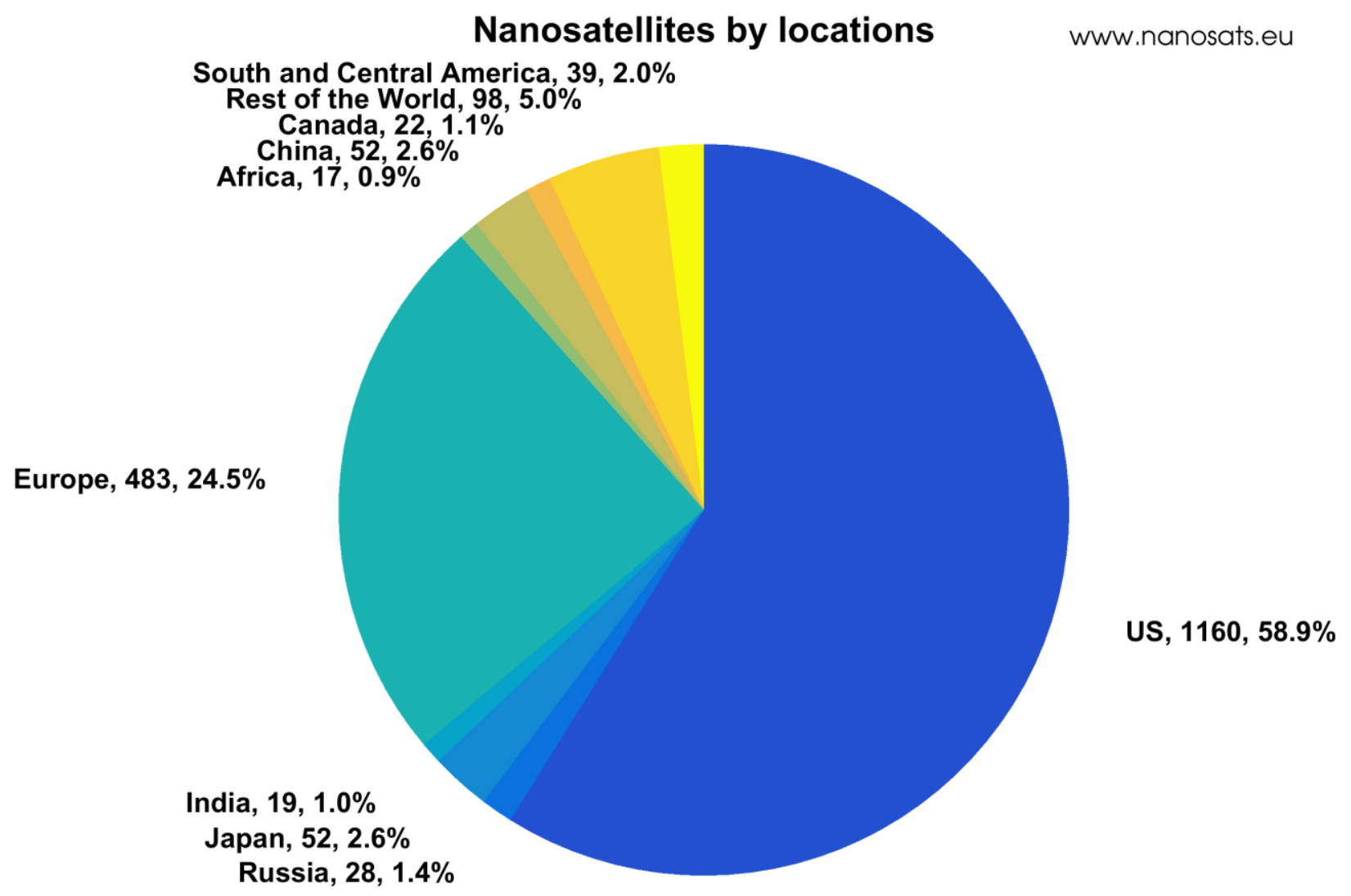




\section{Appendix C: Flight Software}

Program 1: Master CPU Flight Software

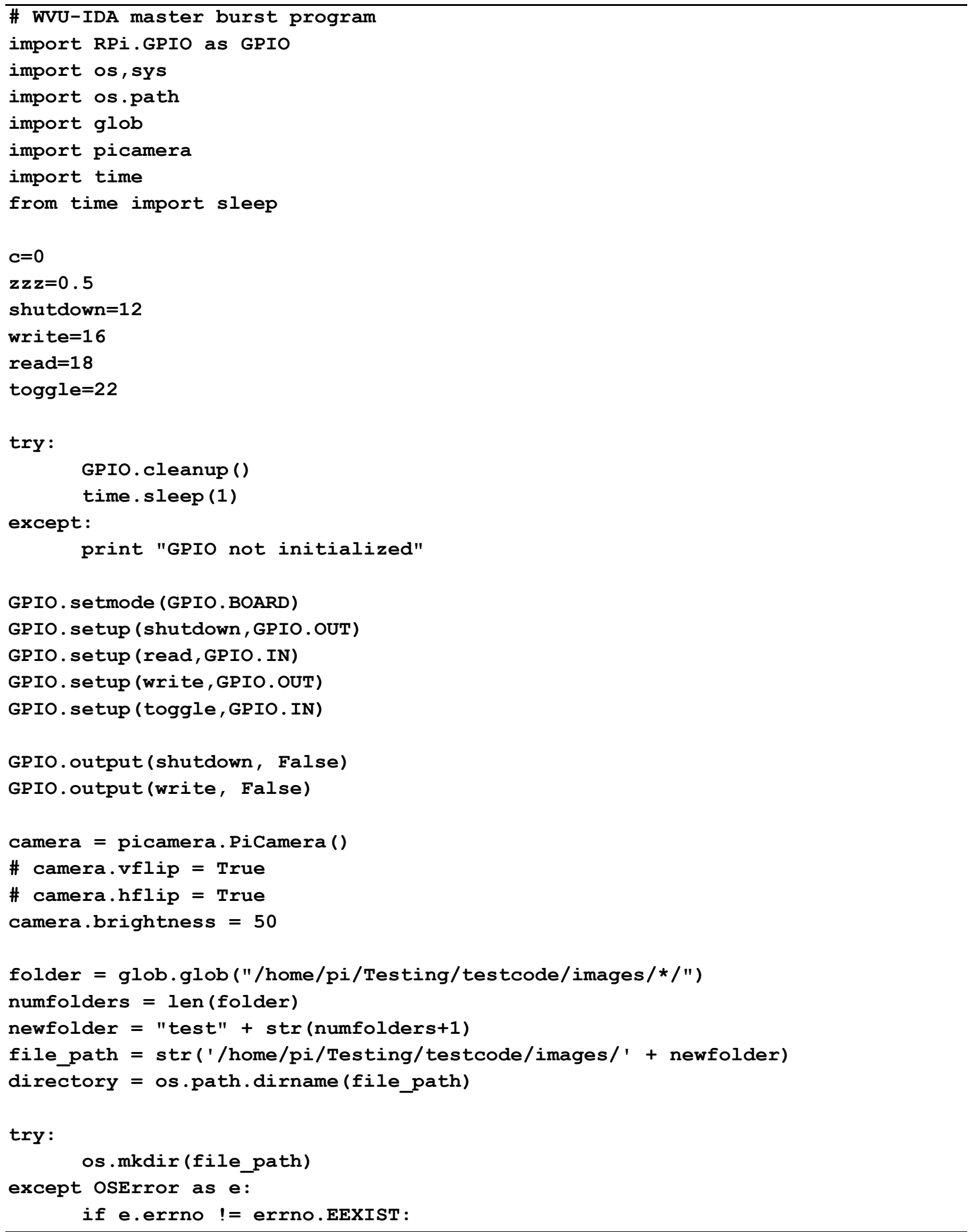




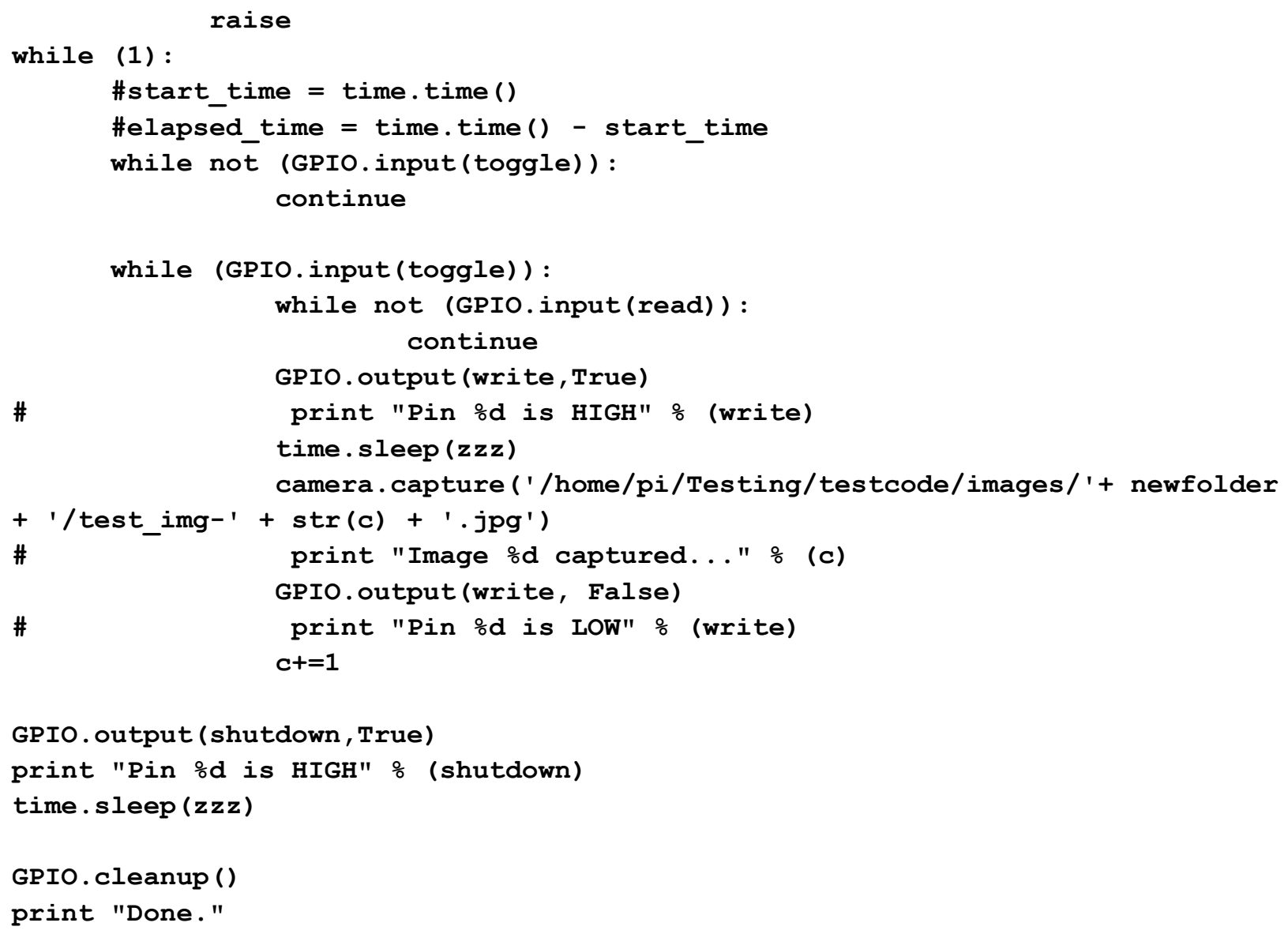


Program 2: Slave CPU Flight Software

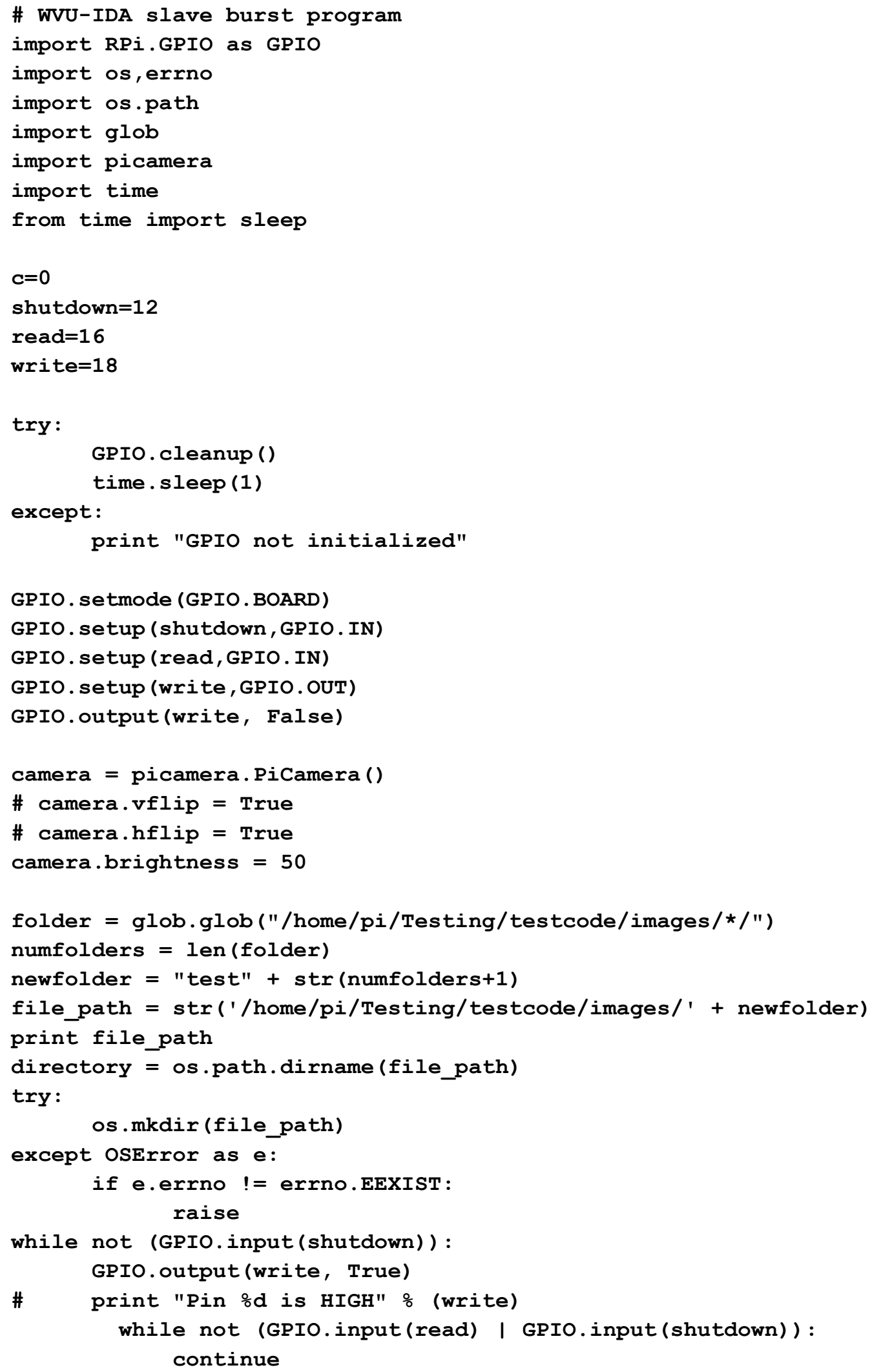




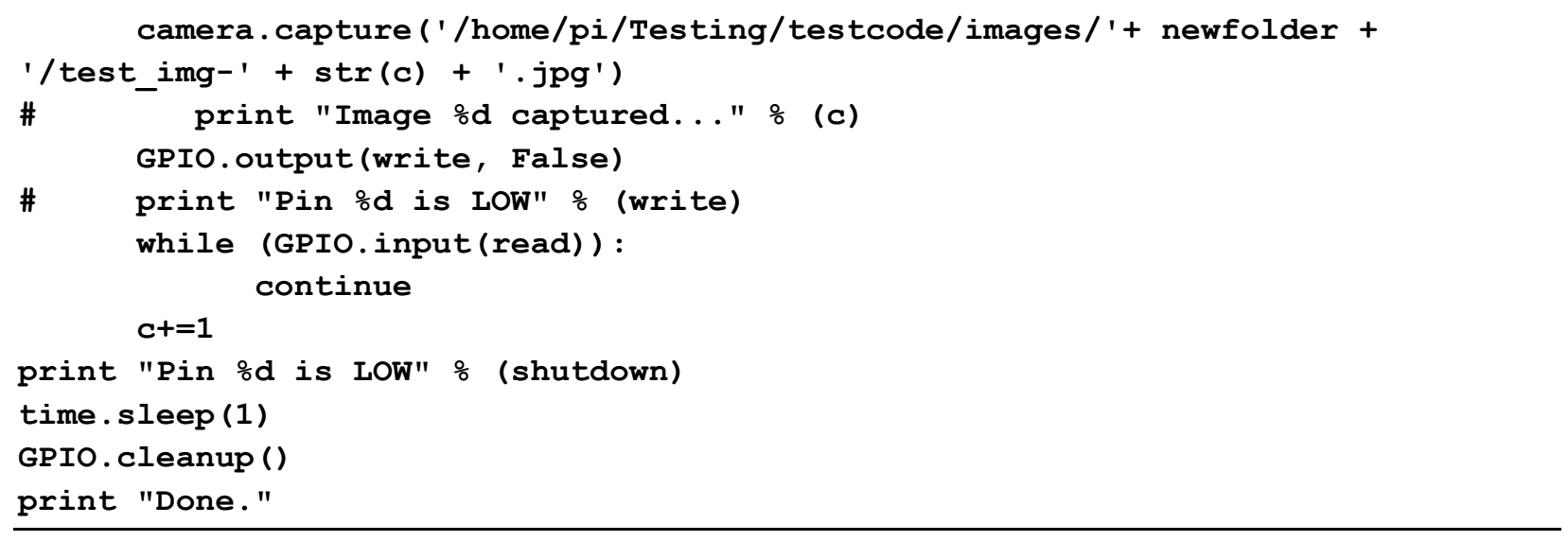




\section{Appendix D: Electronic Parts List}

\begin{tabular}{|l|}
\hline U1: LM2731YMF/NOPB \\
\hline C1: C3216X7R1E225K160AA \\
\hline C2: GRM31CR71A226KE15L \\
\hline Cf: CC1206KRX7R9BB471 \\
\hline D1: MBR0520-TP \\
\hline L1: CDRH125NP-100MC \\
\hline R1: Determined based on desired output voltage - ERJ-8ENF4122V \\
\hline R2: ERJ-8ENF1332V \\
\hline $\begin{array}{l}\text { R3: R3 was replaced by a short (using a solder blob) since ON/OFF control was not } \\
\text { needed. }\end{array}$ \\
\hline
\end{tabular}

